RADC-TR-76-55

Final Technical Report

March 1976

\title{
COAT MEASUREMENTS AND ANALYSIS
}

Hughes Research Laboratories

\author{
Sponsored by \\ Defense Advanced Research Profects Agency \\ ARPA Order 1279
}

Approved for public release;

distribution unlimited.

The views and conclusions contained in this document are those of the authors and should not be interpreted as necessarily representing the official policies, either expressed or implied, of the Defense Advanced Research Projects Agency or the U. S. Government.

\author{
Rome Air Development Center \\ Air Force Systems Command \\ Griffiss Air Force Base, New York 13441
}

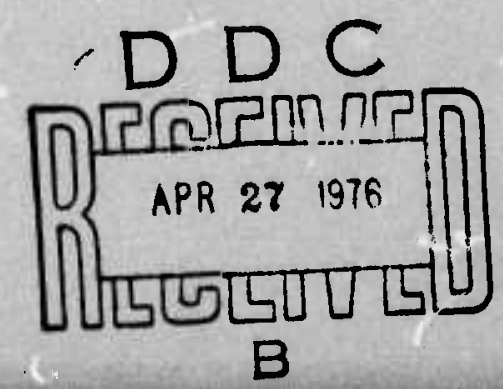



COAT MEASUREMENTS AND ANALYSIS

J. E. Pearson

W. P. Brown, Jr.

A. Kokorowski

M. E. Pedinoff

c. Yeh

\begin{abstract}
Co:tractor: Hughes Aircraft Company
Contract Number: F30602-75-C-0001

Effective Date of Contract: 2 July 1974

Contract Expiration Date: 30 June 1975

Amount of Contract: $\$ 316,766.00$

Program Code Number: 5E20

Period of work covered: Jul 74 - Jul 75

Erincipal Investigator: Mr. J. E. Pearson

Phone: 213 456-6411, ×283

Project Engineer: Robert F. Ogrodnik

Phone: 315 330-4306
\end{abstract}

Approved for public release;

distribution unlimited.

This research was supported by the Defense Advanced

Research Projects Agency of the Department of

Defense and was monitored by Robert F. Ogrodnik

(OCTM), Griffiss AFB NY 13441. 


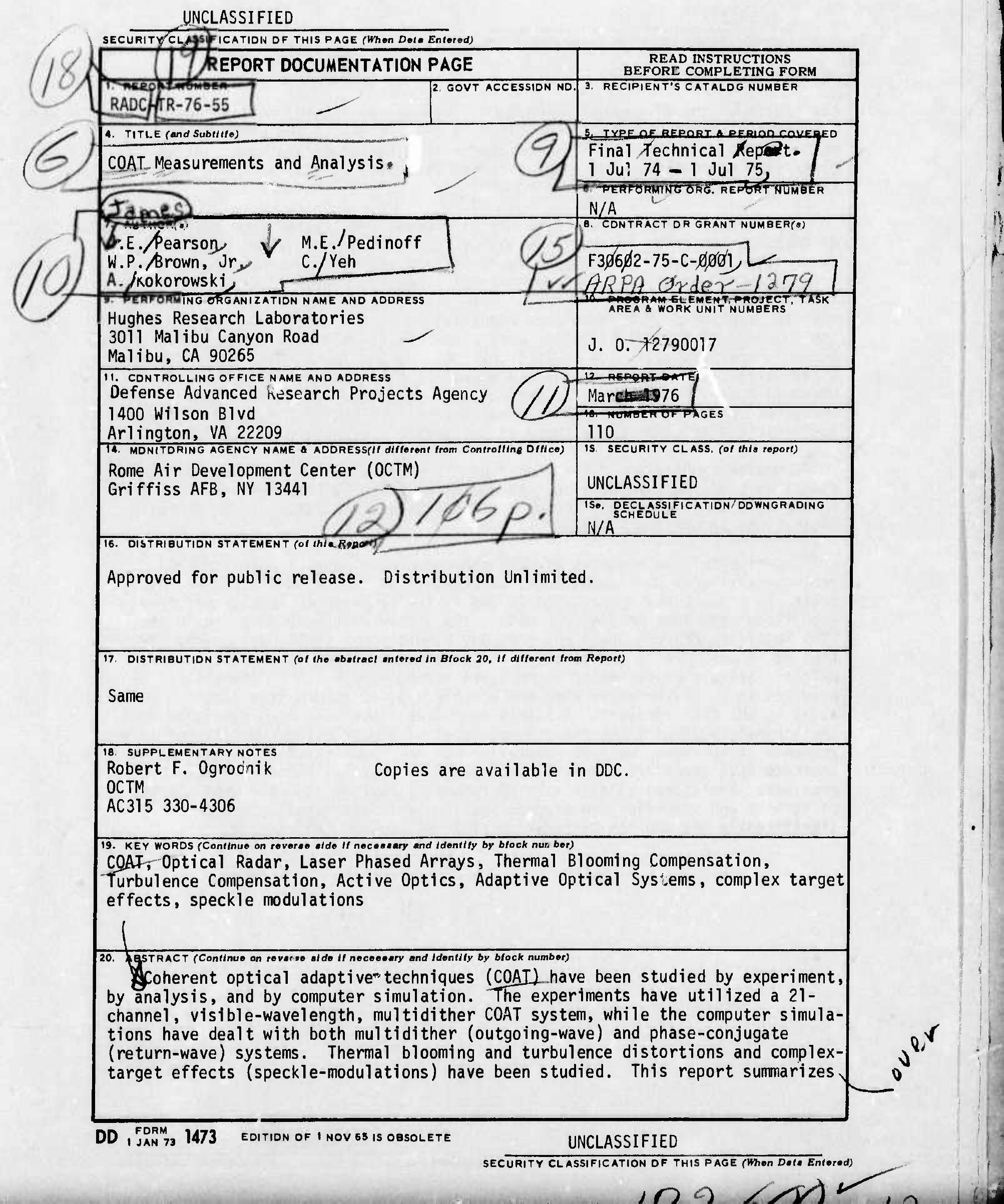


UNCLASSIFIED

SECURITY CLASSIFICATION OF THIS PAGE(Whon Data Eniarrod)

\section{Abstract (continued)}

5

the status of the 21-channel DARPA/RADC, visible-wavelength, multidither COAT experimental model and associated hardware and its use in the experimental measurements on this contract _ Computer simulation studies of propagation distortion compensation and complex target (speckle) effects are also described. Recommendations are made for future work.)

Experimental observations with the 21-channel COAT system show that blooming distortions occurring in the first $30 \%$ of the focused propagation path can be compensated, leading to roughly a factor of 1.5 increase in peak focused-beam ircadiance. Depending on the experimental geometry, howe:ser, correction factors from 1.0 to 4.0 have been observed. Both experimental and computer simulation results have shown that turbulence compensation performance is not degraded by the presence of thermal blooming, even though little blooming compensation may occur. Experimentally, the addition of tracking and focus controls to 18-channe, planar-array, COAT phase control had no effect for blooming distortions, but produced some correction for an artificially-generated turbulence alone. The 18-channel phase controls could remove essentially 100, of the turbulence distortions in most cases where there was no significant wavefront tilt error.

$\Delta$

Computer simulation of phase-colijugate (return-wave) COAT systems has shown almost no blorming compensation. In addition, there is some evidence that a return-wave COAT system may reduge the focal-plane irradiance if the blooming distortions become too strong

$\mathbf{E}_{\mathrm{E}}$ Experimental measurements of COAT operation with equal transmitter and receiver apertures and semidiffuse, extended-glint scotchlight surfaces have failed to produce any degradation in the system convergence level, ever though significant spurious modulations within the dither band were observed in the COAT receiver. KExperimental and computer simulation results have shown, however that it is posstple to reduce the COAT system convergence level if spurious, multiplicative refeiver noise signals are strong enough. Such signals can be produced in a COAX receiver when the speckle pattern return (the target signature) moves by the COAT receiver. A simple analytical model has been developed that can accurately predict the convergence level of a multidither COAT system in the presence of spurious receiver modulation noise. These studies, as well as other , indicate that there are potential problems with speckle-induced noise in a COAT receiver. Additional studies will be required, however, to show what classes of targets and scenarios can produce spurious receiver signals that can significantly degrade the performance of a multidither COAT system. 


\section{PREFACE}

This report was prepared by Hughes Research Laboratories, Malibu, California under Contract F30602-75-C-0001. It describes work performed from 1 April 1975 to 1 June 1975 and summarizes the principal results of the entire contract during the period I July 1974 to 1 July 1975. The principal investigator and principal scientist is Dr. James E. Pearson. The project is part of the adaptive optics program in the Opto-Electronics Department, managed by Dr. Viktor Evtuhov, at the Hughes Research Laboratories. 
LIST OF ILLUSTRATIONS

GLOSSARY OF ABBREVIATIONS AND ACRONYMS . . . . . . . . 13

SUMMARY

INTRODUCTION . . . . . . . . . . . . . 19

A. Program Objectives . . . . . . . . . 19

B. Research Program Plan . . . . . . . . 19

C. Organization of this Report . . . . . . . 21

II COAT SYSTEM HARDWARE . . . . . . . . . . . . . 23

A. Planar Array Phase Controls . . . . . . . 23

B. Multidither Tracking and Focus 26

C. Thermal Blooming Generators . . . . . . 32

D. Turbulence Phase Screen . . . . . . . 36

E. Laboratory Optical Arrangement . . . . . . 38

III EXPERIMENTAL TECHNICAL ACCOMPLISHMENTS . . . . . . . 43

A. Thermal Blooming Compensation . . . . . . 43

B. Turbulence Plus Blooming 60

C. Complex Target Effects . . . . . . . . 64

IV ANALYTICAL TECHNICAL ASSOMPLISHMENTS • • • • • • • • 73

A. Computer Simulation of COAT
Compensation . . . . . . . . . 73

B. Complex Targets and Speckle $8 . . . . . .88$ Modulations 


\section{SECTION}

SUMMARY AND CONCLIUSIONS . . . . . . . . . . . 105

A. Turbutence and Brooming Conpensation . . . . . 105

B. Complex Target Effects Caused by Speckle Modulations . . . . . . . . . 106

C. Recommendations for Additional Investigations ............... 106

REFERENCES ......................... 109 


\section{LIST OF ILLUSTRATIONS}

\section{FIGURE}

1 Revised research program plan showing COAT tasks and scheduling

2 CCAT system block diagram showing additional multidither servo loops for tracking and focus control

RADC/COAT multidither electronics

Beam Active Track (BAT) focus and

tracking controls

Piezo-electrically-driven, variable-

radius spherical mirror used for autofocus control

Measured convergence time of BAT controls

Tracking performance of COAT controls . . . . . . . 31

Static gas absorption cell . . . . . . . . . . 33

9 Flowing gas cell for thermal blooming studies

10 Flowing liquid cell for thermal

blooming studies

11 Phase screen for generating artificial

turbulence effects

$$
\therefore
$$

12 Block diagram of experimental arrangement

used ir all COAT experiments . . . . . . . . . .

13 Optical paths for static liquid cell

blooming experiment

14 Thermal blooming compensation with a

thin, static-liquid blooming cell

located in the near-field of the

transmitter

15 Target irradiance with COAT correction

for various cell locations 
17 Forced convection thermal blooming . . . . . . . . 52

18 Thermal blooming compensation . . . . . . . . 53

19 Thermal blooming compensation data obtained with flowing gas cell . . . . . . . . 56

20 COAT thermal blooming compensation with a flowing liquid medium .. . . . . . . . 56

21 COAT correction with a flowing liquid medium that is in the first $72 \%$ of the propagation path..... . . . . . . . 5

22 Artificial turbulence frequency spectra . . . . . .

COAT colrection for turbulence in the presence f tooming distortions

COAT receiver power spectrum with extended, ming targets . . . . . . . . . .

25 Expe:ime tal set tp to determine effect of spuricis: amplitude modulations in a COAT recenis

Effect of acollsto-optic modulation on DARPA, RALC COR T system as a function of total moduiation bandwidth . . . . . . . . . .

Adaptive optical system simulation flow

Time sequence of events in multipulse adaptive optics simulation . . . . . . . . . .

Streh 1 ratio associated with atmospheric turbulence versus number of mirror actuators

Strehl ratio due to Kolmogorov turbulence . . . . . .

31 Turbulence compensation produced by computer simulation using 37-eiement deformable mirror

32 Turbulence compensation produced by computer simulation using 37-element deforrable mirror 


\section{FIGURE}

33 Phase conjugate COAT correction for thermal blooming at $10.6 \mu \mathrm{ml}$ for a truncated gaussian beam with beam slewing . . . . . . . . . . .

34 Phase conjugate COAT correction for thermal blooming and turbulence at $3.8 \mu \mathrm{m}$ for a truncated gaussian beam with beam slewing . . . . . .

35 Phaser diagram of a partially converged, Nelement COAT array defining the quantities $\alpha$ and $\alpha_{0}$ used in the text . . . . . . . . . . .

Statistical expectation value of the mean convergence level, $I_{M}$, for an 18-channel multiditner COAT system

37 Theoretical dither power and speckle power versus convergence level for an 18-element

COAT servo simulation showing addition of "speckle" noise generator .. . . . . . . . . .

COAT convergence levels as a function of speckle noise amplitude as observed by computer simulation

Convergence level versus a $a_{\text {max }}$ theory versus experiment for an 18-êlemerit COAT array 
GLOSSARY OF ABBREVLATIONS ANI) ACRONYMS

1. COAT: Coherent Optical Adaptive Techniques. Any one of a number of adaptive optical techniques. Also used as a

2. BAR: Beam Active Track. Name given to the multidither tracking and focus controls built and studied on this
contract.

3. HRL: Hughes Research Laboratories, Malibu, California

4. GRC: General Research Corporation, McLean, Virginia

5. RADC: Rome Air Development Center, Rome, New York

6. NSWC: Naval Surface Weapons Center, Silver Spring, Maryland

7. DARPA: Defense Advanced Research Frojects Agency,
Washington, D.C. 
This research program on coherent optical adaptive techniques (COAT) has three pirmary objectives: (1) to experimentally study the ability of multidither COAT system to compensate for thermal blooming and turbulence, particularly when it operates against complex, multipleglint targets; (2) to use computer simulations to cross-check the experimental results and to investigate areas beyond the capabilities of the experimental hardware; (3) to perform preliminary studies of the effects of speckle noise modulation on COAT system convergence and performance. This report is the final contract report and reviews the experimental, analytical, and computer simulation work performed during the 12 -month contract period from 1 July 1974 to 1 July 1975.

The experimental studies employed the 18 -channel multidither COAT planar array, which operates at $0.488 \mu \mathrm{m}$ and was constructed on a previous program. This system and other related hardware is discussed in Section II of the report. Thermal blooming compensation measurements were made using this COAT system with four different absorption cells: two liquid and two gas. The two gas cells, each $50 \mathrm{~cm}$ long, were constructed during this contract. The liquid cells were part of the Hughes IR\&D program. Each cell was used in a slightly different beam geometry as discussed in Section III, but each geometry can be scaled to an interesting $10.6 \mu \mathrm{m}$ scenario. The ratio of the peak target irradiance with COAT correction to that without was observed to be $1.0,1.3,1.5$, or 4.0 depend ing on the experimental geometry. No differences were observed between blooming obtained with liquid or gaseous media. The greatest correction is observed when the blooming distortions are in the first $30 \%$ of the focused propagation path; the least correction occurs when the blooming is confined to the last 50 to $60 \%$ of path. In all cases, the COAT system convergence level was stable, with no evidence of oscillation or instability as the blooming distortions were increased well beyond that present with the optimum transmitter power. 
Multidither adaptive tracking and foc s controls were designed and constructed to be used with the 18 -channel COAT system. The design goals of a $100 \mathrm{~Hz}$ tracking sy $\mathrm{em}$ and a $10 \mathrm{~Hz}$ focusing system were met by the hardware. In the thermal blooming measurements, these controls produced no further increase in peak target ir radiance over that provided by the 18-channel planar array; in one turbulence-only experiment, however, sorie correction was observed.

We have experimentally studied the effects of target motion-induced speckle modulation on the 18-channel COAT system. A scaled laboratory experiment using a rotating scotchlite target produced a wideband speckle noise spectrum over the dither frequency band which did not observably interfere with the convergence properties of the system. In another series of experiments, an acousto-optic mociulator with $64 \%$ modulation efficiency was placed in the DARPA/RADC COAT optical system and swept over a variable frequency bandwidth in a time comparable to the servo loop convergence time. The speckle modulation artificially generated by this technique produced interference signals over the dither band greater than the normal dither signals, and produced converged power degradation of $35 \%$.

Computer simulation modeling of COAT system performance in the presence of speckle noise modulation has been used to obtain the average convergence level of an 18-channel multidither COAT system in the presence of this noise. An oscillation of the system about the average convergenc? levei has been observed. A statistical analysis of the COAT system and of the speckle noise has been developed for use in explaining the simulation results. Good agreement has been observed between the analytical model and the computer simulation data. The experimental and theoretical results obtained thus far indicate that the system performance can be significantly degraded if the modulation is large enough, but the systematic evaluation of which targets, target ranges, and target motions, if any, can actually produce such large modulations are being pursued on other programs. To date, however, our experimental results with the 18-channel COAT system (equal transmitter and receiver apertures) and realistic targets indicate that speckle modulations will not seriously degrade COA.T system performance. This is a significant conclusion since any operational 
COAT system wili deal with semidiffuse, multiple glint targets that do produce speckle-like target signatures.

Turbulence compensation in the presence of thermal blooming has been studied by experiment and by computer simulation. We have observed that turbulence compensation is not significantly degraded by the existence of thermal blooming, even though the thermal blooming distortions may not be removed by the COAT s, stem. This result is particularly significant for laser systems operating at $3.8 \mu \mathrm{m}$ or at $10.6 \mu \mathrm{m}$ with larger $(>1 \mathrm{~m})$ apertures where turbulence distortions dominate the beam degradation. Just as blooming and turbulence can be treated separately and later combined to determine the total beam distortion, so can the COAT compensation for each type of distortion be treated separately. In the experimental measurements, identical compensation performance was observed with single glint targets and with semidiffuse, rough-surface, rotating spherical targets.

Our computer simulation studies of COAT compensation for thermal blooming have been restricted to return-wave (e.g., phase-conjugate) COAT systems because of the large computational cost when the multidither servo code is combined with the nonlinear propagation code. This point is discussed further in Section IV. The operation of return-wave systems is significantly different with nonlinear distortions than that of a multidither, outgoing-wave system (like the 18-charnel experimental system). We have observed very little blooming compensation in the simulations and have found that return-wave systems can actually reduce the target irradiance when the transmitted power exceeds the optimum power (that power which produces maximum target irradiance). We suspect that this type of behavior may be characteristic of any return-wave system, including those that employ compensated imaging concepts (imaging-COAT systems).

Based on previous data* and on the investigations summarized in this report, we conclude that a multidither, outgoing-wave COAT system can remove 70 to $100 \%$ of turbulence-induced beam distortions, $100 \%$ of fixed beam distortions (optics, etc.), can provide about a factor of 1.5 to 2.0 improvement in peak beam irradiance in the presence of thermal

*J. E. Pearson, "Coherent Optical Adaptive Techniques," Contract F30602. 73-C-0248m Final Technical Report, RADC-TR-75-46, Jan. 1975. 
blooming, and can accomplish all of the se functions simultaneously, limited only by the number of correction channels and by the system speed of response. The compensation can be achieved with realistic targets having multiple glints, or semidiffuse surfaces. Propagation of high energy laser beams through the atmosphere to produce large focal-plane ir radiances should thus not be limited by the atmosphere if suitable COAT system designs are used and if severe thermal blooming distortions can be avoided. Additional work is indicated in several areas: (1) speckle modulation effects to determine what targets and scenarios can produce speckle modulations that are strong enough to degrade multidither COAT performance and to design COAT systems that are insensitive to such modulations; (2) thermal blooming compensation - to investigate techniques that can minimize thermal blooming distortions; (3) computer simulation - to develop cudes that can simulate thermal blooming compensation with multidither COAT systems and realistic, multiple-glint targets; (4) COAT operation at infrared wavelengths - to demonstrate the hardware technology required for COAT systems at longer wavelengths and higher powers and to investigate target effects (speckle, glint destruction) with realistic target surfaces and geometries; (5) new COAT concepts - to investigate experimentally new servo and system concepts that offer improved compensation performance, greater versatility, greater simplicity in hardware design and implementation and more reliable operation under a wider variety of operational conditions. 
I. INTRODUCTION

A. Program Objectives

There are two primary objectives of this program. The first shjective is to determine the performance limits of multidither coherent optical adaptive techniques (COAT) by performing scaled laboratory experiments which are designed to produce quantitative data on the nature of thermal blooming and turbulence and on the ability of multidither COAT to correct for these distortions, particularly when the target has many moving, time-varying glints. The second objective is to use computer simulation as a cross-check on the experiments and as an analytical tool to extend the understanding of propagation distortions and of COAT systems beyond the capabilities of the experimental hardware.

B. Research Program Plan

The performance of multidither COAT has already been proved with an 18-element visible wavelength system developed and tested on DARPA/ RADC Contract F30602-73-C-0248 which was concluded in July 1974. This same system is used for the laboratory experiments in this program. The computer simulation codes for atmospheric turbulence, thermal blooming, and the COAT system were developed on DARPA/NOL Contract N6092174-C-0249. The previous DARPA/RADC COAT contract also supplied a design for a gas absorption cell which has been used to simulate convectiondominated thermal blooming in the atmosphere.

The research program for this contract, illustrated in Fig. 1, runs from 1 July 1974 through 30 June 1975. The required oral presentation was made on 3 December 1974 as part of the DARPA/NOL Adaptive Optics Symposium held at Lincoln Laboratories. ' A contract arnendment negotiated during the second contract quarter provided for the addition of auto-tracking and autofocus controls to both the DARPA/RADC COAT hardware and the Naval Ordnance Laboratories computer code. A second amendment, negotiated during the third quarter, eliminated a high-power design guidelines task so that time and funds could be directed toward experiments on COAT/ target-signature interactive effects. The schedule in Fig. 1 reflects these changes. 


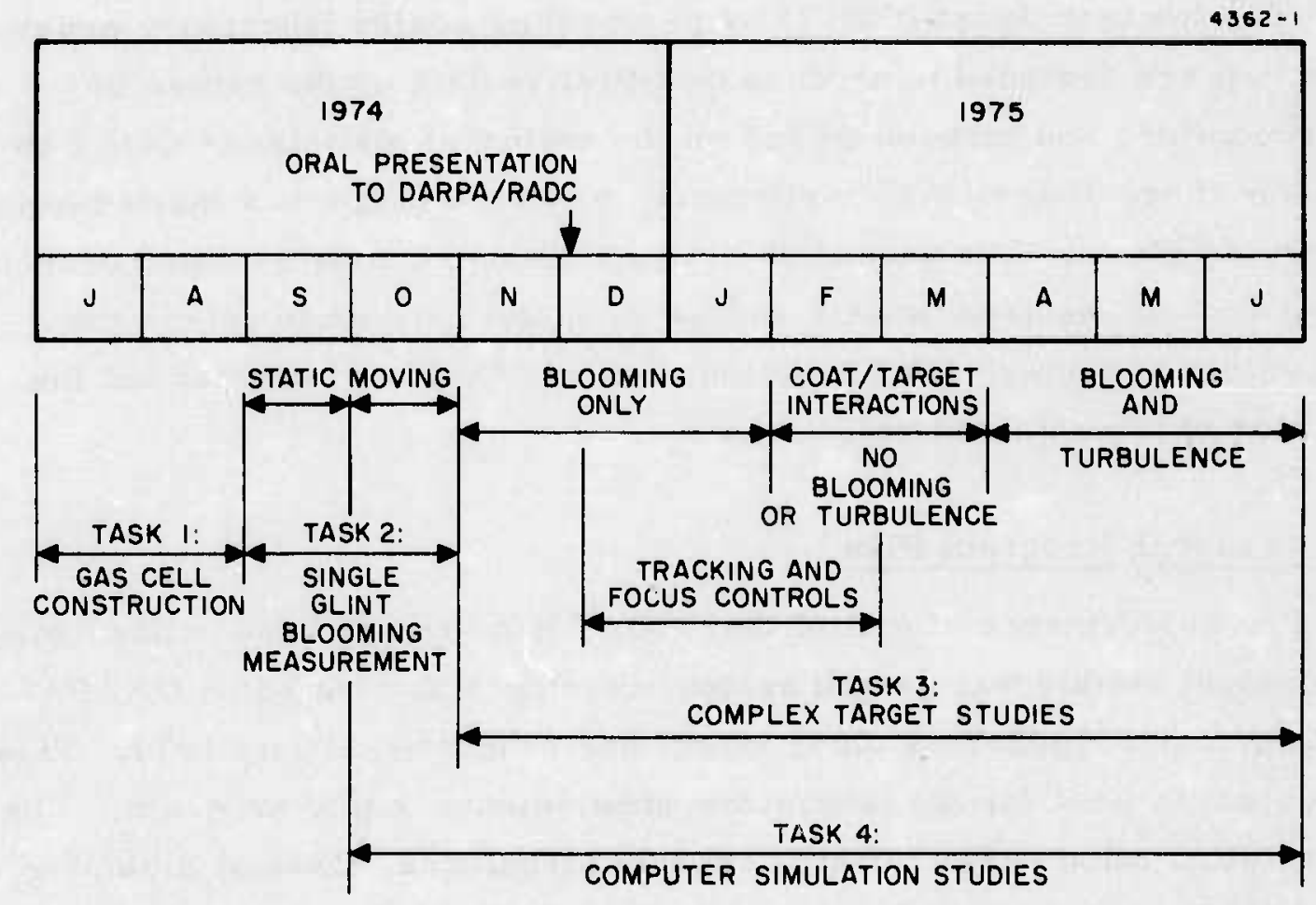

Fig. 1. Revised research program plan showing COAT tasks and scheduling. 


\section{Organization of This Report}

This report serves two functions: (1) it presents the accomplishments of the fourth contract quarter (1 April 1975 to I July 1975); (2) it summarizes the principal accomplishments of the entire contract. The report is thus both the fourth quarterly technical report and the final contract report.

Section II reviews the status of the experimental hardware that has come to be known as the DARPA/RADC COAT system. Items summarized include the 18-channel planar COAT array, the 3-channel Beam Active Track (BAT) system, the associated optical systems used in experimental laboratory studies, and the laboratory distortion generators used to produce thermal blooming and turbulence.

Section III summarizes the experimental results obtained on turbulence and thermal blooming compensation. Preliminary investigations on COAT system operation with complex, extended-glint targets a re presented. The section briefly discusses the effects of spurious receiver modulations produced by moving speckle-patterns (the target signature).

Section IV summarizes the results of the analytical efforts directed toward investigating turbulence and thermal blooming compensation as well as complex target effects. The data presentsd were obtained primarily by computer simulation of the propagation distortions and the COAT system (either phase conjugate or multidither).

Section $V$ summarizes the principal results of the contract and the conclusions that can be drawn from this work. The implications for additional research that should follow this work are presented. 
II. COAT SYSTEM HARDWARE

\section{A. Planar Array Phase Controls}

The original DARPA/RADC COAT system was developed on Contract F30602-73-C-0248. A detailed description of the system is presented in the five contract reports ${ }^{1-5}$ for that program. A block diagram of the complete system is shown in Fig. 2 and Fig. 3 is a photograph of the signal conditioning and servo electronics for the 18-phase control channels. The features and performance of the system are summarized in Tables 1 and 2. As can be seen from Table 2, all of the fundamental multidither COAT concepts have been successfully demonstrated with this system. The remaining issues are no longer concerned with whether or not the system will work, but rather with how well it will work under various conditions. The data obtained on this contract answer the latter question for some cases and succeeding programs now in progress are designed to further quantify the degree of successful COAT compensation for turbulence and blooming with realistic targets.

\section{Table 1. DARPA/RADC Planar Array COAT System Features}

$1.8 \mathrm{~W}, 0.488 \mu \mathrm{m}$, argon laser

Planar output arrays with any configuration containing up to 18 phasecontrolled elements and one reference element.

PZT cylinders and bimorphs used as phase dither and phase correction drivers ("piston" elements). Up to $60^{\circ}$ peak dither modulation and over $\pm 2 \lambda$ correction available at $\lambda=0.488 \mu \mathrm{m}$.

Dither frequencies adjustable from 8 to $32 \mathrm{kHz} ; 1$ channel/frequency or sine/cosine operation.

Chopper-stabilized AGC with $46 \mathrm{~dB}$ dynamic range and $1 \mathrm{msec}$ response time.

Sample-and-hold, slew-offset capability - electronic and mechanical.

Two photomultiplier detectors available: one for "local loop" operation (no path distortion), one for "target loop" operation (path contains turbulence and blooming). 


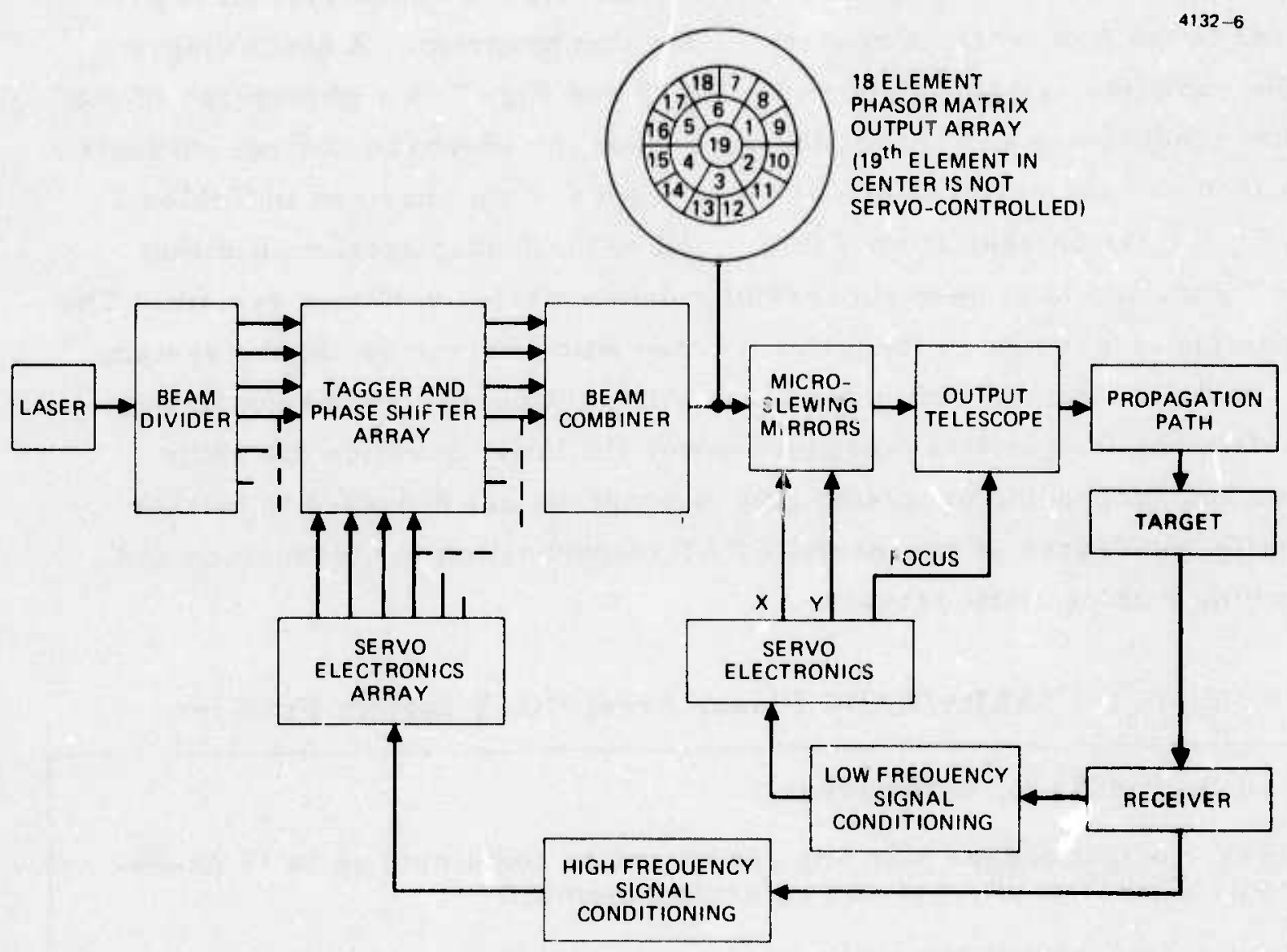

Fig. 2. COAT system block diagram showing additional multidither servo loops for tracking and focus control. 


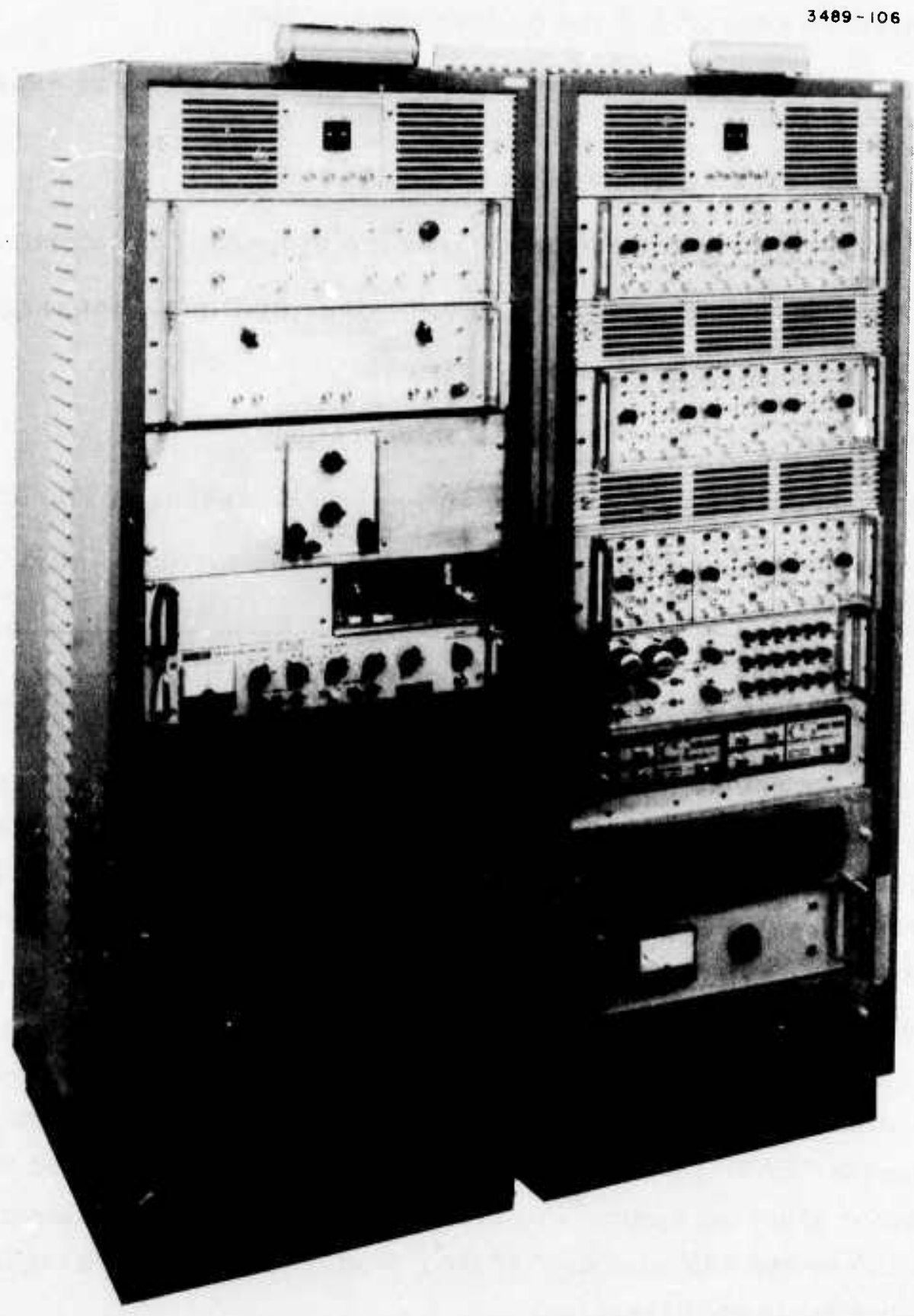

Fig. 3. RADC/COAT multidither electronics: 18-phasecontrol servo channels are shown; the 2 tracking ( $x$ and $y$ ) channels and 1 focus channel are shown in Fig. 4 . 
Table 2. DARPA/RADC Planar Array Performance

Convergence time of $1.5 \mathrm{~ms}$ or less $(10 \%$ to $90 \%)$.

Good agreement between computer simulation of servo and experimental results.

Single glint tracking at rates up to $30 \mathrm{mrad} / \mathrm{sec}$.

$1.3 \mathrm{x}$ diffraction-limited performance (no propagation distortions).

Convergence on strongest glint in a moving, multiple glint target.

Demonstrated turbulence compensation.

Demonstrated thermal blooming compensation.

Demonstrated turbulence compensation in the presence of blooming.

Demonstrated compensation performance with "extended-glint" targets.

B. Multidither Tracking and Focus Controls

Amendment No. 1 to the original contract provided supplemental funds to add three additional multidither control channels to the 18-channel planar array controls: 2 tracking controls and 1 spherical-focus control. Although we usually will refer to the entire COAT system as the "2l-channel DARPA/RADC COAT system," we will have occasion to refer to just the focus and/or tracking controls. In this report, we will use the term "18-channel" to refer to the 18-element pianar array system which produces piston-type phase control on 18 separate transmitted beam segments. The acronym "BAT" (for Beam Active Tracking) will be applied to the focus and/or tracking controls alone. When sp aking of combinations, we will use the terms "20-channel" (COAT plus tracking), or "21-channel" (COAT plus focus plus tracking).

The tracking and focus controls are shown in Fig. 4 and Fig. 5 is a schematic of the focus actuator. The servo control channels were built by dividing the servo parameters of the 18 -channel system by 10 for the tracking channels and by 100 for the focus control. This procedure 


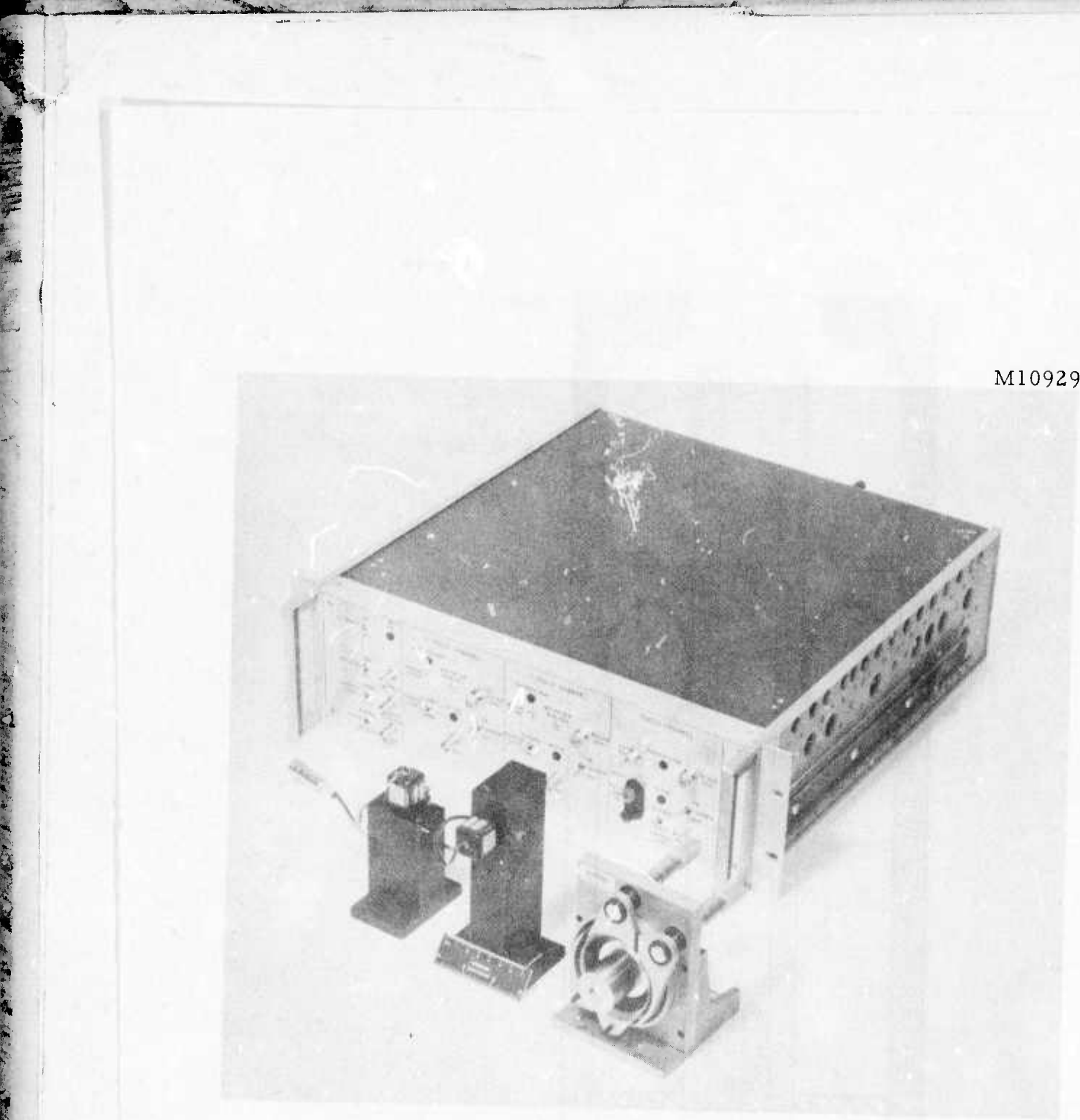

Fig. 4. Beam Active Track (BAT) focus and tracking controls. 


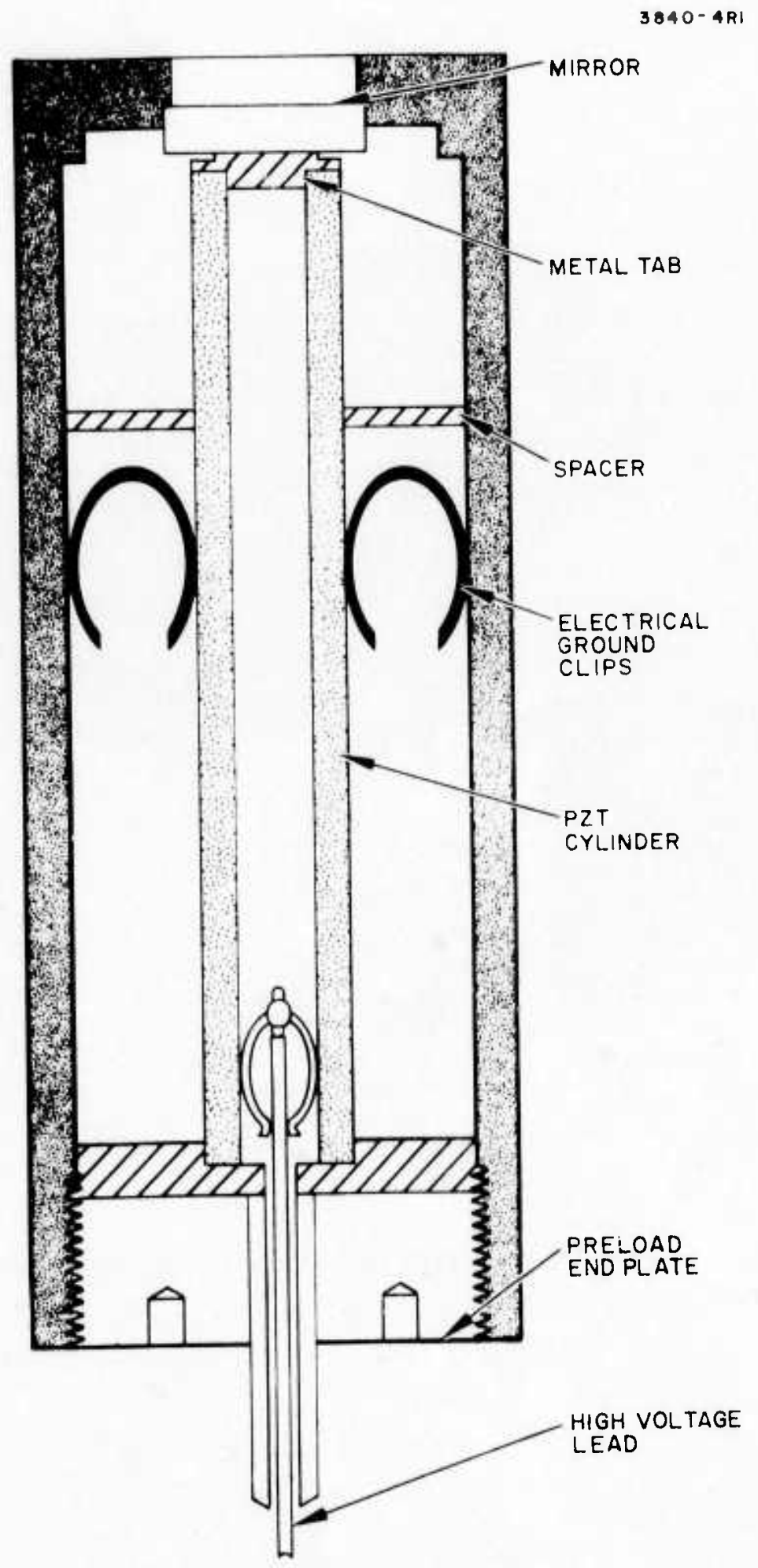

Fig. 5 .

Piezo-electrically-driven, va riable-radius

spherical mirror used for autofocus control. 
was expected to prociuce a tracking servo with a $1 \mathrm{kHz}$ dither and a $10 \mathrm{msec}$ convergence time (100 Hz response) and a focus servo with a $100 \mathrm{~Hz}$ dither and a $100 \mathrm{msec}$ convergence time (10 Hz response). The oscilloscope traces of target irradiance versus time shown in Fig. 6 demonstrate the achievement of these design goals.

Both controls could be made to operate faster since the lowest resonance frequency of the focus actuator is $3.4 \mathrm{kHz}$ and the tracking galvanometer-plus-mirror resonance is at $1.7 \mathrm{kHz}$. Although we chose to op arate the two tracking controls with two separate dither frequencies $(750 \mathrm{~Hz}$ and $1 \mathrm{kHz}$ ), sine-cosine operation will work equally well (producing a conical-scan tracker).

The tracking performance of the controls is shown in Fig. 7. An interesting behavior is observed: the controls do not automatically steer the beam boresight axis onto the glint when the pilase and BAT controls are used together. The stable lock position depends on the initial conditions as shown in the figures. This behavior is not entirely reproducible and is a consequence of the sidelobe structure of the segmented transmitting aperture and of the choice of dither frequencies. (The behavior would not exist if the tracking servo were faster than the phase control servo.) This explanation is discussed in greater detail in the third contract quarterly report. 6

Although the focus control servo has performed as precicted by the design, the actuator shown in Fig. 5 has one problem: it in:-oduces a small, but not insignificant amount of aberration into the optical beam. When the actuator is used with the 13-channel COAT system, the distortion is no problem since the COAT system can completely remove it. The dis tortion does not allow the actuator to be used by itself, however, without introducing undesired beam distortions. Consequently, all of the experiments discussed in Section III that involve the focus control also use the 18-channel COAT system.

The BAT tracking and focus controls have also been implemented into a multidither computer simulation that uses a deformabie mirror as a dither and corrector element. The nonboresighting effects seen in the experiment were thus not observed. We also decided to implement a 

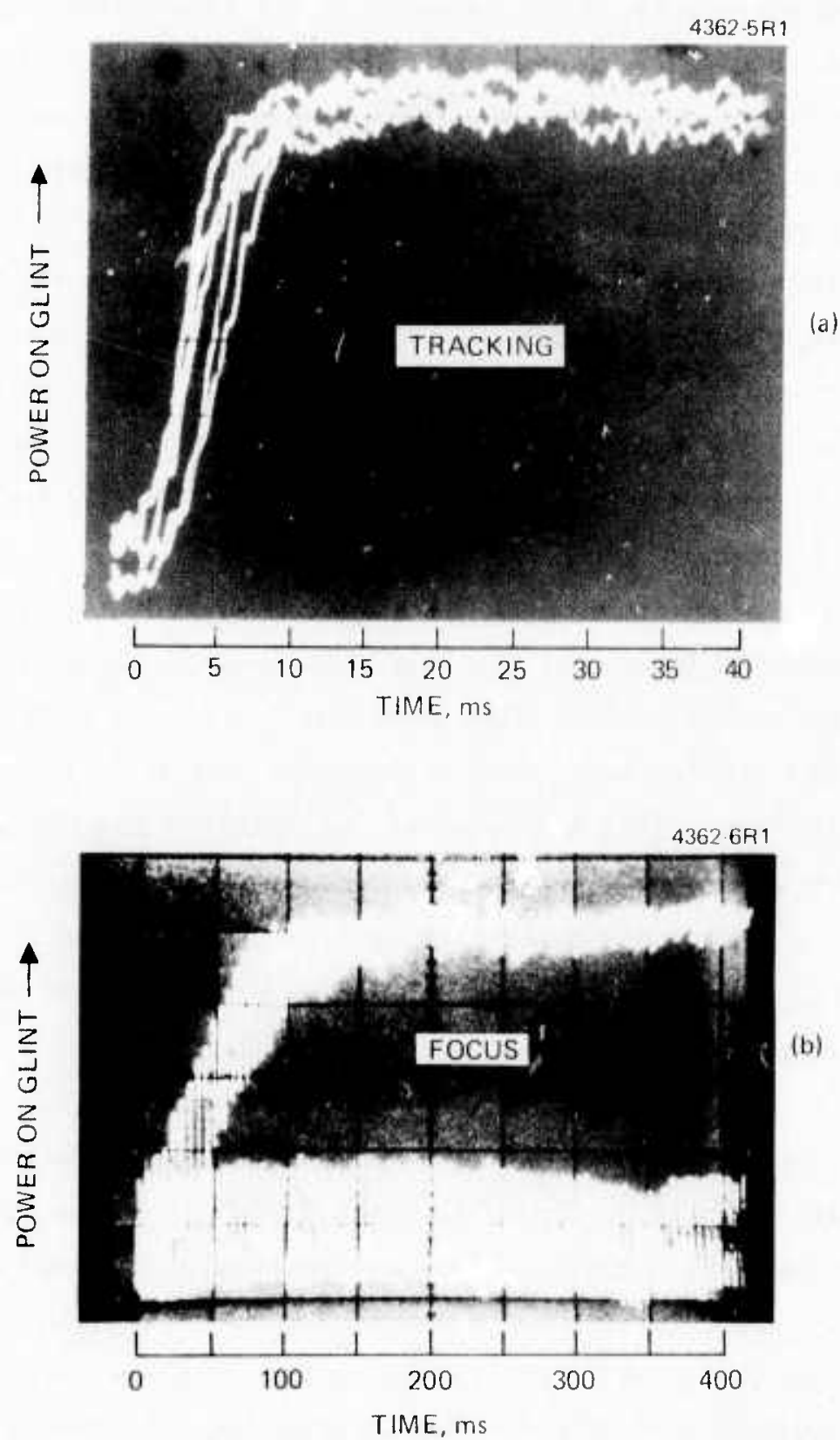

Fig. 6. Measured convergence time of BAT controls. (a) Tracking controls

(b) Focus control. 

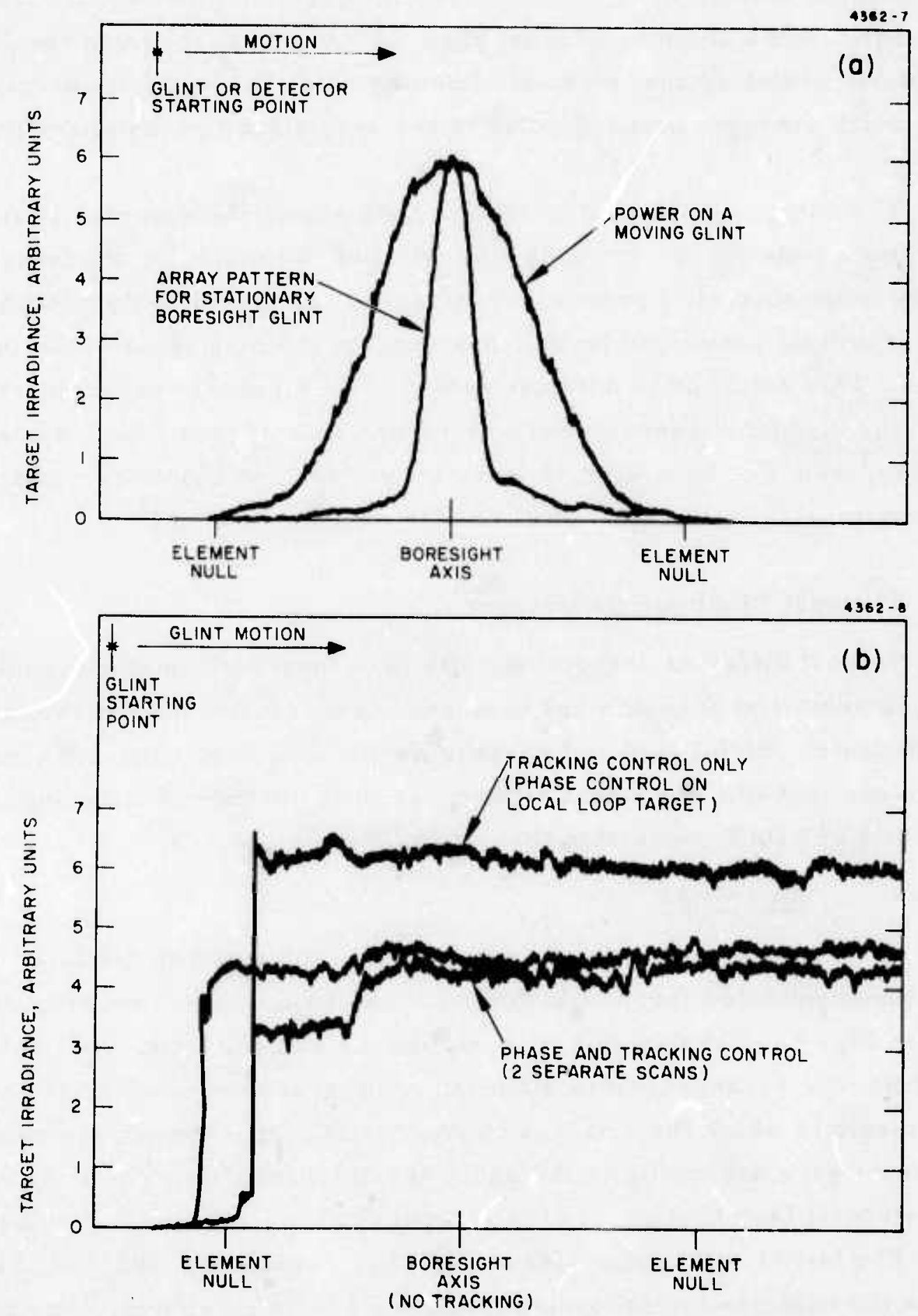

Fig. 7.

Tracking performance of COAT controls. (a) Tracking control off. The formed array pattern is also shown for reference.

(b) Tracking control performance. Curves with tracking control only and with both phase and tracking controls are shown. The ability of the system to lock in a nonoptimum, off-boresight condition is evident. 
two-axis focus control in the simulation, in effect using two cylindrical lens controls that focus along orthogonal axes. This type of focusing should be particularly useful against thermal blooming since the negative thermal lens is much stronger perpendicular to the wind direction than parallel with it.

The computer simulation results have shown the expected $10 \mathrm{msec}$ convergence time for the tracking controls and $100 \mathrm{msec}$ for the focus. With the focus control, however, we observed another initially unexpected result: the final converged level can depend on the magnitude of the initial defocus. This behavior is now understood to be a generalized manifestation

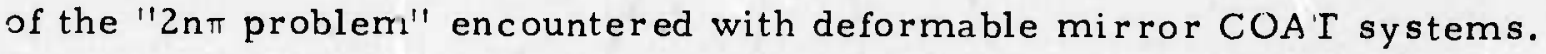
This sinulation has been used to study turbulence and blooming compensation; results of these calculations are discussed in Section IV.

\section{Thermal Blooming Generators}

Several different absorption cells have been built during the course of this contract and each one has been used in a variety of experimental arrangements. Both liquid and gaseous media have been used. We did not observe any medium-dependent differences in the nature of blooming or the amount of COAT correction that could be produced.

1. Gas Cells

Several designs were evaluated ${ }^{5}$ for a cell to produce convection-dominated thermal blooming. The first design ${ }^{7}$ we wmployed is shown in Fig. 8. The design uses a stationary gas cell (Fig. 8(a)) and a moving mirror arrangement to simulate a transverse wind (Fig. $8(\mathrm{~b})$ ). Experiments in which the cell was mounted vertically to minimize convection effects gave substantially the same results as those in which the cell was horizontal (see Section III of this report).

The initial cell design ${ }^{5}$ identified $\mathrm{SF}_{6}$, seeded with $\mathrm{NO}_{2}$ for absorption, as the best gas for producing maximum blooming effects. For reasons that are still not apparent, $\mathrm{SF}_{6}$ did not produce the expected amount of blooming. We theorize that the large number of $V-T$ states in $S F_{6}$ prevents rapid transfer of the energy absorbed by the $\mathrm{NO}_{2}$ into thermal 


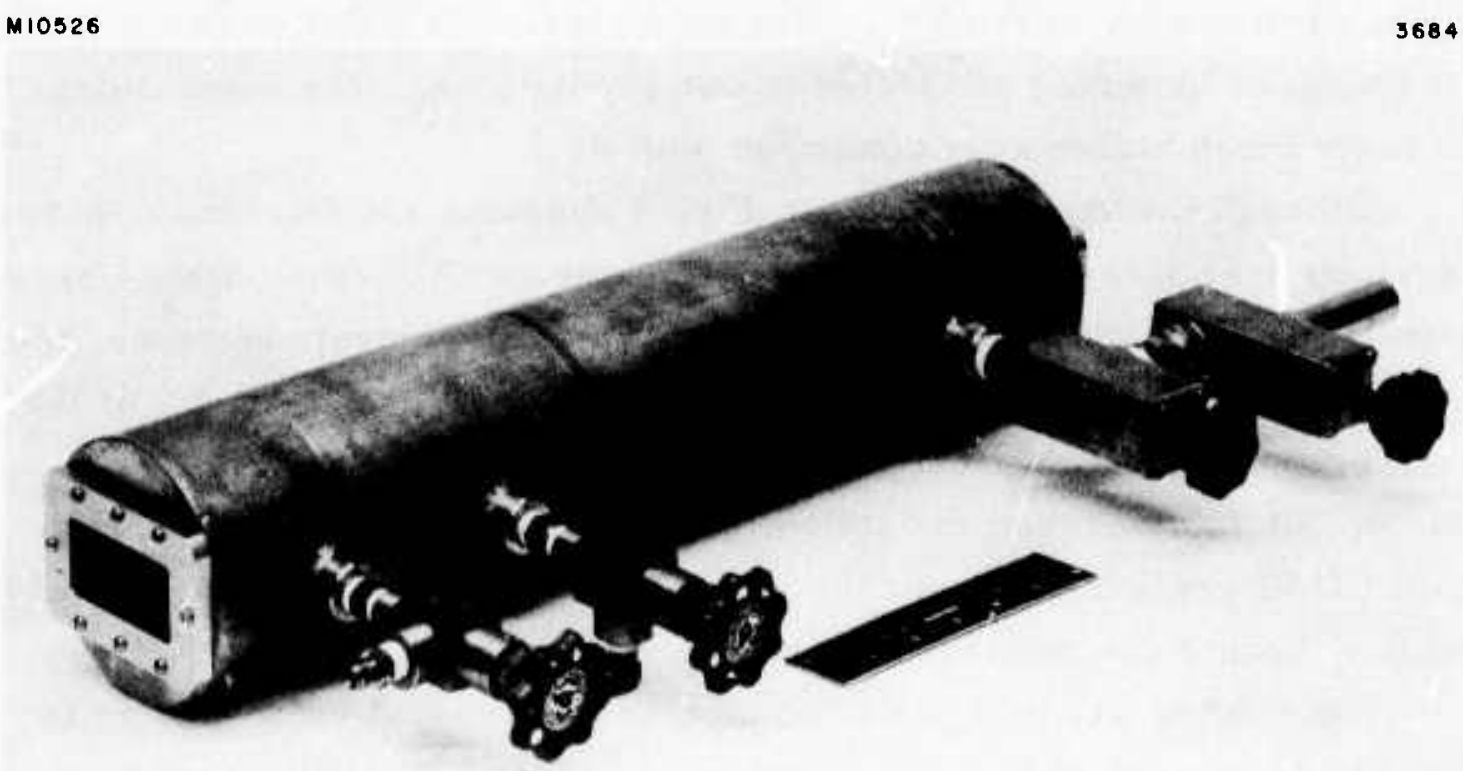

(a)

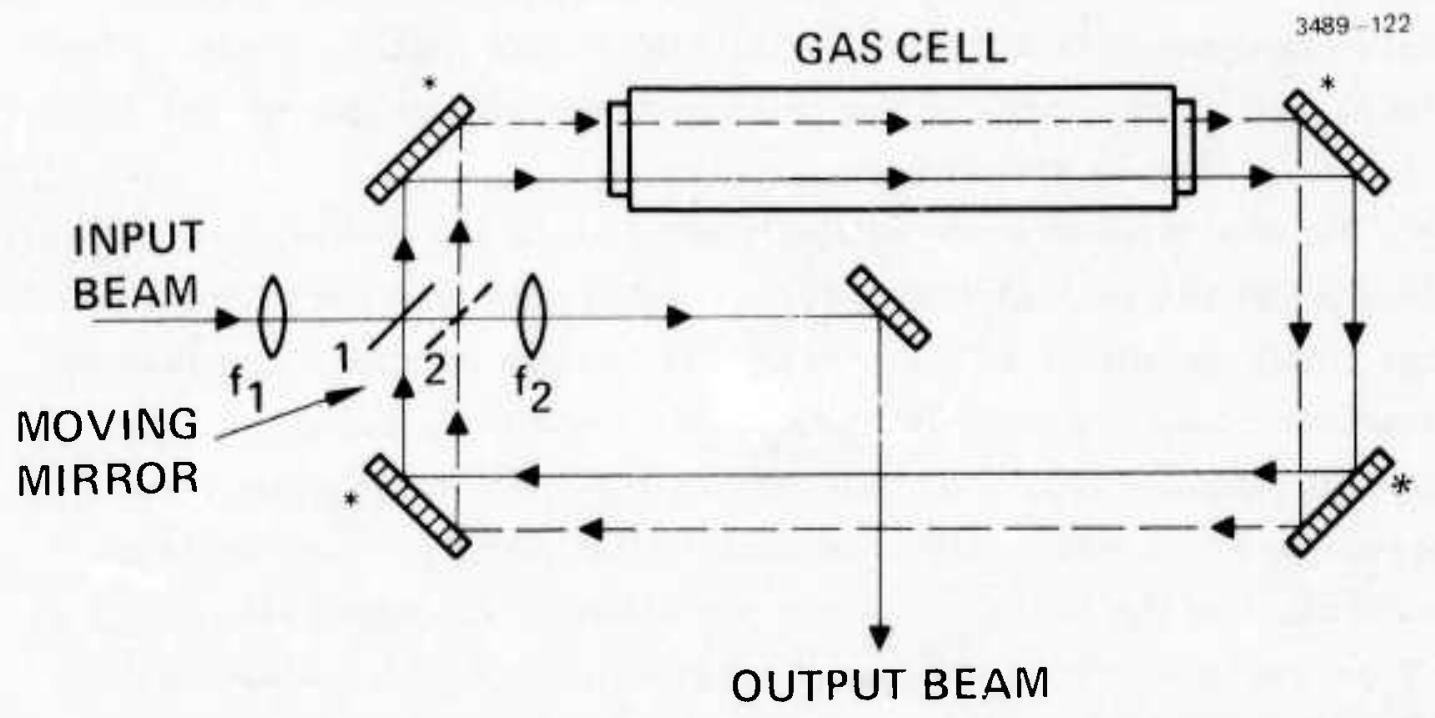

*FIXED MIRRORS

(b)

Fig. 8. Statis gas absorption cell. (a) Photograph of cell. (b) Schematic of "wind" generation apparatus. The moving mirror causes the input beam to move relative to the gas cell. The fixed mirrors bring the moving beam to the back of the moving mirror so that the output beam is stationary. 
(translational) energy in the $\mathrm{SF}_{6}$. The next choice for a buffer gas was Xe and it produced blooming according to our predictions. The disadvantage of $\mathrm{Xe}$ is its much higher cost compared with $\mathrm{SF}_{6}$.

Although the static gas cell in Fig. 8 operates satisfactorily, it has two serious drawbacks. First, it cannot be used with slewing beams because of limited input and output aperture sizes. More important, however, the optical arrangement places the blooming distortions in the last $55 \%$ of the focused propagation path. This is not only an unrealistic propagation scenario, but as discussed in Section III, it precludes observing any significant COAT compensation for blooming. To correct these deficiencies, we built a flowing gas cell 6,8 as shown in Fig. 9.

The flowing gas cell can produce transverse wind velocities up to $12 \mathrm{~cm} / \mathrm{sec}$ as measured by a hot-wire anemometer. The fill gases are again $\mathrm{Xe}$ and $\mathrm{NO}_{2}$. No turbulence effects are observed indicating $500 \mathrm{~d}$ laminar flow. This cell was used in the same vertical configuration as the static gas cell to minimize buoyancy effects although our observations have indicated that this is an unnecessary complication of the optics. The optical arrangement is similar to Fig. 8(b) except that the focusing lens can be placed much closer to the cell input window and the mirror labeled 1 or 2 in Fig. $8(b)$ is stationary.

We experienced some difficulties in using the flowing gas cell mounted as shown in Fig. 9(b). First, the blower was not vacuum tight so that small quantities of $\mathrm{NO}_{2}$ slowly leak out as it runs. The leak is too small to cause a safety hazard, but it is annoying because of the unpleasant smell of $\mathrm{NO}_{2}$. Second, the optics were cumbersome and nutmerous and had just enough residual distortion to prevent accurate measurement of the beam distortion produced by blooming alone with no COAT correction. Since natural convection effects are not noticeable for our experimental conditions, a horizontal mounting arrangement could be used. This change will probably eliminate the optics problem, but will not help the $\mathrm{NO}_{2}$ leak problem. Time on this contract did not permit us to try out a horizontal mounting, but we expect to use such an arrangement in future planned experiments. 


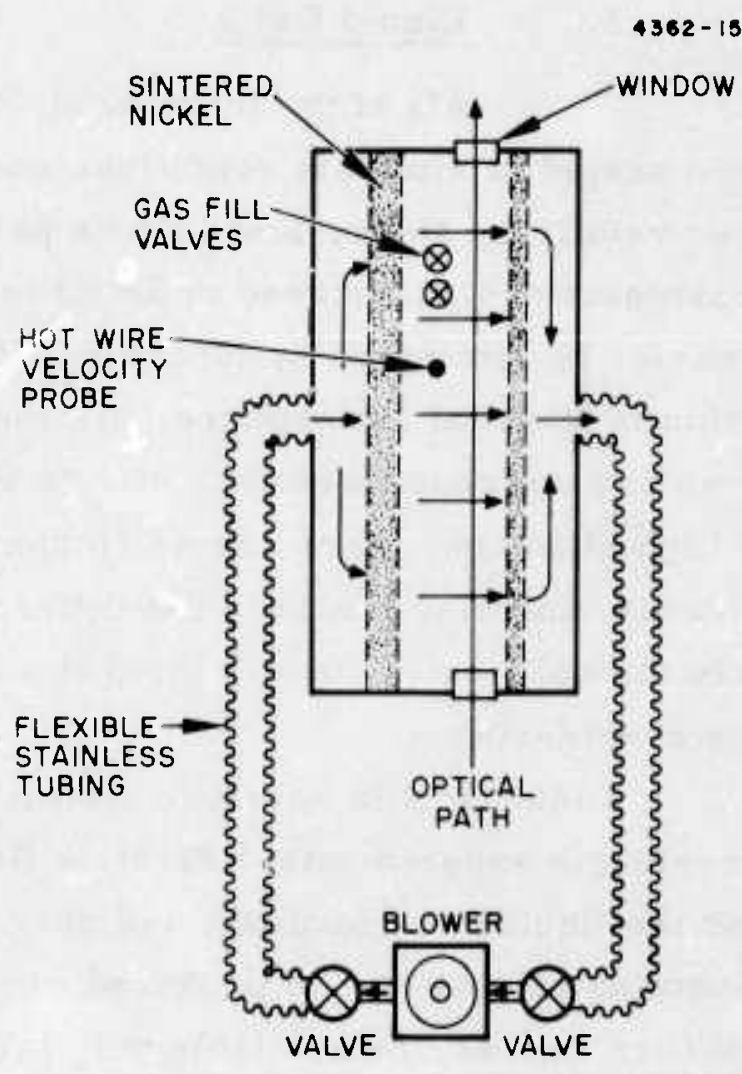

M11189

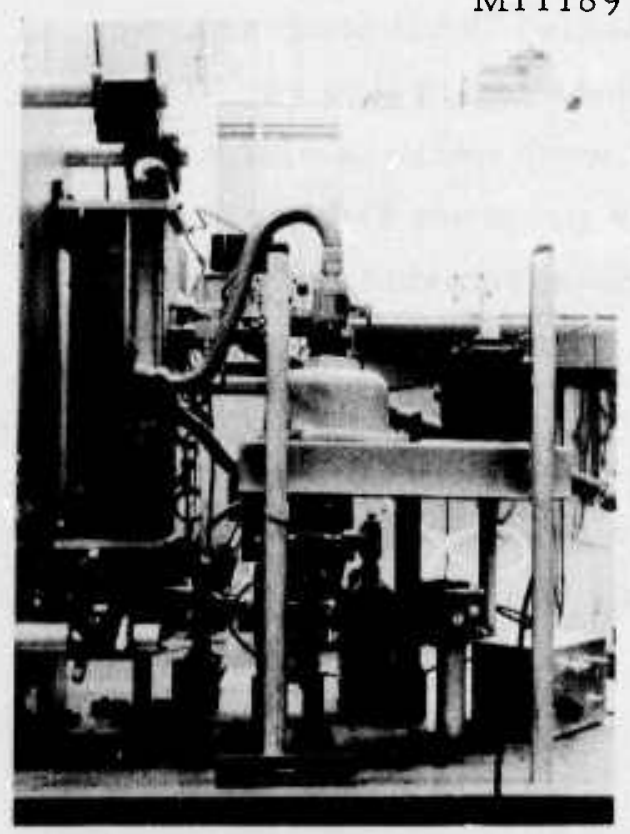

(b) Cell is mounted with a vertical path to minimize buoyancy effects.

Fig. 9. Flowing gas cell for blooming studies. 
2. Liquid Cells

All of the thermal blooming studies on this program have been scaled to simulate conditions encountered by focused beams propagating over relatively short, low altitude paths in the atmosphere. The scaling considerations, discussed in detail in Section III-A, assume that the heat transfer is dominated by forced convection (wind), which is the normal condition in the real atmosphere. As long as this assumption is satisfied, and if we are not concerned with effects like convective cooling ${ }^{9}$ that occur only at high altitudes, there are no fundamental differences between liquid and gaseous blooming media. The differences that have been observed 10 occur only for collimated-beam propagation and not for focused beams such as we are considering.

Liquid media have two distinct advantages over gases for our visiblewavelength experiments. First, a flowing-liquid cell is simple to construct and the liquids are nontoxic and easy to handle. Second, much stronger blooming effects can be produced for a given optical power and absorption because liquids are available with large values of $\mathrm{dn} / \mathrm{dT}$, the temperature coefficient of the refractive index.

As part of the Hughes Research Laboratories IR\&D program, we have studied COAT compensation for thermal blooming in liquids. The results obtained are included with this contract work because of their applicability to the questions being asked on this program and because the DARPA/RADC COAT system was used in the measurements. Two liquid cells have been used: a $1 \mathrm{~mm}$ thick static cell, and a $20 \mathrm{~cm}$ long flowing liquid cell. The flowing cell is shown in Fig. 10. We have used carbon tetrachloride $\left(\mathrm{CCl}_{4}\right)$ methanol for liquids with iodine added to absorb the $0.488 \mu \mathrm{m}$ laser light. Methanol is more convenient to use than $\mathrm{CCl}_{4}$ in the flowing cell because the very high value of $\mathrm{dn} / \mathrm{dT}$ in $\mathrm{CCl}_{4}$ makes it hard to produce a uniform, turbulence-free flow.

\section{Turbulence Phase Screen}

Generating artificial turbulence in the laboratory is not difficult, but doing it in a calibrated fashion is. We chose to use a single phase screen 


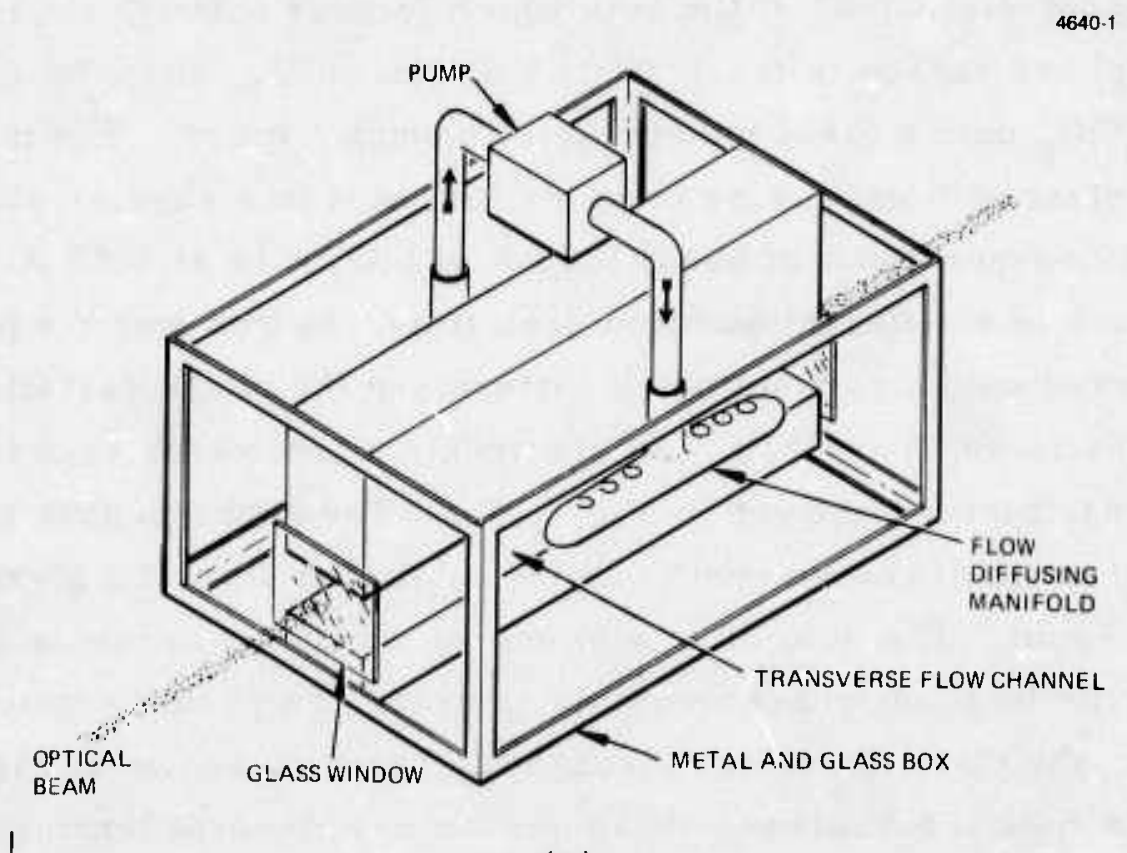

(a)

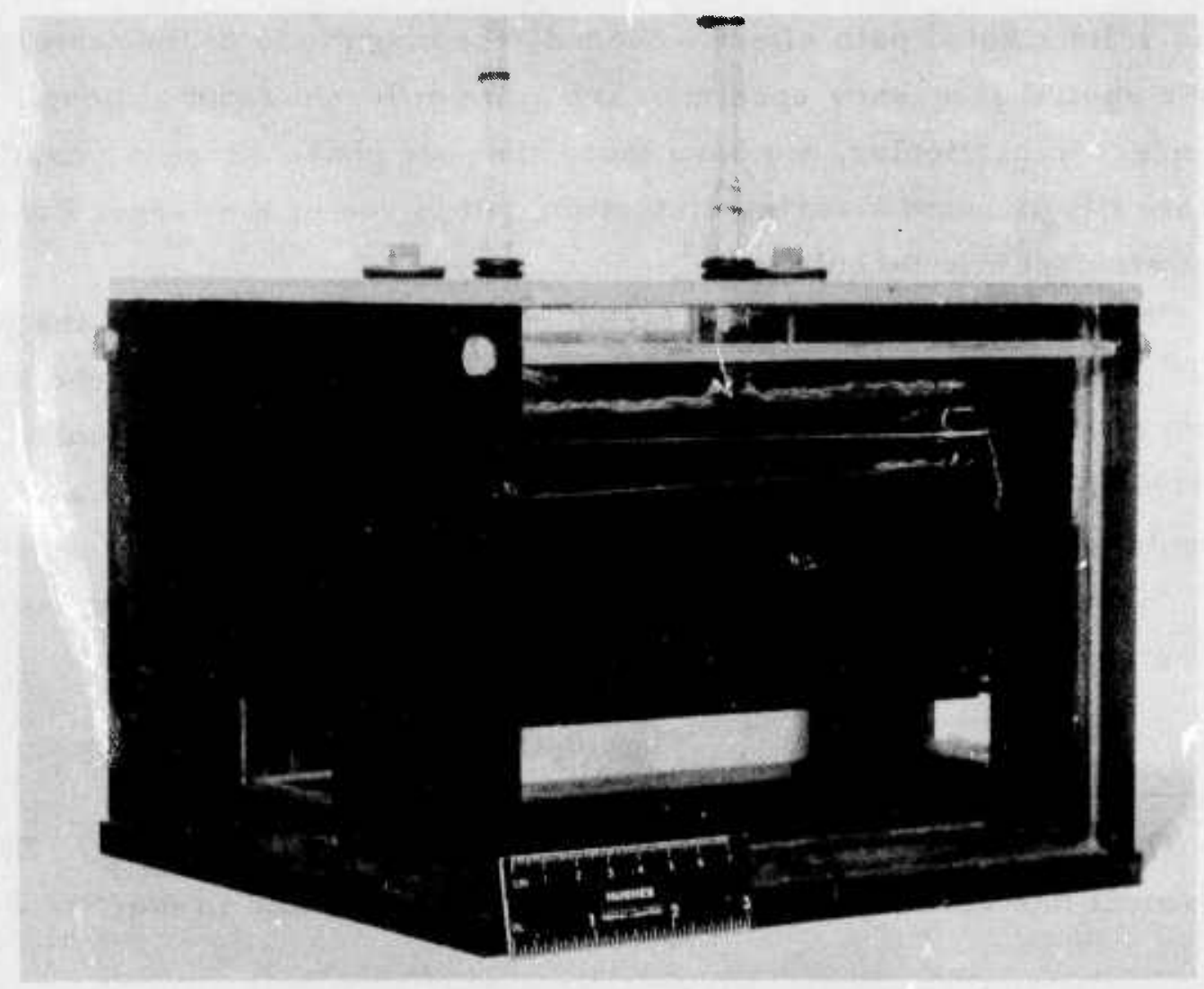

(b)

Fig. 10. Flowing liquid cell for thermal blooming studies. (a) Schematic diagram. (b) Photograph of $20 \mathrm{~cm}$ cell. 
which is placed just ahead of the lens which focuses through the blooming cell. The phase screen is fabricated as shown in Fig. 11(a) by sputtering patches of $\mathrm{SiO}_{2}$ onto a fused quartz plate through a mask. The mask is a thin metal plate with equal size holes drilled in it in a regular pattern. Three to six evaporations of about $1200 \AA$ of $\mathrm{SiO}_{2}(\lambda / 4$ at $4880 \AA)$ are made with the mask in a different position each time. In this way the plate is nearly covered with a random patch pattern and the phase variations range from zero to as much as $3 \lambda / 2$. An electronic micrometer scan through one of these phase plates is shown in Fig. 11(b). The peak-to-peak phase variation is about $3 / 4$ wavelength and the minimum size of a given "turbule" is about $0.5 \mathrm{~mm}$. The size of an element in the COAT array is also about $0.5 \mathrm{~mm}$ at the location where the plate is used. To produce time-varying distortions, the plate is rotated through the beam as shown in Fig. 11(b).

This type of turbulence phase screen has two disadvantages. First, the turbulence occurs only in one plane, unlike atmospheric turbulence which is a distributed path effect. Second, the magnitude of the distortions and their spatial frequency spectrum are quite different from atmospheric turbulence. In particular, we have found that our phase screens contain very little tilt or beam steering distortion; tili is one of the larger components of atmospheric turbulence. 11

The artificial nature of the turbulence is not important for the purposes of this program, however. We have already demonstrated the ability of COAT to correct for turbulence $e^{5,12}$ and we have shown analytically 8,13 that turbulence and blooming effects can be considered separately and then combined later to give an effective overall reduction in beam Strehl ratio.* In this contract, we are investigating the correction for turbulencelike effects in the presence of thermal blooming.

\section{E. Laboratory Optical Arrangement}

The details of the optical layout differ for each experiment. Each arrangement has several common features, however, and in functional

\footnotetext{
* Strehl ratio is the ratio of the peak beam irradiance to the diffractionlimited ir radiance.
} 

block form they all look like the schematic in Fig. 12. The first reducing telescope recollimates the transmitting array to a size usable by the BAT actuators. There are two optical paths that have identical optics and differ only in that one path has the turbulence and blooming distortions in it. The second undistorted path is required so that the COAT system can be used to remove random step phase differences introduced by the "phasor matrix." 1,2

When the photomultiplier (PMT) at location No. 2 is connected to the COAT servo system, a diffraction-limited beam is put into the path containing the DAT actuators and the distortions. The Strehl ratio with no COAT correction is measured this way. The degree of COAT correction is measured by switching the PMT at location No. 1 into the servo system. This comparison procedure is possible only if, when the blooming and turbulence distortions are removed, there is no change if the beam at the primary target plane as the PMT choice is switched from No. 2 to No. 1 and back. As mentioned previously and as discussed further in Section III, this requirement has limited our ability to measure the effectiveness of focus control alone in compensating turbulence and blooming.

For all the experiments discussed in Section III, we will use the following terminology: (1) "NO COAT correction" means PMT No. 2 in Fig. 12 is used; (2) "COAT Corrected" means PMT No. 1 is used. Occasionally, we may refer to "local loop back," meaning use of PMT No. 2 , or "target loop lock," meaning use of PMT No. 1. In all cases, the beam profile and the Strehl ratio are measured at the "primary target plane" indicated in Fig. 12 . 


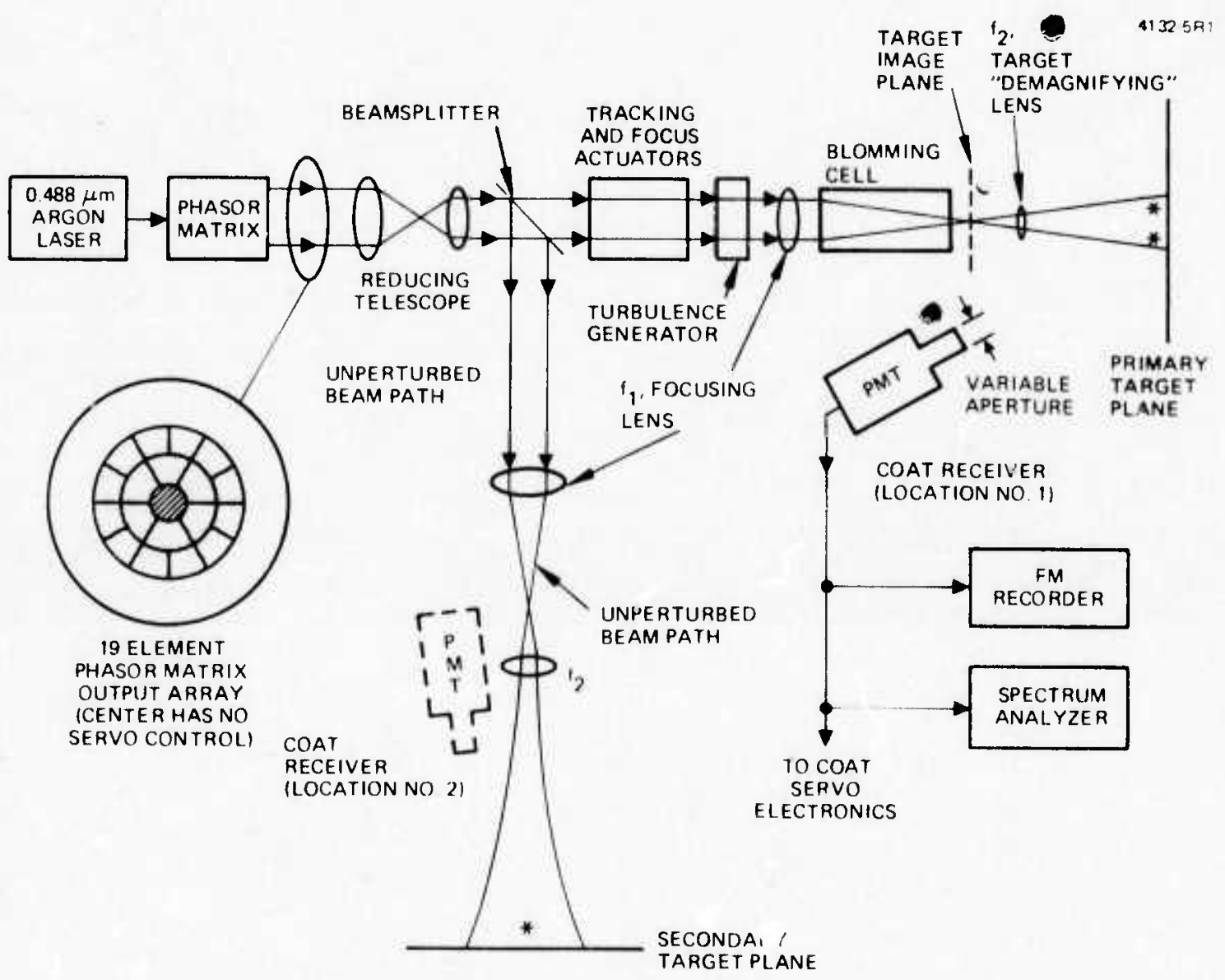

Fig. 12. Block diagram of experimental arrangement used in all COAT experiments. 
The experimental work on this program consists of five major tasks: (1) construction of a gas cell thermal blooming simulator, (2) construction of multidither tracking and focus controls, (3) measurement of COAT compensation for thermal blooming with single and multiple glint targets, (4) measurement of COAT compensation for turbulence and thermal blooming simultaneously with complex targets, (5) preliminary study of targetsignature/speckle effects on a multidither COAT system. The last three tasks also have couterparts in the analytical studies discussed in Section V. The previous section, as well as earlier reports, ${ }^{6-8}$ summarized the performance of the hardware that was built. This section summarizes the results of the experimental measurement tasks.

\section{A. Thermal Blooming Compensation}

1. Scaling Considerations

The thermal blooming experiments on this program are designed to increase our understanding of thermal blooming and of the limitations of multidither COAT systems in trying to remove these beam distortions. The experiments are thus scaled so that the results can be extended to other wavelengths, ranges, etc. Since blooming effects are most pronounced at $10.6 \mu \mathrm{m}$, we chose to scale our visible wavelength laboratory experiments to a typical $10.6 \mu \mathrm{m}$ scenario. In order to scale a $10.6 \mu \mathrm{m}$, long-path experiment to a visible wavelength laboratory experiment, three things must be considered: (1) wind speed and beam size to ensure convection-dominated heat transfer (the only case of interest with actual atmospheric conditions), (2) total absorption, and (3) blooming strength (transmitter power).

The wind speed and beam size are related to the medium properties by the Peclet number defined by

$$
P_{e}=\frac{2 V r_{o}}{\left(K / p C_{p}\right)}
$$


where $V$ is the transverse wind velocity, $r_{0}$ is the beam radius and the medium properties, $K, \rho$, and $C_{p}$ are the thermal conductivity, density, and heat capacity at constant pressure. The quantity $D=\left(K_{p} / \rho C_{p}\right)$ is also known as the "diffusivity." For almost all atmospheric conditions of interest, heat transfer is dominated by convection rather than conduction. This fact is equivalent to the statement that negligible energy is lost by a mole-cule in the time required for the molecule to move across the optical beam. Mathematically, this condition can be expressed as $\mathrm{P}_{\mathrm{e}} \gg 1$, with $\mathrm{P}_{\mathrm{e}}$ given by eq. (1). Thus for a given medium, there is a minimum value for the product $\mathrm{Vr}_{\mathrm{O}}$.

To ensure that the blooming distortiors as a function of propagation distance are the same in the laboratory at $0.488 \mu \mathrm{m}$, the total absorption, $\alpha \mathrm{L}$, and the Fresnel number, $\alpha_{F}$ must be held constant. The absorption coefficient is $\alpha$ and the total propagation distance in the blooming medium is L. The Frasnel number is conveniently defined by

$$
\alpha_{F}=\frac{\mathrm{kr}{ }_{\mathrm{O}}^{2}}{\mathrm{~L}},
$$

where $\mathrm{k}=2 \pi / \lambda$ and $\lambda$ is the wavelength.

'The blooming strength is measured ${ }^{5}$ by the produrt $\alpha_{L} \cdot P T$, where $\mathrm{P}_{\mathrm{T}}$ is the laser power at the entrance to the blooming medium and $\alpha_{L}$ is defined for a gaseous medium by

$$
\left({ }^{\alpha} L\right)_{\text {gas }}=\frac{Y_{0}(\gamma-1) k \alpha L e^{-\alpha L}}{\pi \gamma_{0} V_{p}} .
$$

The quantities $\gamma_{0}, \gamma$, and $p$ are the moleculal polarizability of the gas, the specific heat $\left(\gamma=C_{p} / C_{v}\right)$, and the static gas pressure. The absorption coefficient for the gas is $\alpha$. For a liquid, $\alpha_{L}$ is

$$
\left({ }^{\alpha}\right)_{\text {liquid }}=\frac{k \alpha L^{(\mathrm{dn} / \mathrm{dT})} \mathrm{e}^{-\alpha \mathrm{L}}}{\pi \mathrm{n}_{\mathrm{O}} \mathrm{FC}_{\mathrm{p}} \mathrm{Vr}_{\mathrm{o}}},
$$


where the liquid density is $\rho$, the nominal index of refraction is $n_{0}$, and $\mathrm{dn} / \mathrm{d} \mathrm{T}$ is the change of index with tomperature.

Wavelength and power scaling are achieved by maintaining $\alpha_{L} \cdot P_{T}$ constant. Table 3 presents a scaling example comparing $10.6 \mu \mathrm{m}$ propagation in the atmosphere to $0.488 \mu \mathrm{m}$ propagation in a $50 \mathrm{~cm}$ cell filled with $\mathrm{Xe}$ and $\mathrm{NO}_{2}$. As can be seen from Table 3, scaling to a $45 \mathrm{~J} \mathrm{kW,} 10.6 \mu \mathrm{m}$ blooming experiment can be achieved within the laboratory using reasonable experimental parameters.

As mentioned in Section II-C, we chose xenon seeded with $\mathrm{NO}_{2}$ for our experiments with gaseous blooming media. A partial pressure of only 3 to 4 Torr of $\mathrm{NO}_{2}$ is required to achieve 50 to $70 \%$ absorption at $0.488 \mu \mathrm{m}$. The cell was always operated with a total pressure of one atmosphere. We initially felt that meaningful, scalable experiments could be accomplished only with a gaseous medium, but there is no good evidence to support this

Table 3. Example of the Scaling Between a $10.6 \mu \mathrm{m}$ Atmospheric Blooming Experiment and a $0.488 \mu \mathrm{m}$ Laboratory Gas Cell Experiment

\begin{tabular}{|c|c|c|}
\hline $\begin{array}{l}\text { Experiment } \\
\text { Parameters }\end{array}$ & $\begin{array}{c}10.6 \text { ph htmospheric } \\
\text { Esperiment }\end{array}$ & $\begin{array}{l}0.488 \mathrm{~km} \\
\mathrm{SF}+\mathrm{NO}_{2} \text { Experiment }\end{array}$ \\
\hline Gas Pressure & $1 \mathrm{~atm}$ & 1 atm \\
\hline${ }^{c}{ }_{L}$ & 0.5 & 0.5 \\
\hline $\mathrm{L}$ & $2 \mathrm{~km}$ & $50 \mathrm{~cm}$ \\
\hline$r_{0}$ & $0.35 \mathrm{~m}$ & $0.13 \mathrm{~cm}$ \\
\hline V & $10 \mathrm{~m} / \mathrm{s}$ & $6 \mathrm{~cm} / \mathrm{s}$ \\
\hline $\mathrm{P}_{\mathrm{e}}($ eq. 23) & $4 \times 10^{5}$ & 20 \\
\hline${ }^{x} \mathrm{~L}$ & $2.66 \times 10^{-5}$ & $1.14 \times 10^{2}$ \\
\hline $\mathrm{P}_{\mathrm{T}}$ & $4.3 \times 10^{5} \mathrm{~W}$ & $0.1 \mathrm{~W}$ \\
\hline${ }^{a} \mathrm{~L}{ }^{P_{T}}$ & 11.4 & 11.4 \\
\hline
\end{tabular}


restriction for focused beam propagation. As data in the next section show, similar COAT compensation results are obtained with liquid and gaseous media when the experimental conditions are properly scaled. Siuce strong blooming is much easier to achieve in liquids, there is little motivation for using a gas blooming cell for future experiments.

\section{Experimental Results}

Several experimental measurements of COAT compensation for blooming have been made. The principal differences between each pair of experiments are the ratio of the length of the blooming medium to the focused propagation path and the location of the blooming medium within the path.

The first experiment was actually part of the Hughes IR\&D program. The experiment, shown schematically in Fig. 13, used a thin liquid cell filied with $\mathrm{CCl}_{4}$ doped with iodine. There was no transverse wind in this experiment. The basic two-beam arrangement described in Section II-E can be seen in Fig. 13. When the liquid cell is placed as close as possible to lens $L_{1}$, the blooming medium is in the collimated-beam, near-field region of the transmitting array. As we shall see, COAT correction has its greatest effect on near-field blooming distortions.

With the cell very close to lens $L_{1}$, the results shown in Fig. 14 are obtained. ' 4 Both the boresight irradiance and the peak irradiance in the beam are shown as a function of power into the cell. The theoretical curve shown in the figure is obtained from a simplified theory ${ }^{7,14}$ that compares the uncorrected thermally-induced beam spreading with the diffraction angle of a collimated beam. The value of the parameter $A$ is obtained by fitting the theory to the experiment at one power level. In this experiment, the transmitted beam is not focused to produce the far-field irradiance pattern, but instead expands by diffraction to produce the far-field pattern.

The data in Fig. 14 indicate that the COAT system increases the optimum transmitter power* by a factor of 2 to 3 and increases the peak target irradiance by up to a factor of 4 . More detailed data ca beam profiles have been presented elsewhere. ${ }^{7}$ We hypothesize at this point that the

*The transmitted power that gives maximum target ir radiance. 


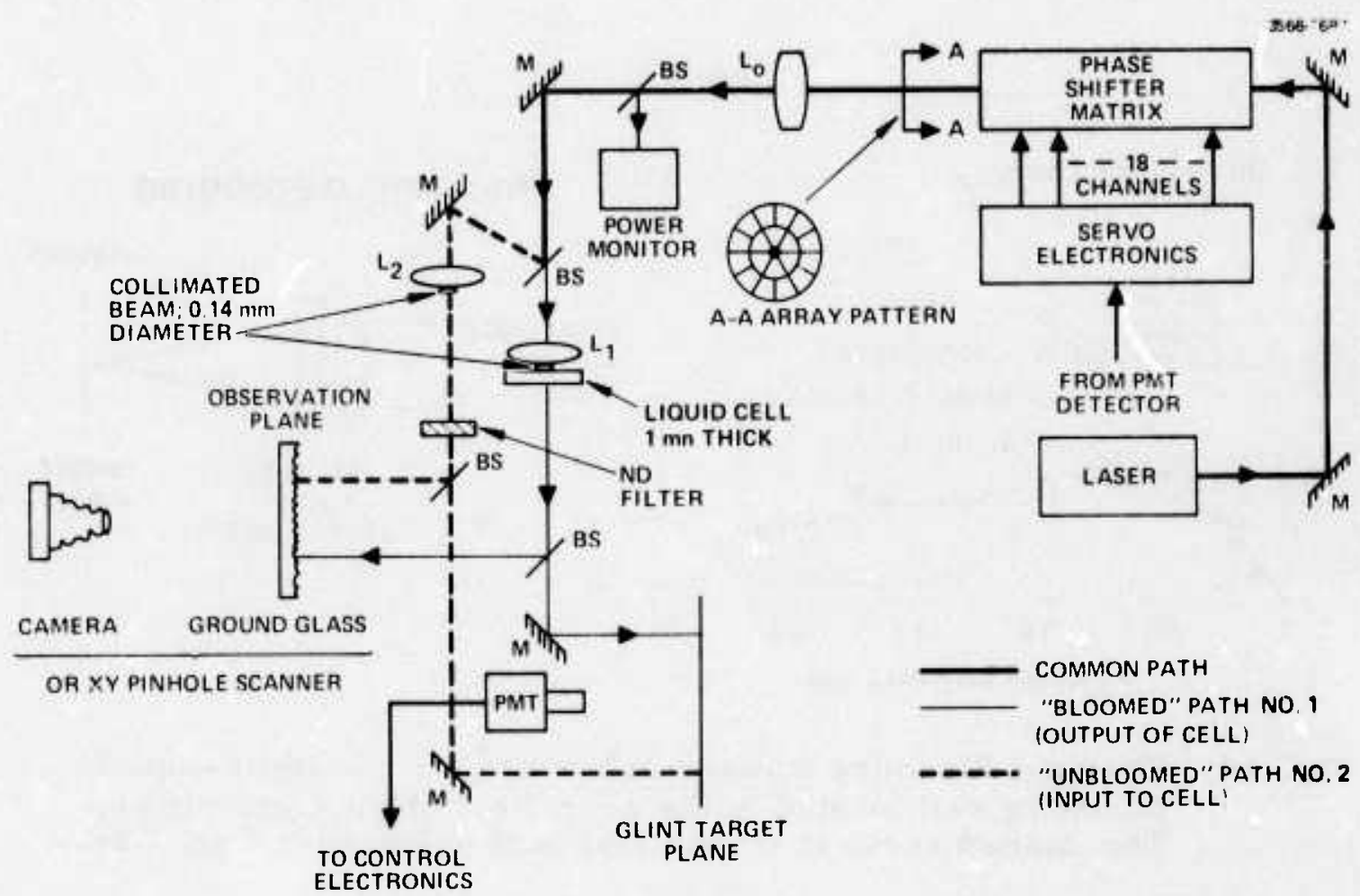

Fig. 13. Optical paths for static liquid cell blooming experiment. BS: beam splitter. M: mirror. PMT: photomultiplier. Lens $L_{0}$ together with either lens $L_{1}$ or $L_{2}$ forms a $100 x$ reducing telescope. 


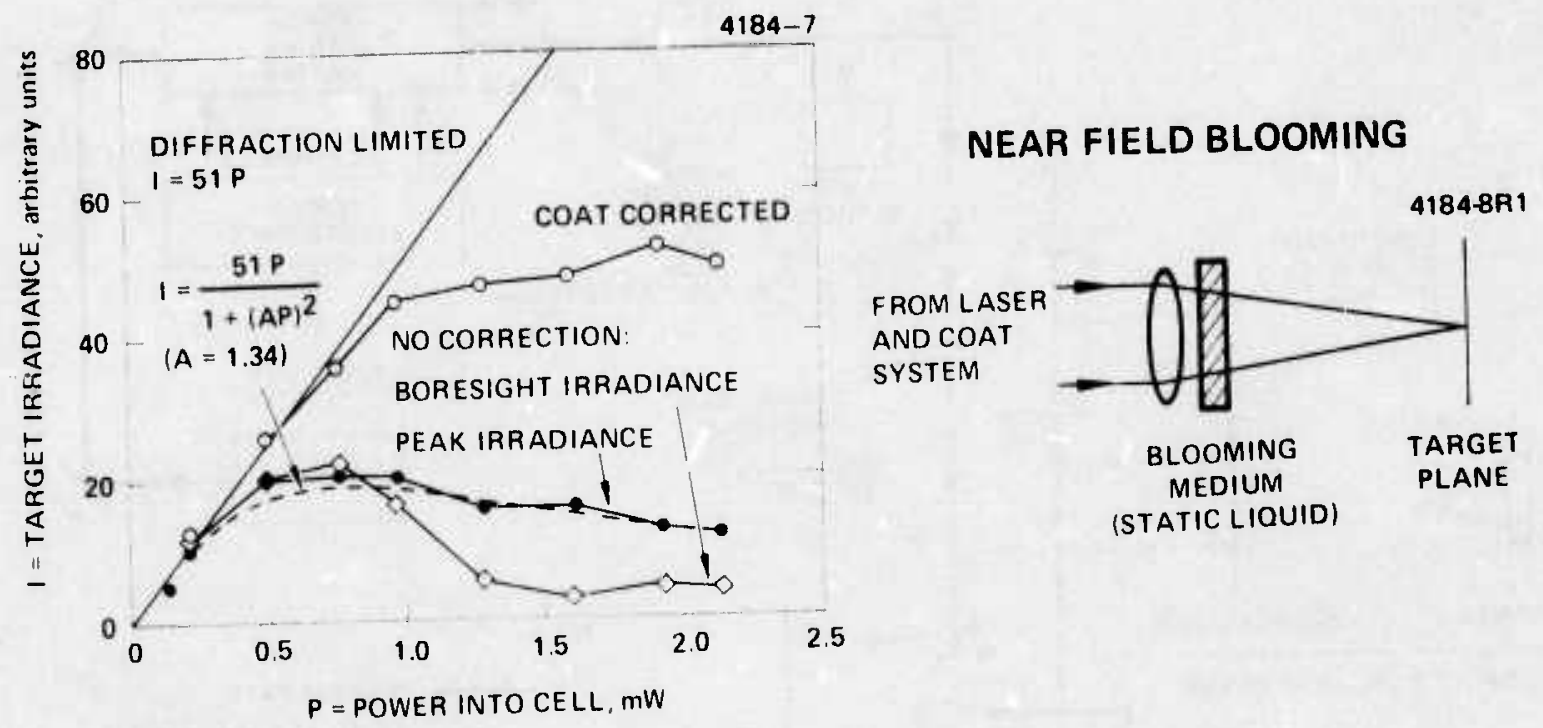

Fig. 14. Thermal blooming compensation with a thin, static-liquid blooming cell located in the near-field of the transmitter. The dashed curve is theoretical (see references 7 and 14). 
COAT system is this effective only because the blooming distortions occur in the near-field of the transmitter and that as the blooming occurs farther from the transmitting aperture, the COAT phase correction will be less effective. (In fact, a simple analysis ${ }^{7}$ can be used to show that correction in this experiment is being limitsd mainly by the finite number of corrector elements and not by a fundamental inability of COAT to remove the distortions.)

To test this hypothesis, we repeated the measurements of Fig. 14 with the cell at different distances from lens $L_{1}$. The results are shown in Fig. 15. The quantity $Z_{R}=D_{T}^{2} / 4 \lambda$ is the classical Rayleigh distance, which is the distance at which the transition from near-field to far-field occurs. For our experiment, $Z_{R}=9 \mathrm{~mm}$. As expected, the degree of COAT correction is reduced significantly as the blooming cell is moved toward the far-field of the transmitting aperture $\left(d / Z_{R} \geq 1\right)$. In fact, it appears that significant correction occurs only for distortions located well within the first $30 \%$ of the propagation distance from near-field to far-field since no appreciable correction is observed for $d / Z_{R}>0.28$.

The next experiment employed the static gas cell in the arrangement shown in Fig. 16. In this case, the laser beam is actually focused through the cell. The data obtained with this arrangement are shown in Figs. 17 and 18. The photographs in Fig. 17 are black-and-white photographs from a level-quantized color TV display ${ }^{5}$ with $1.5 \mathrm{~dB}$ per color level.

There are five principil features of these data. First, the expected crescent-shaped beam distortion is observed in Fig. 17(c) and the beam is shifted into the wind. Second, when the COAT correction is applied, the beam maximum is maintained on the boresight axis where the glint is located. Third, there is no improvement in peak irradiance when the COAT correction is applied, except for a small increase in the power through an aperture whose width equals the full-width at half-maximum of the unbloomed beam. Fourth, the system convergence time was unaffected by blooming level, being 1.5 to $2.0 \mathrm{msec}$ at all power levels. Finally, the convergence is stable at all power levels. 


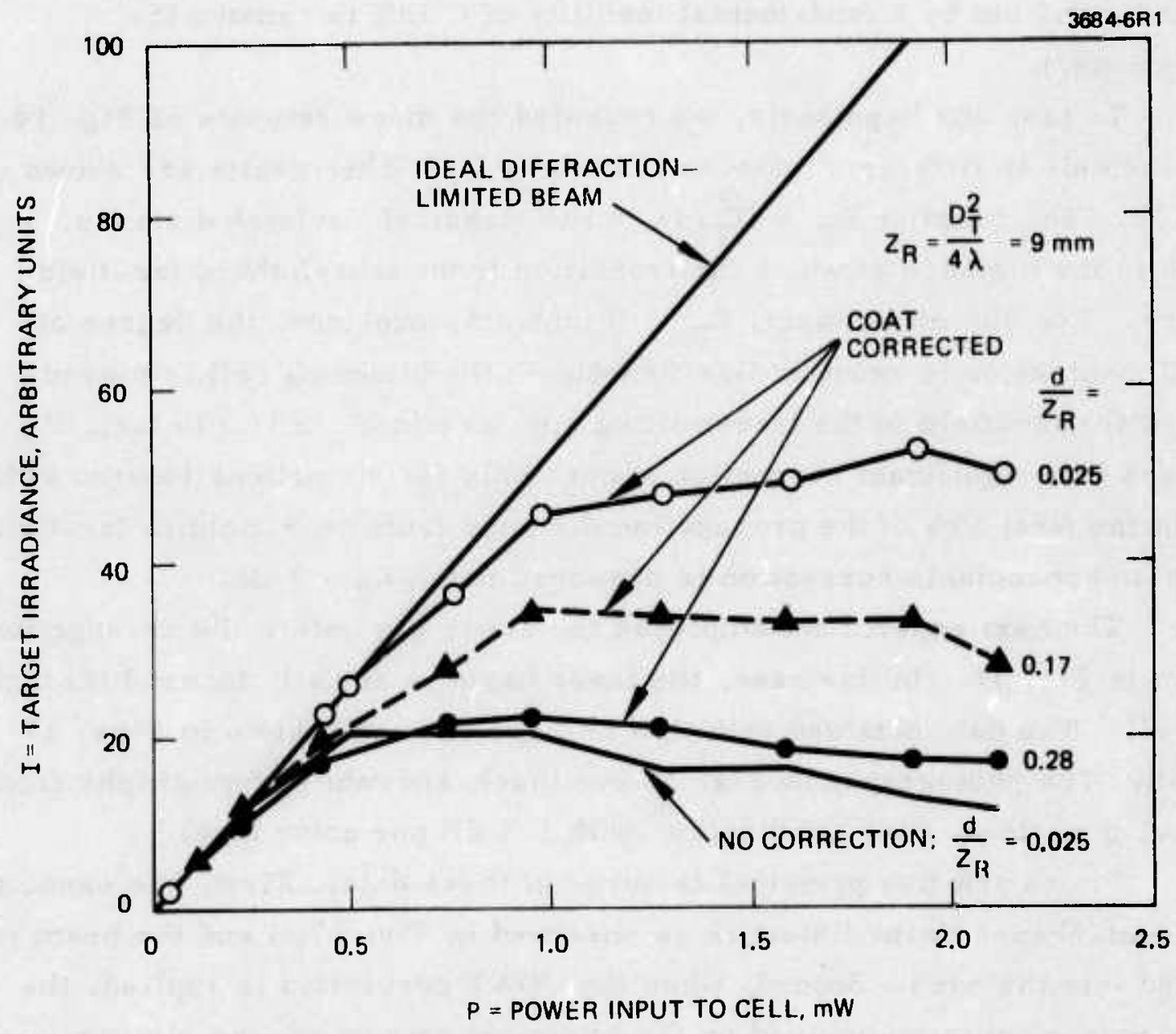

Fig. 15. Target irradiance with COAT correction for various cell locations. The quantity $d$ is the distance of the first liquid surface from the effective transmitter plane. The two curves labeled $d / Z=0.025$ are the same as the peak ir radiance curves in Fig. 14. 
36841 ค1

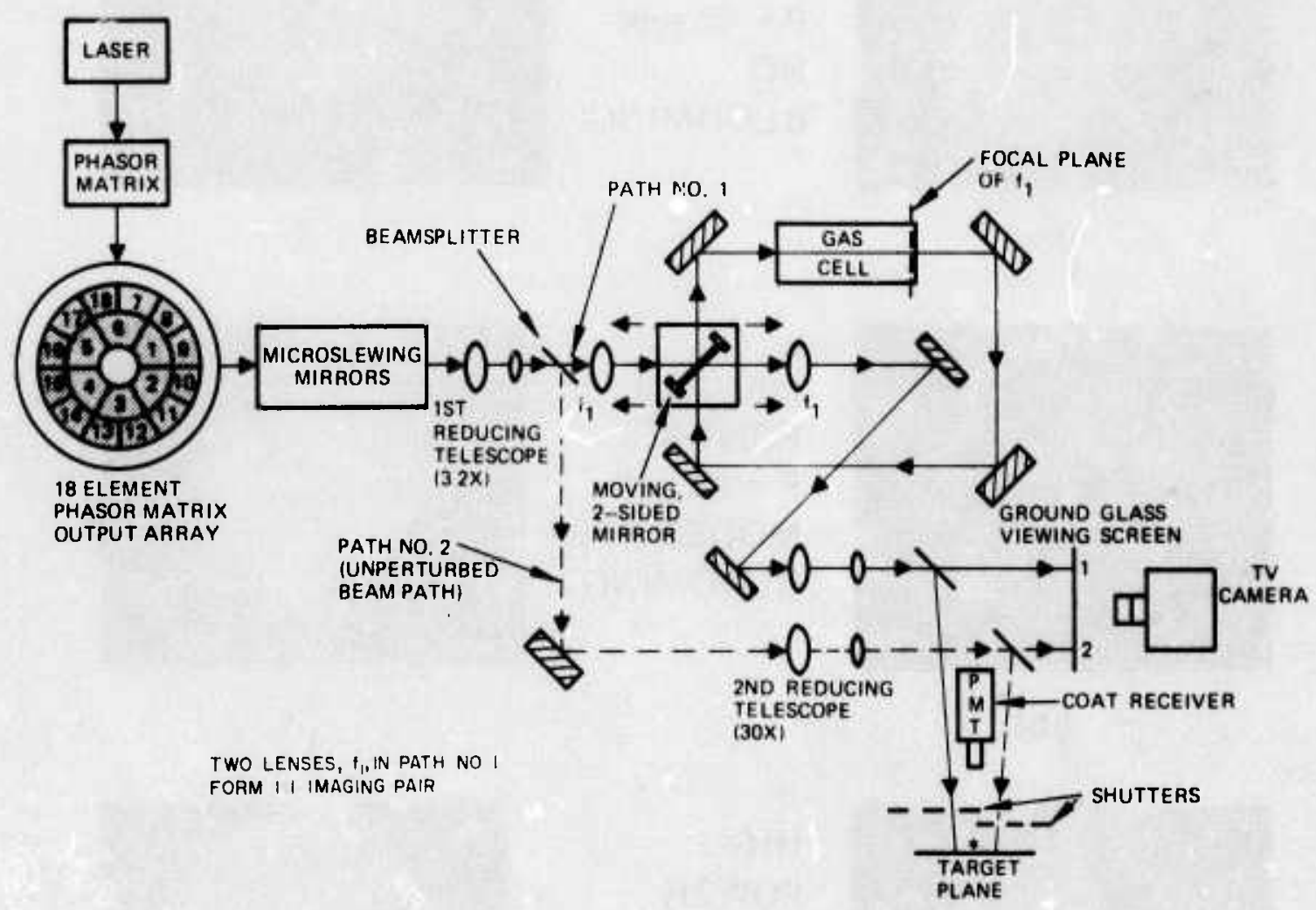

Fig. 16. Schematic of laboratory optics. Path No. 1 through the blooming cell and path No. 2 outside the cell are optically identical when the cell is not filled. 


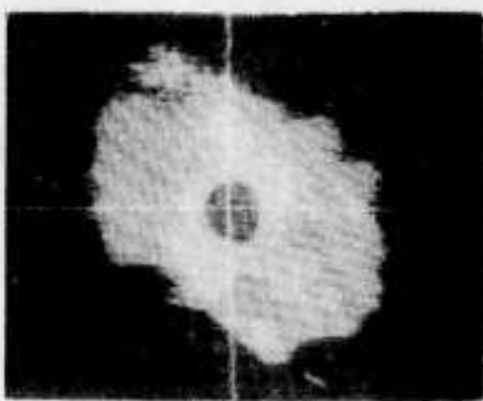

(a)

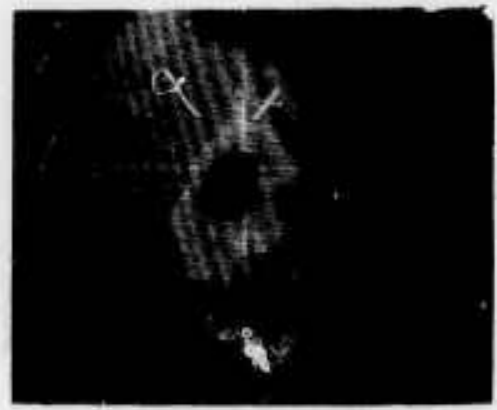

|(b)

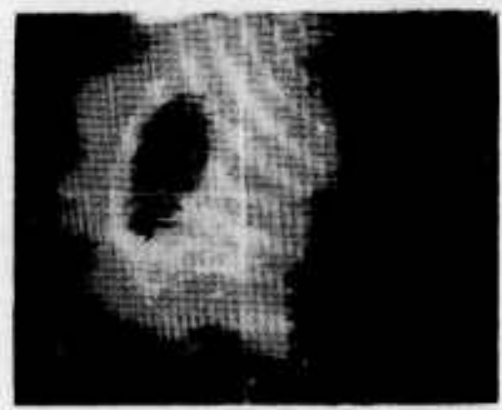

|c BORESIGHT AXIS

\section{LOW} POWER

$P=12 \mathrm{~mW}$

NO

BLOOMING

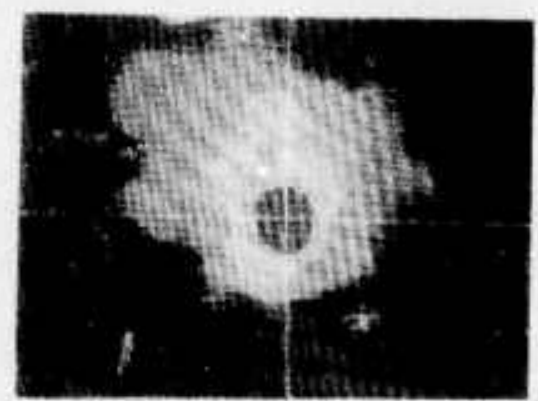

(d)

MEDIUM POWER $P=24 \mathrm{~mW}$ MODERATE BLOOMING

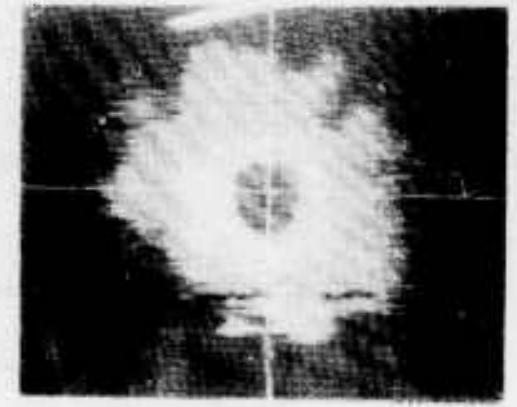

|le)

HIGH

POWER

$P=68 \mathrm{~mW}$

STRONG

BLOOMING

Fig. 17. Forced convection thermal blooming. Each shading level differs from the adjacent one by $1.5 \mathrm{~dB}$. 


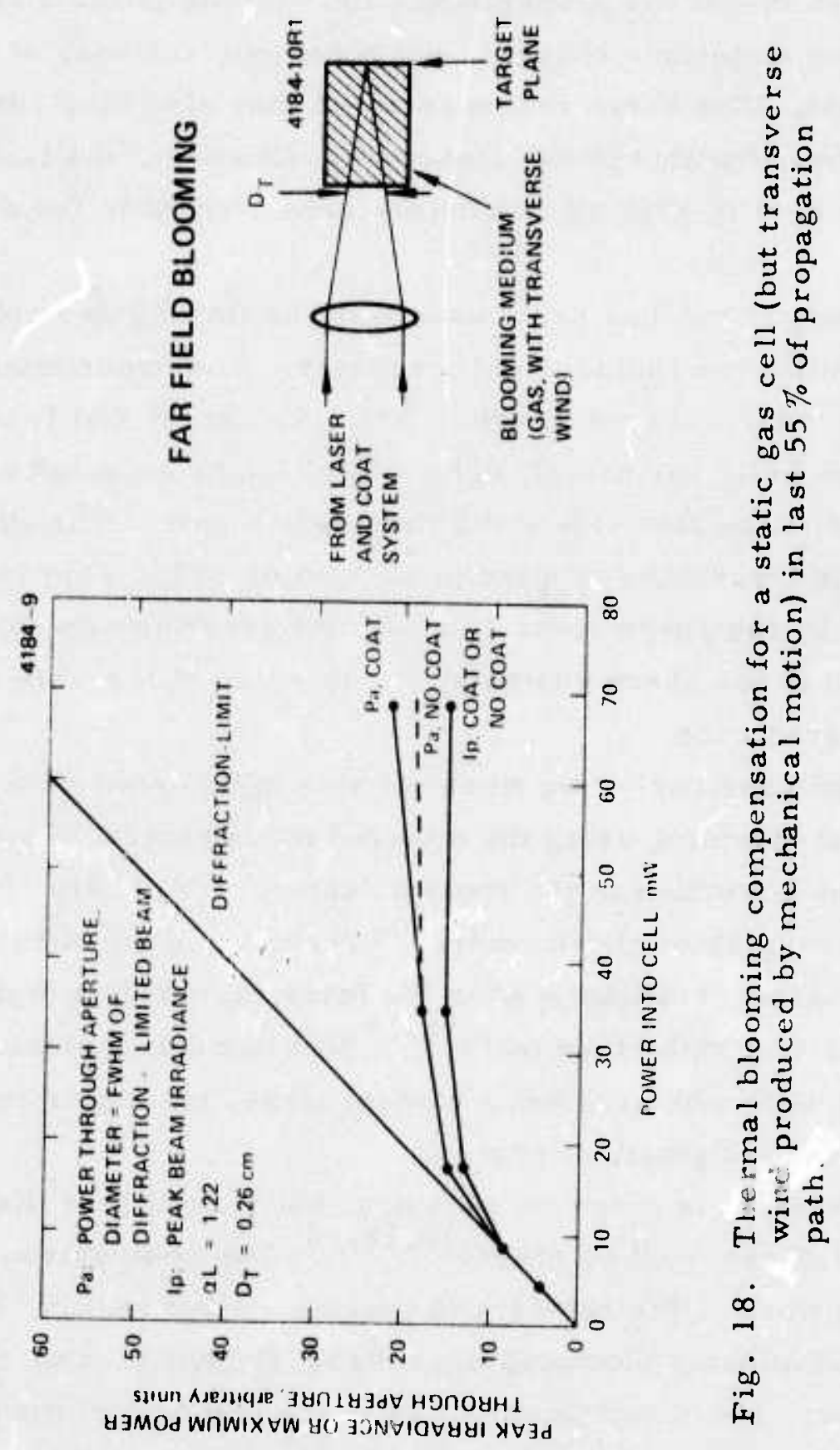


We had speculated earlier ${ }^{8,15}$ that the absence of correction in this experiment may have been caused by buoyancy effects or by too low winc' velocities. We now believe that we see no correction because the blooming medium is in the last $55 \%$ of the propagation path. Strong phase distorions in this region require amplituds changes, not phase corrections, at the transmitting aperture. The servo response speed may also play some role (see discussion below), but this is not clear now. However, the lack of COAT correction in data in Fig. 18 is entirely consistent with the data in Fig. 15.

One experimental run has been made with the flowing gas cell. The BAT tracking controls were included in these tests. The experimental arrangement was similar to those shown in Fig. 16, except that lens $f_{1}$ (focusing through the cell) was moved and its focal length selected so that the blooming occurs in the last $81 \%$ of the propagation path. The observed improvement in peak irradiance is a factor of 1.35 or $35 \%$. This improvement was produced by the phase controls alone; the tracking controls either alone or when added to the phase controls, produced no observable change in the peak target irradiance.

As mentioned previously, we were not able to determine the compensation of thermal blooming using the dithered focus control alone because of spherical distortion in the focus actuator. We did try it as an addition to the lis-channels of phase control, however, but we observed no change in the peak target irradiance when the focus control loop was closed. This result is consistent with other work ${ }^{16-18}$ that has demonstrated little or no improvement with spherical focus control alone, but significant correction with coma and astigmatism control.

This improvement is more encouraging, but is still less than the factors of 1.75 to 2.0 reported by others $16,18,20$ The comparison is unfair, however, since the work in the referenced literature used slewing beams (which effectively eliminates blooming near the focal plane). Our experiments had no slewing, and the distortion was in the last $81 \%$ of the path, thus presenting a much harder task for the COAT system. In fact, referring again to the data in Fig. 15, we observed a factor of 1.7 improvement in peak target irradiance when the blooming occurred roughly in the last $83 \%$ 
of the propagation path (compare the uncorrected curve to the corrected curve, $d / Z_{R}=0.17$, in Fig. 15). Actually, in the experiment leading to Fig. 15, the blooming occurred only from $d / Z_{R}=0.17$ to $d / Z_{R}=0.28$ rather than over the last $83 \%$ of the "focused" path $\left(\mathrm{d} / \mathrm{Z}_{\mathrm{R}}=0.17\right.$ to $\left.d / Z_{R}=1.0\right)$. Again, this is a less severe case as far as COAT correction is concerned than the experiment giving Fig. 19.

The final blooming experiment used a $20 \mathrm{~cm}$ long flowing liquid cell. Again, an optics limitation prevented us from placing the entire focused beam path within the blooming medium. The experimental arrangement is similar to that shown in Fig. 12. Two experiments were performed. In the first experiment, the blooming medium was in the last $72 \%$ of the propagation path. The results of these experiments are shown in Fig. 20. Not unexpectedly, the data are very similar to those in Fig. 19, and we observe a factor of 1.33 improvement in peak target irradiance. This result gives us confidence that there are no significant differences between focused-beam COAT compensation experiments performed with liquid and gaseous rnedia.

In the secon experiment, the liquid cell was moved to the first $72 \%$ of the path, producing the data in Fig. 21. We now see a factor of 1.5 increase in the optimum peak target irradiance. A factor of 2 increase from the uncorrected to the corrected case is seen at the highest power level. This arrangement produces blooming and compensation results along the propagation path that are similar to that occurring in a slewed beam when the entire prc, agation path is in the medium. ${ }^{16,19,20}$ Experiments using stationary and slewed beams that are focused through the entire blooming medium will be performed as part of follow-on work to this contract (contracts F30602-76-C-0021 and F30602-76-C-0022).

\section{Conclusions}

We now feel that our closed-loop, real-time phase compensation results are completely consistent with other results that were obtained using predictive, open-loop methods. $16,19,20$ A factor of about 1.5 to 2.0 improvement in peak target irradiance at optimum transmitter power is possible with multidither COAT dependong on the slewing rate. Correction factors of 2 or more may be possible at higher transmitter powers, but a lower peak target irradiance is produced. 


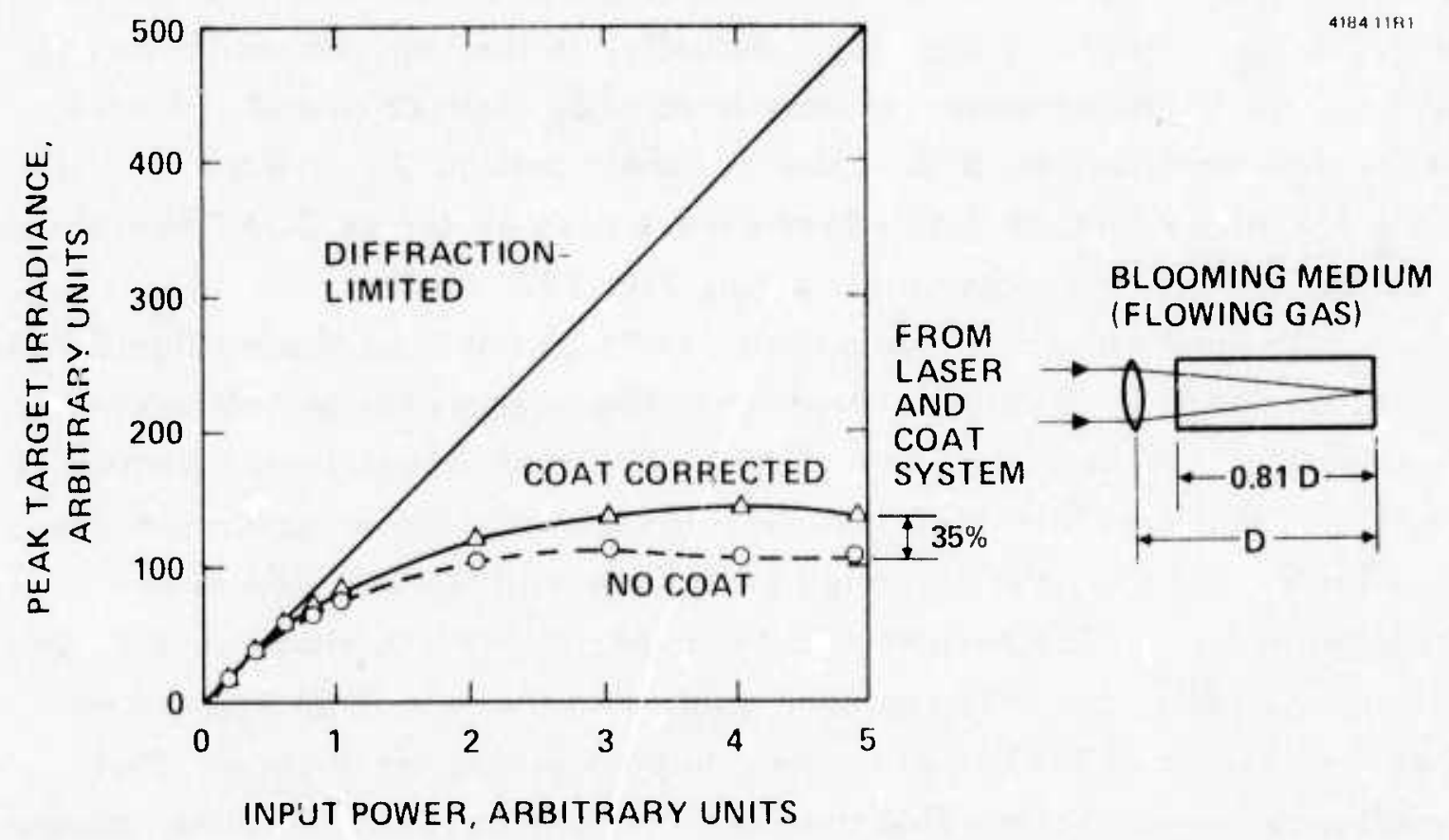

Fig. 19. Thermal blooming compensation data obtained with flowing gas cell. Also shown is the focused propagation path relative to the blooming medium.

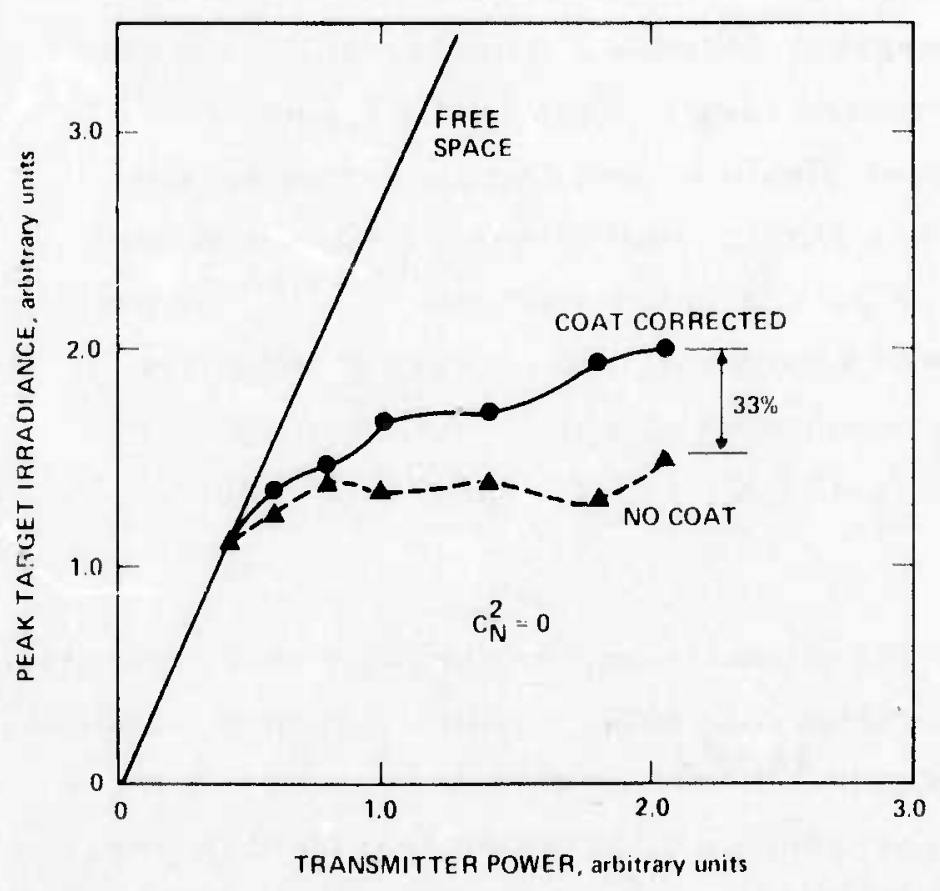

454:7R1

Fig. 20. COAT thermal blooming compensation with a flowing liquid medium. The medium is in the last $72 \%$ of the path. Results are similar to those in Fig. 19. 

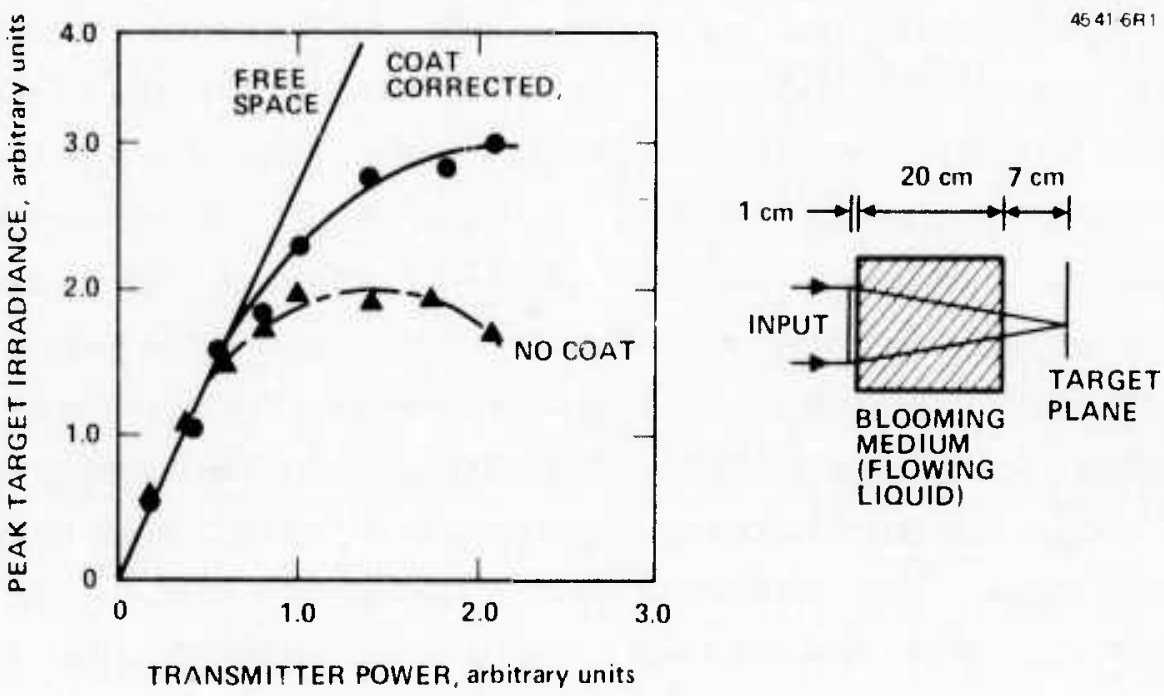

Fig. 21. COAT correction with a flowing liquid medium that is in the first $72 \%$ of the propagation path. Significantly better performance is observed than in the experiment described in Fig. 20. 
Care must be exercised when comparing compensation resultis from different experiments, since our data indicate that only the blooming distortions which occur in the first 20 to $30 \%$ of the focused propagation path can be removed by correcting the transmitter phase. This suggests that any method that can minimize the blooming distortions close to the focal plane will be effect e not only in increasing the target irradiance, but also in making the remaining distortions more amenable to COAT correction. Three teahniques come immediately to mind: (1) transmitter intensity tailoring or control, ${ }^{21-23}$ (2) use of slewed beams, ${ }^{24}$ (3) use of pulsed lasers ${ }^{25}$ with repetition rates low enough to allow only 2 to 3 pulses during a wind-driven medium transit time across the beam. When comparing improvement factors in different experiments, we must also be careful to specify whether we are comparing the maximum achievable target ir radiance with and without correction or the improvement at a fixed transmitter power. level. The use of slewing beams also greatly affects the comparisons.

In comparing our blooming experiments to COAT system tests inre with no distortion $s^{3,4}$ or with atmospheric turbulence in the path, wave found no differences in system convergence time, multiple glint discrimination, or tracking ability. We checked these quantities carefully in the last two experiments described above. We also noted that the same biooming compensation was observed with a diffuse-surface sphere for a target instead of a small point-glint target.

Although the thermal blooming distortions do not affect the $: O A T$ servo response time, we ale concerned about what the response time should be for optimum blooming compensation. Analysis of this problem is greatly complicated by the nonlinear distortion medium. At this point, let us just state the problem; we will return to it again in Section IV.

Since the COAT system-plus-blooming medium is a multiple-loop, nonlinear system, it is not unlikely that multiple solutions exist for the transmitter phase front that satisfies the servo error criterion: near-zero dither furdamental frequency in the signal reflected from the target. The prohlem is to choose the "path" in "correction space" that ensures the system stops on the true maximum target irradiance, not on a "local" maximum. It is important to point out at this juncture that we are only hypothesizing 
the existence of subsidiary maxima in the COAT convergence process with a nonlinear distortion; there is no experimertal or analytical evidence yet to support this hypothesis.

If the COAT system convergence time is faster than the response time of the nonlinear medium, it will not sample the true slope of the targetirradiance versus phase-error curve properly. That is, the instantaneous distortion will be completely removed. If instead of this fast response, the COAT system was slower than the medium, it would act like a "step, look, decide, step" servo system. In this type of system, a change in the transmitter phase front is made, the medium responds to the change, and a decision is made whether the change increased or decreased the target irradiance. If the irradiance increases, the direction of the next change is the same; if not, the direction reverses. This is effectively the type of system studied by others. ${ }^{16,19,20}$ The amplitude of the test changes (the dither amplitude in a multidither system) will aiso be important in this type of system if local maxima are to be avoided.

At this point, we speculate that it may be important to allow the medium to fully respond to the COAT-induced phase changes and possibly even to the dither frequencies if a multidither COAT system is to converge to the global optimum transmitter phase. This requirement means dither frequencies well below the present 8 to $32 \mathrm{kHz}$ baric used in the DARPA/ RADC system. The problem with such a slow system is that it is totally, inadequate for turbulence compensation. A possible solution to this dilemma, if our speculation is correct, would be the use of some sort of predictive correction so that the COAT system starts close enough to the correct phasefront that local, sub-optimal maxima are avoided. A second solution may lie in the existence of turbulence in the optical path. The random nature of turbulence, and a fast COAT system's action to correct for it, may be enough to ensure that a broad region of "correction space" is sampled so that the global optimum phase correction is always found.

It is important to point out that, all of the above discussion aside, we have never observed any servo instability in our experimental blooming compensation measurements. In each experiment, the servo gain (response time) is optimized with no blooming or turbulence distortions 
present. The distortions are then added and the laser power is increased (for blooming). In no instance did the COAT servo become unstable.

The blooming compensation may have been less than anticipated, but a stable phasefront correction was always achieved. This stah behavior is not unexpected for intensity-maximization systems such : ultidither, outgoing wave COAT, but may not be characteristic of return-wave systems such as multidither, return-wave 26 or phase-conjugate 27 or "TRIM-COAT"* systems.

\section{B. Turbulence Plus Blooming Compensation}

One of the goals of this contract, and an important consideration for any operational COAT system, is the determination of whether thermal blooming will diminish a COAT system's ability to correct for turbulence. This section presents the results of our studies of this problem.

\section{Artificial Turbulence}

The artificial turbulence phase screen was produced as discussed in Section II-D and located as shown in Fig. 12. The distortions proved to be slightly too strong for this COAT system. That is, more and smaller elements are required to fully compensate for the distortions. With no COAT correction, a time-averaged $(10 \mathrm{sec})$ Strehl ratio of 0.22 was observed. The COAT system was able to correct this beam back to a Strehl ratio of 0.60 . The phase plate was rotated to produce time-varying turbulence. A plot of the control voltage for one of the transmitting array elements is shown in Fig. 22. Significant distortions (as measured by the corrections for them) occur at frequencies ap to $30 \mathrm{~Hz}$. This is in reasonably good agreement with the $50 \mathrm{~Hz}$ spectral width observed with real atmospheric turbulence ${ }^{5}$ with this COAT system.

Using a somewhat less severe phase screen, we have compared the use of phase control to the use of tracking control only. This phase screen not only had lower phase distortions, but the quartz plate was mounted in such a way that significantly more steering error was introduced than with

"TRIM-COAT is the acronym applied to broad-band image-compensation (I-COAT) systems that are used to correct the phase of a transmitted, coherent beam. 

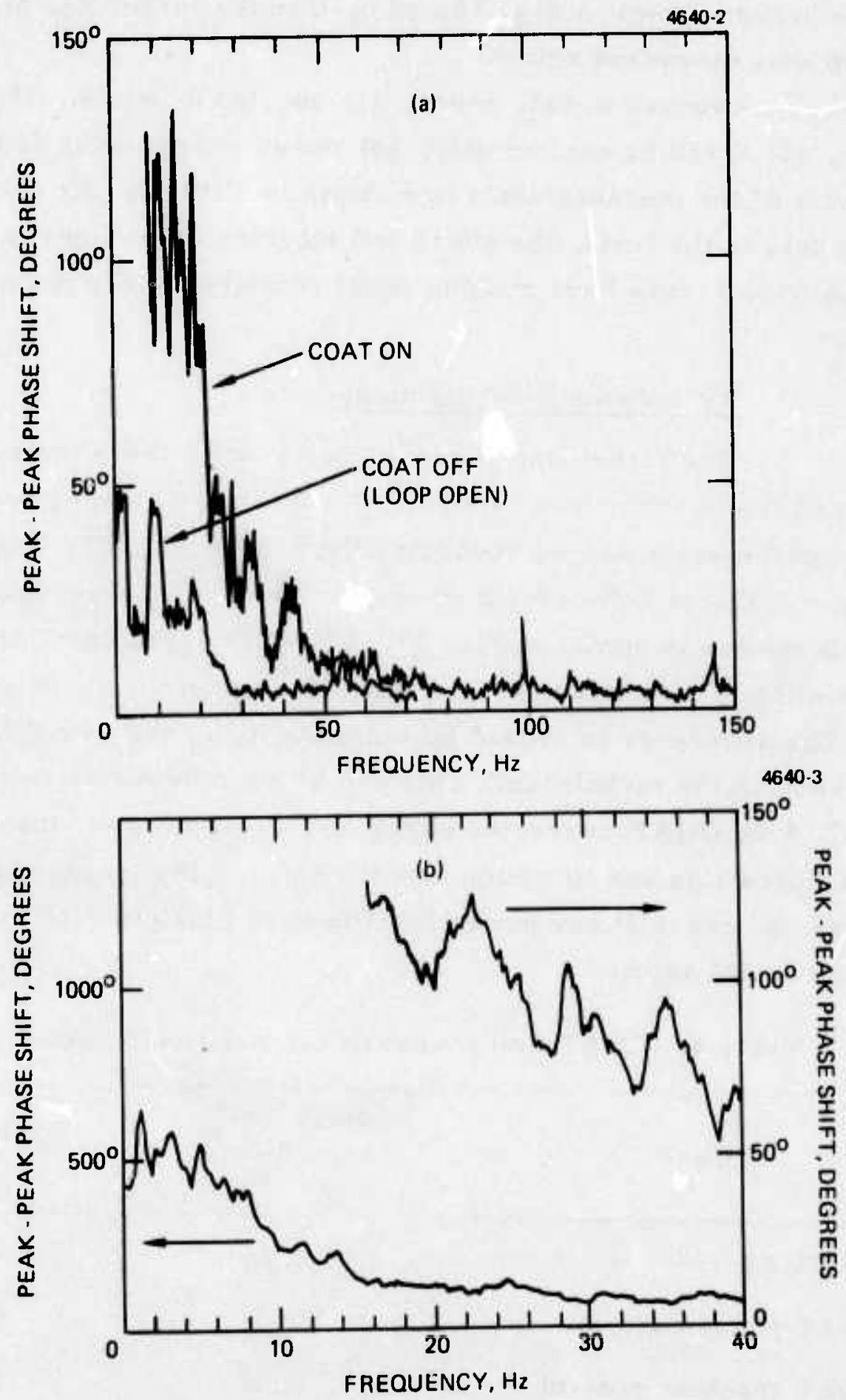

Fig. 22. Artificial turbulence frequency spectra. The observed signal is the control signal from COAT channel No. 5. (a) Total spectrum to $150 \mathrm{~Hz}$. (b) Expanded scale spectrum from dc to $40 \mathrm{~Hz}$. Note the different vertical scales for the two curve. 
the phase screen shown in Fig. 12 and used in the turbulence plus blooming measurements discussed below.

We have looked at four cases: (1) no COAT control, (2) phase control only, (3) tracking control only, (4) phase and tracking control together. The results of the measurements are shown in Table 4. As can be seen from the data in the table, the phase and steering distortions in this artificial turbulence screen have roughly equal contributions to the uncompensated distortion.

\section{Turbulence Plus Blooming}

The turbulence phase screen used in these tests was the stronger of the two discussed above. The thermal blooming scenario chosen was the same as that producing the data in Fig. 21: a flowing liquid medium in the first $72 \%$ of the propagation path. The observed compensation performance is shown in Fig. 23. The peak irradiance for turbulence plus blooming is almost as good as that achieved in the absence of turbulence. The difference is caused by the inability of the COAT system to completely remove the turbulence. This can be seen by noting that initial slope of the $C_{N}^{2} \neq 0$, COAT-corrected curve in Fig. 22 is lower than the slope of the free-space line and by noting that the ratio of the irradiances for the two corrected cases at any power level is very close to 0.60 , the no-blooming, corrected Strehl ratio.

Table 4. COAT Compensation for Artificial Turbulence

\begin{tabular}{|l|c|c|}
\hline Case & $\begin{array}{c}\text { Peak Target } \\
\text { Irradiance, } \\
\text { arb. units }\end{array}$ & $\begin{array}{c}\text { Strehl Ratio, * } \\
\text { S }\end{array}$ \\
\hline NO COAT & 0.39 & 0.65 \\
COAT phase control & 0.52 & 0.87 \\
COAT tracking control & 0.52 & 0.87 \\
Phase and tracking control & 0.57 & 0.95 \\
\hline$*$ S $=1$ is defined for no turbulence, COAT phase control. \\
\hline
\end{tabular}



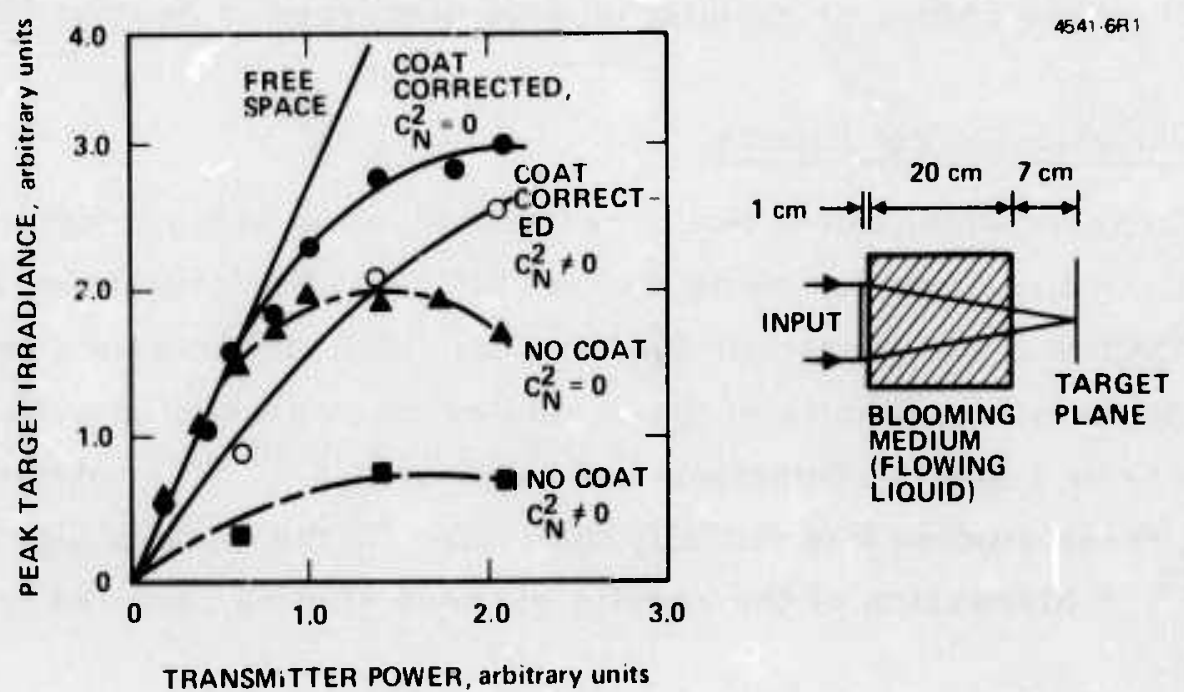

Fig. 23. COAT correction for turbulence in the presence of blooming distortions. The $\mathrm{C}_{\mathrm{N}}^{2}=0$ curves are the same as in Fig. 21. 


\section{Conclusions}

The data presented above indicate that nearly $100 \%$ of turbulence distortions can be removed by a COAT System (given a sufficient number of COAT channels) even though the absolute Strehl ratio is severely reduced by blooming. The nonlinear blooming medium does not limit the COAT system's ability to correct for turbulence. Th. conclusion is also supported by the computer simulation data discussed in Section IV.

\section{Complex Target Effects}

Contract Amendment No. 2 redirected some of the contract work to provide time and funds for some initial studies of the irteraction of a multidither COAT servo system with backscatter from an extended, semidiffuse target. Some of the results of these studies were presented at the 1975 Conference on Laser Engineering and Applications. ${ }^{28}$ The interest in pursuing these studies was initially motivated by the work of Ogrodnik and Gurski. ${ }^{23}$ A discussion of the results of these studies can also be found in Ref. 6.

\section{Problem Statement}

The physical problem known as "speckle noise" can be stated briefly. When a coherent laser beam illuminates a target, some of the energy is scattered back in the direction of the transmitted beam. With all except single glint targets, the backscattered radiation produces a random intensity pattern (a "speckle pattern") at a receiver, which is located close to the transmitter for cases of interest to us. When the appropriate conditions exist, any movement of this speckle pattern relative to the receiver will produce an amplitude modulation of the received signal. Since a multidither COAT system receiver senses amplitude modulations on the backscattered beam that are produced by dithering the transmitted beam phase, large amplitude spurious amplitude modulations in the receiver may swamp the desired modulations and thus interfere with the system operation.

Doppler shifts produced by rapidly rotating targets may also produce false signals, but these spurious signals are expected to be less important than amplitude modulation effects for the target scenarios of interest. This 
is true because the most likely glint point on a rapidly rotating target is one normal to the beam incidence direction; this point will have nearly zero transverse doppler shift. Longitudinal doppler shifts are very narrow band and thus will have minimal effect on a multiple-channel COAT system.

Thus for COAT applications, $t$ is important to determine three things about target-signature effects. First, what kind of modulations interfere with the proper operation of a multidither COAT system? The interesting parameters of the modulation are amplitude, frequency spectrum, and power spectral density. Second, what kind of receiver modulations are produced by backscatter from real targets in expected operational scenarios? That is, what scenarios will produce the modulations that can affect the COAT system? Third, can a multidither COAT system be designed that is insensitive to speckle-induced receiver modulation and if so, what are the critical design parameters? A follow-on contract, No. F30602-76-C-0021, to the current contract is designed to produce definitive answers to all these questions. Some preliminary studies of the magnitude of the effects were obtained on this contract, however.

\section{Experimental Results}

For a first experiment, we chose the simple extended moving target shown in Fig. 24(a). The target is a cylindrical scotchlite strip that could be rotated at different rates. The target is larger than the COAT formed array pattern in both dimensions (parallel with and perpendicular to the rotation axis), but exceeds the element pattern diameter only in one dimension. The curvature of the scotchlite provides a localized highlight* on which the COAT system can lock. The laboratory experimental arrangement used is shown in Fig. 12. No turbulence or blooming was introduced in the paths and the BAT controls were not used. The receiver/transmitter aperture ratio was chosen to be 0.66 .

\footnotetext{
*Jt is erroneoiss to say that a multidither COAT system requires a "glint" for convergence. It will not converge on a flat plate (at least the systems considered here will not), but any highlight a change in reflectivity with beam position on the target) will suffice to provide the required reference. We have established this fact experimentally with several target shapes.
} 


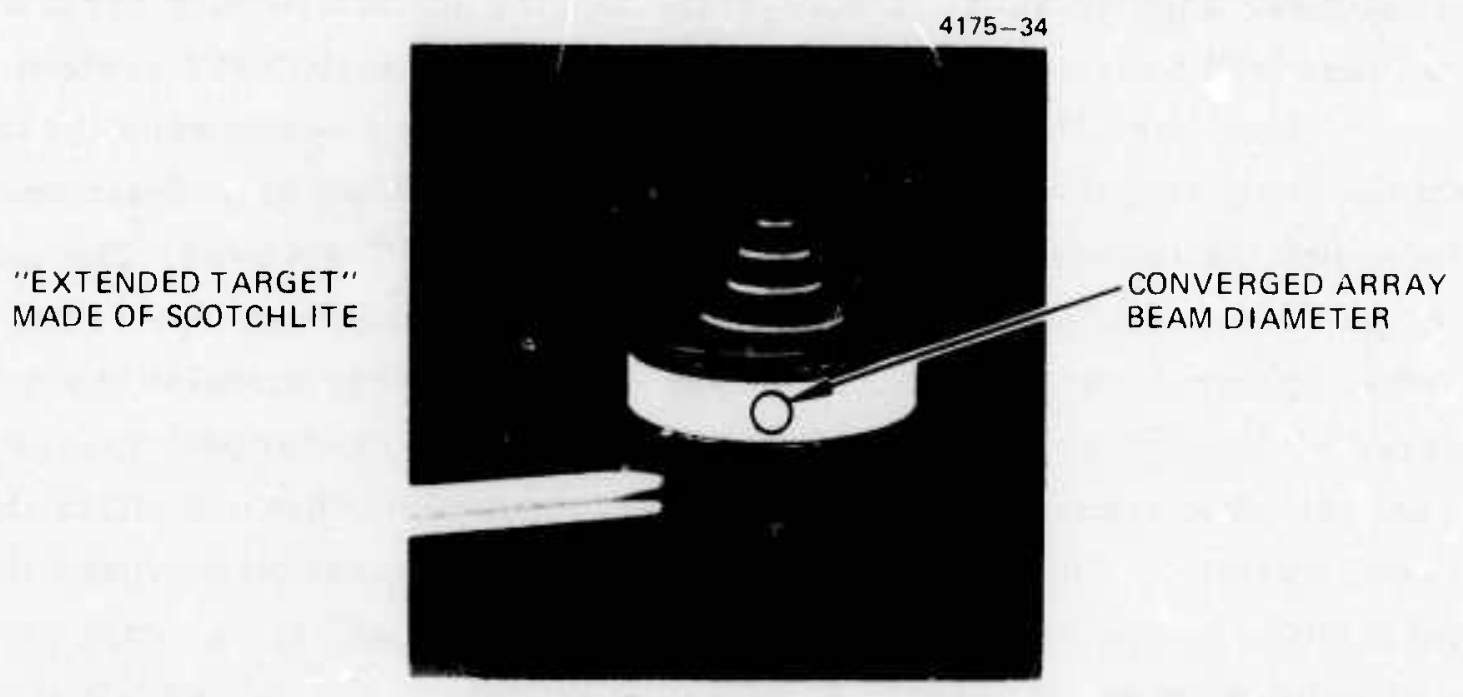

(a)

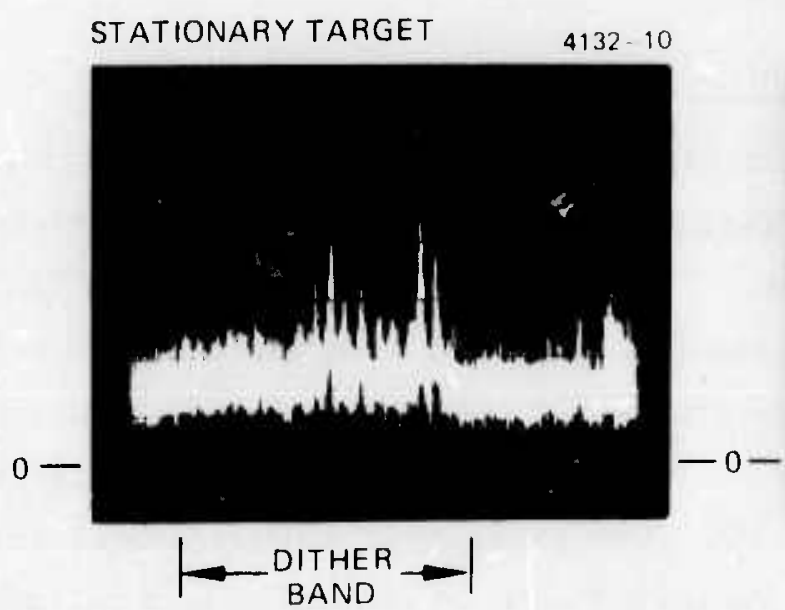

(b)

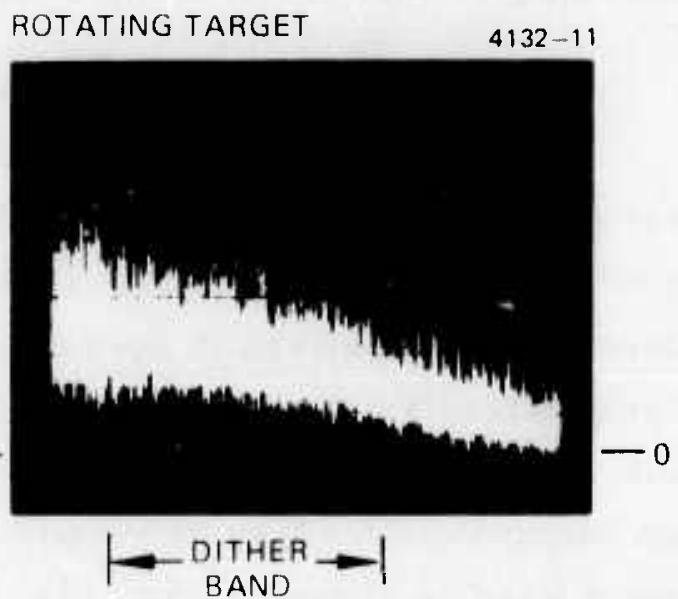

(c)

Fig. 24. COAT receiver power spectrum with extended, moving targets. (a) Extended target model. (b) Received signal spectrum with stationary target. (c) Received signal spectrum with moving (rotating) target. 
The experiment consisted of observing the peak target irradiance and the COAT receiver signal frequency spectrum when the target was stationary and moving. Figures $24(\mathrm{~b})$ and $24(\mathrm{c})$ shows the observed COAT photomultiplier receiver power spectra for a typical case. The spectrum analyzer bandwidth was $200 \mathrm{~Hz}$. Each photograph consists of five 2-min scans over a 0 to $50 \mathrm{kHz}$ range. Figure $24(\mathrm{~b})$ is the observed spectrum when the target is stationary and Fig. $24(\mathrm{c})$ is the spectrum when the target rotates. The target rotation speed was chosen to give the largest observed change in the spectrum over the dither band. For the stationary target, the noise level is low and the dither signals are clearly evident above the noise. When the target is moving, the noise level increases significantly and most of the dither signals are no longer evident. The peak target irradiance was the same, however, whether the target was stationary or moving. For this simple target, if the COAT system could converge on the target when it was stationary, the system could converge equally well when the target was moving. For every target and target motion rate that we have tried to date, this same observation has been made: no noticeable effects when the target moves.

The observation of no effect on the COAT system performance is contrary to a statement made in the third management report. The very earliest experiments did seem to show some effect. What we found, however, was that very large extraneous dither signals were being introduced into the COAT receiver by ground loops, not by target speckle effects. Since the ground loops have been eliminated, we have not been able to find a target which "confused" the COAT system solely because of its motion.

Since we have seen no deleterious COAT-system/target-modulation effects, a natural question is, What kind of spurious signals can reduce the system performance? We thus undertook an experiment whose goal was to generate large amplitude spurious amplitude-modulated signals capable of overloading the dither servo channels. In this experiment, the target was a single glint and a large receiver aperture was used. Spurious amplitude modulations were artificially introduced into the COAT system by putting an acousto-optic modulator (AOM) in the optical beam path, as shown in Fig. 25 . The AOM amplitude modulates the transmitted beam at a frequency set by 


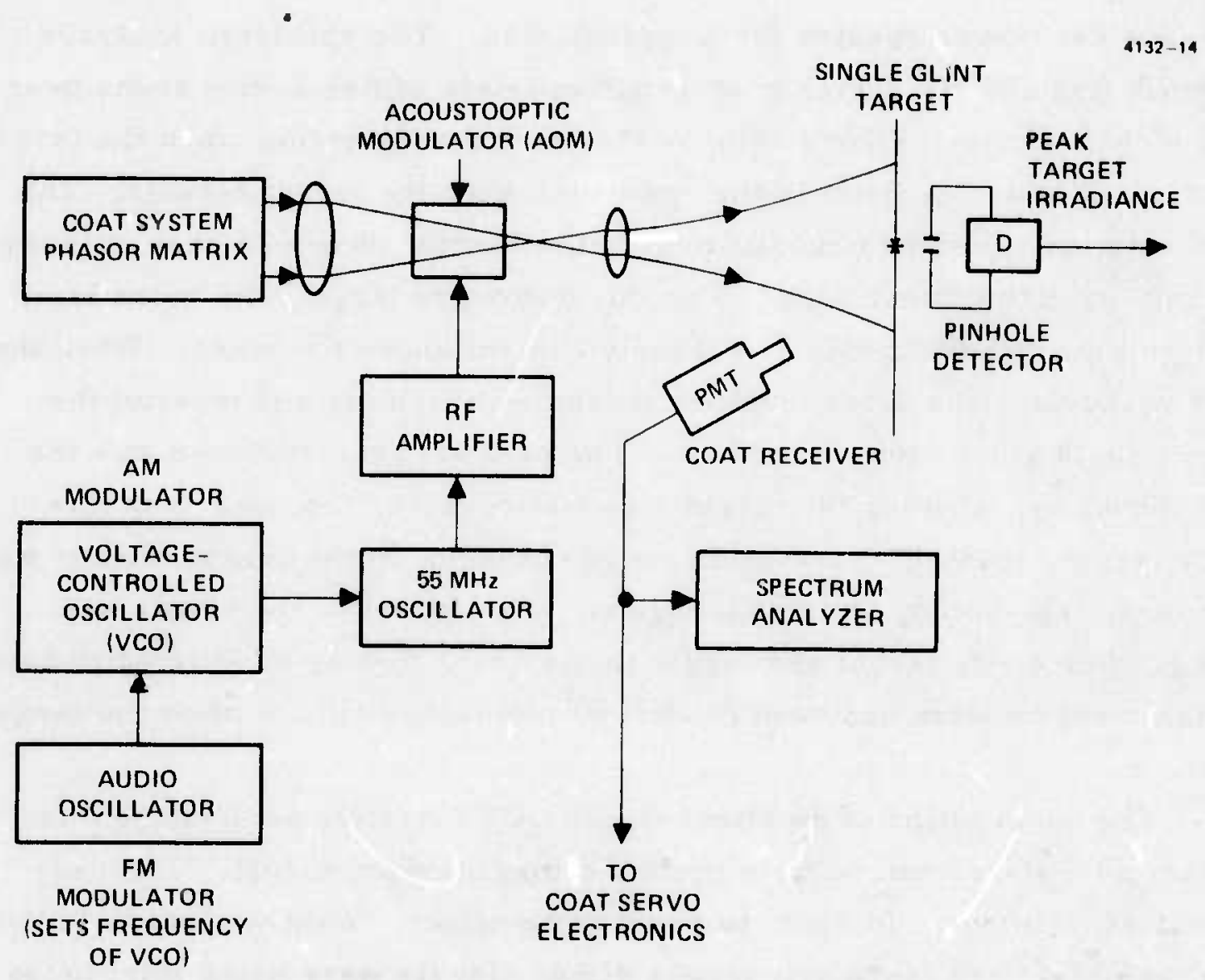

Fig. 25. Experimental setup to determine effect of spurious amplitude modulations in a COAT receiver. 
the voltage-controlled-os illator (VCO). Modulation depths of $64 \%$ were observed over the dither frequency band ( 8 to $32 \mathrm{kHz}$ ) with a triangularwave drive to the VCO.

Manually tuning the VCO through the dither passband sequentially overloaded the feedback loop at each dither frequency and caused a $10 \%_{0}$ reduction in peak target irradiance (a loss of proper phasing in 1 of the 18 COAT channels). Spectrum analyzer measurements indicated that the spurious signal level of $100 \mathrm{mV}$ generated in this mannel was much larger than the $3 \mathrm{mV}$ dither amplitudes. 'This initial result establishes the fact that external modulation applied to the COAT beam could overload the control circuitry in any single channel and cause the loss of that channel in forming the converged beam.

To roughly simulate the effect of worst-case broadband speckle modulations, the VCO was swept over a selectable bandwidth with sweep periods of $1 \mathrm{msec}$ and $0.5 \mathrm{msec}$ (shorter time than the COAT system convergence time of 1.5 to $2.0 \mathrm{msec}$ ). During this series of tests, the COAT receiver frequency spectrum was recorded and the power level in the converged beam was measured with the sweep voltage turned on and off. The center frequency, $f_{0}$, was selected for maximum effect on the COAT system. A graph of the number of active elements lost versus modulation bandwidth is shown in Fig. 26. The sweep banclwidths in Fig. 26 range from $1 \mathrm{kHz}$ to $30 \mathrm{kHz}\left(\mathrm{f}=\mathrm{f}_{\mathrm{O}} \pm \mathrm{BW} / 2\right)$, but the ratio of the peak target irradiance with the spurious modulation to that without it $\left(\mathrm{P}_{\bmod } / \mathrm{P}_{\mathrm{O}}\right)$ is nearly constant. A value of $\mathrm{P}_{\bmod } / \mathrm{P}_{\mathrm{O}}=0.69=(15 / 18)^{2}$ corresponds to a loss of proper phas ing in three channels of the average and $(14 / 18)^{2}=0.60$ corresponds to a loss of 4 channels. Similar results are obtained when the sweep period is reduced to $0.5 \mathrm{nsec}$.

Tho power spectrum of the triangulir-wave swept modulation used here consists of a sum of all the harmonics of the sweep frequency spaced about the center frequency:

$$
P_{m}=\sum_{n=-\infty}^{\infty} a_{n} \exp \left[i 2 \pi\left(f_{0}-n f_{\text {sweep }}\right)\right],
$$




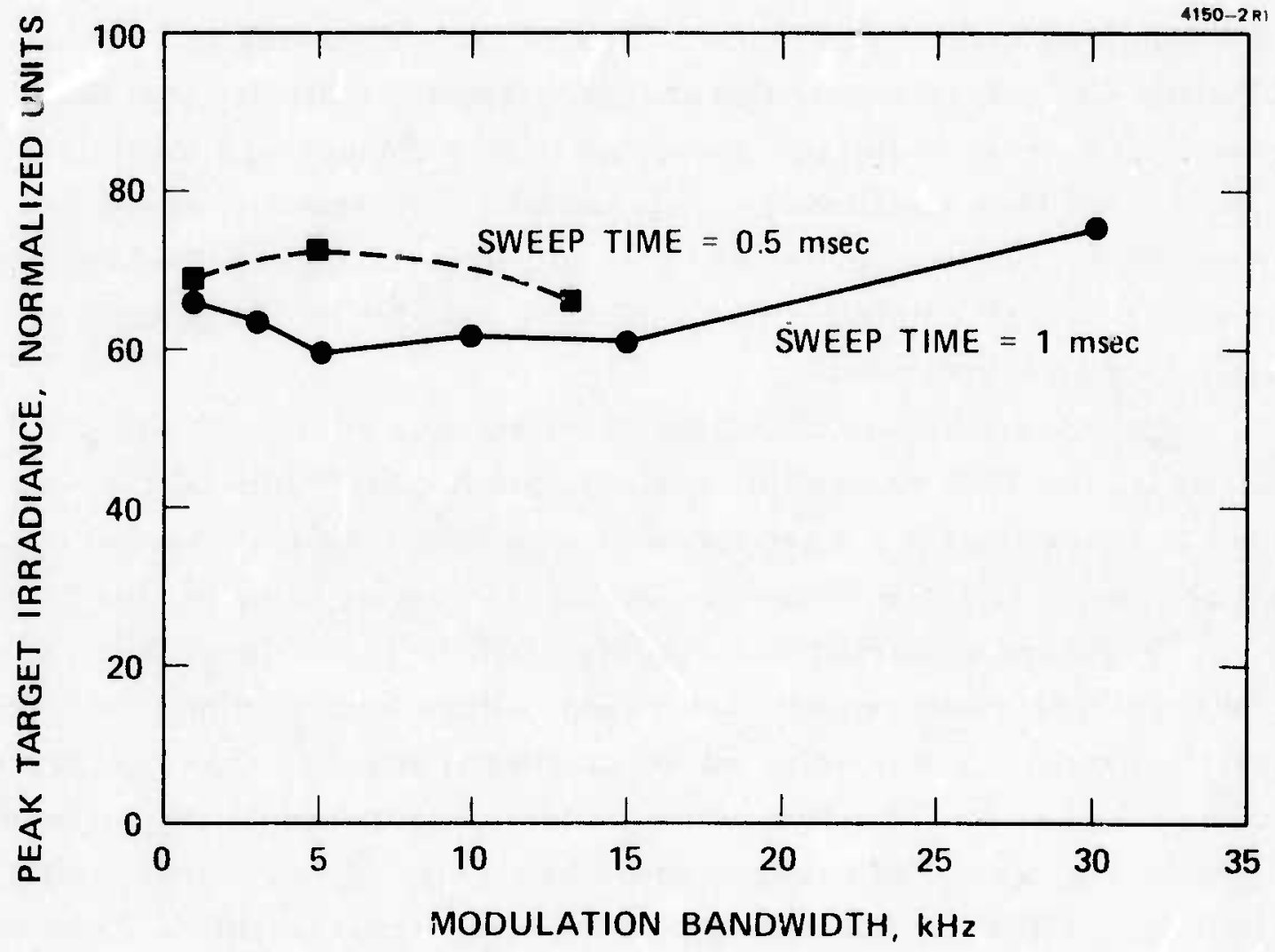

Fig. 26. Effect of a cousto-optic modulation on DARPA/RADC COAT system as a function of total modulation bandwidth. 
where the a ${ }_{n}$ are determined in part by the sweep bandwidth. Although this type of power spectrum will probably not be characteristic of any targetinduced speckle spectrum, it does have several properties which a speckleinduced receiver spectrum should al so have. First, the bandwidth of the extraneous receiver signals is limited, but will almost certainly exceed $35 \mathrm{kHz}$ in cases of most interest. ${ }^{29}$ Second, the product of peak power spectral amplitude and bandwidth is constant. What this means is that the receiver can see a $100 \%$ average modulation-depth signal at only a single frequency; as the modulation bandwidth increases, the average receiver voltage modulation denth (not the instantaneous depth or amplitude) goes down as the reciproca: of the square root of the bandwidth.

Determination of the actual spectrum of a real speckle-induced receiver signal is beyond the scope of this contract. As a worst case, however, consider a spectrum of equal amplitude sinusoidal frequencies, spaced by $100 \mathrm{~Hz}$ over a $35 \mathrm{kHz}$ bandwidth, which is close to a worst-case situation. Since there are 350 frequencies, the average receiver voltage modulation depth for any one must be $(350)^{-1 / 2} \approx 0.042$, or $4.2 \%$ to maintain a constant power-bandwidth product. This assumes a $100 \%$ speckle modulation depth, which will occur only for purely diffuse targets. If we take a $50 \%$ modulation depth since realistic targets will probably have some specular returns, the average modulation depth drops to $2.1 \%$. This value is below the $5.6 \%$ peak receiver modulation produced by a multidither system operating with a $\pm 20^{\circ}$ dither modulation.

A further consideration that we have not taken into account is that the spectral components of speckle-induced receiver noise will have randomly varying phases with respect to the dither modulation. The servo synchronous detectors will thus further discriminate against this type of noise. We are developing an analytical demonstration of the magnitude of this discrimination on Contract F30602-76-C-0021. Some of the details of the early stages of this analysis are given in Reg. 6 and in Section IV-8 of this report.

A more realistic speckle-induced spectrum might be a uniform triangular or gaussian distribution with a $50 \mathrm{kHz}$ bandwidth. 


\section{Conclusions}

These brief studies seem to indicate that target-signature effects are not going to be as severe as was thought earlier. More realistic targets and motions need to be studied, however, and other effects such as more servo channels or receiver signal-to-shot noise need to be investigated. The presence of turbulence or blooming may also have some effect. 
IV. ANALYTICAL TECHNICAL ACCOMPLISHMENTS

Much of the analytical work performed on this contract has been accomplished using computer simulation. The simulation codes model both the details of a COAT servomechanism (particularly in the case of multidithe $=$ COAT) and the details of the propagation of a coherent beam through a turbulent and absorbing medium. The codes have been developed to their present state on a variety of programs including the Hughes IR\&D program and several contracts: N60921-74-C-0249 (NSWC), F30602-73-C-0248 (DARPA/RADC), F30602-75-C-0001 (DARPA/RADC). The results of the work performed on this contract are summarized in this section.

A. Computer Simulation of COAT Compensation

1. Summary of Capabilities and Limitations of the Computer Simulation Codes

The elements of the adaptive optics computer simulation are indicated in the flow diagram in Fig. 27. The simulation contains computer routines that model the operation of an adaptive phase compensation system, the propagation of an optical beam through a turbulent and absorbing medium, and the reflectivity function of an idealized target. The adaptive system simulator consists of two main subblocks: a servo routine that can model either phase conjugate (return-wave) control or multidither (ouigoing-wave) control and a phase corrector routine that can model segmented or continuously deformable mirrors as well as tracking and focus control. The latter capability, added under Amendment No. 1 to this contract, is discussed in detail in Ref. 6. The simulation models a closed-loop adaptive system in which the control information is provided by the detection of a return-wave reflected from the target.

The effect of the atmosphere on the laser beam propagation is modeled by a time-dependent propagation code that treats the effects of both turbulence and thermal blooming. The time-dependent code is used on both the outgoing and return paths. The difference in the return calculation is that the propagation is modeled by a low-power, linear code, which includes the effects of the inhomogeneities caused by turbulence and the absorptive 


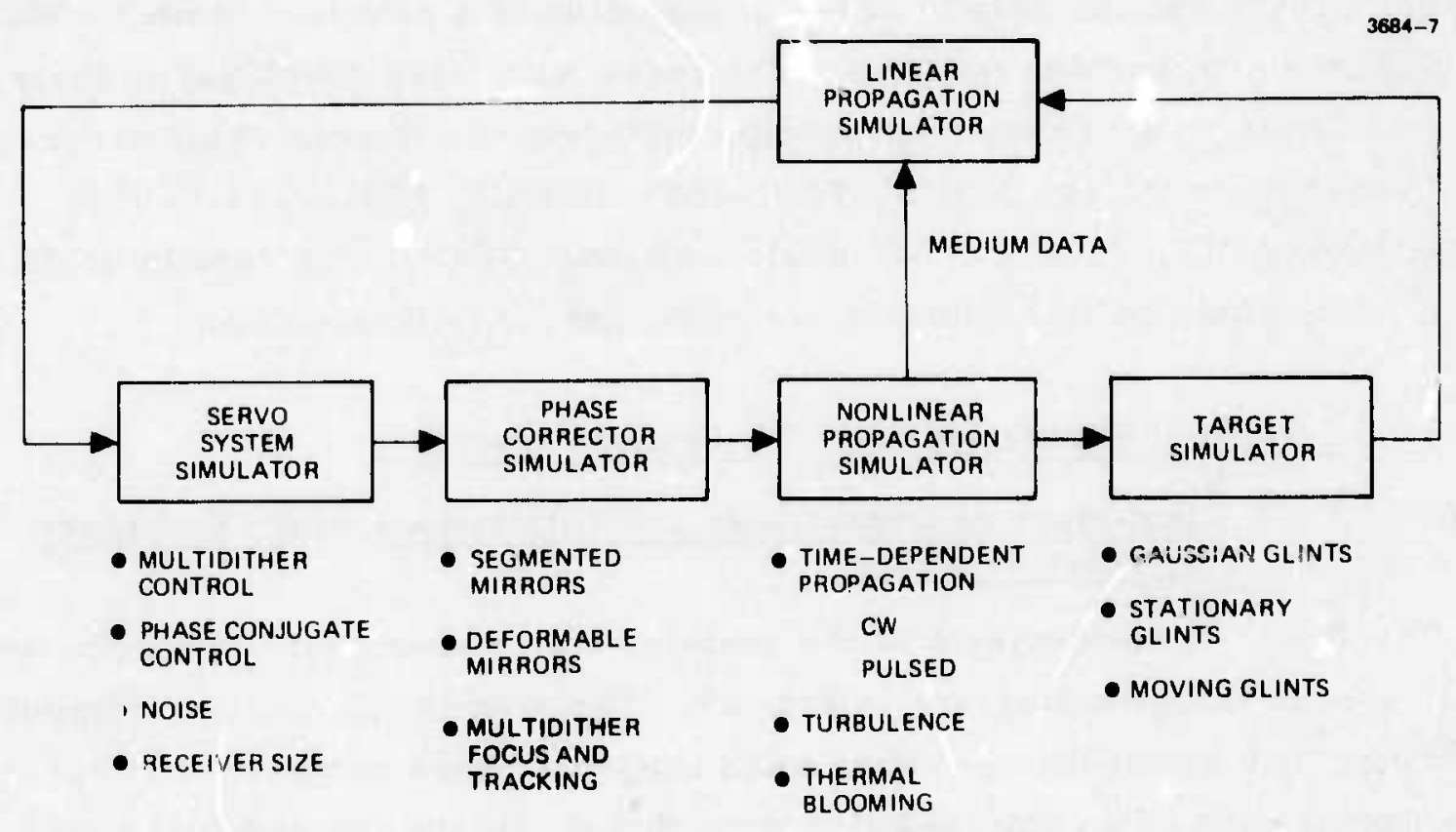

Fig. 27. Adaptive optical system simulation flow diagram. Both multidither and phase conjugate control algorithms are simulated as well as atmospheric turbulence and thermal blooming. 
heating introduced by the high-power transmitted wave; the outgoing calculation includes nonlinear heating effects (i.e., the outgoing beam affects the medium).

The time-dependent propagation algorithm used in the simulation is basically a multipulse code in which the laser energy is propagated in a series of short pulses. The pulse length is assumed short enough that no heating occurs during the pulse. Furthermore, it is assumed that the heating caused by a pulse has reached a steady state by the time the next pulse arrives. The term steady state is used here in the sense that all density gradients traveling at the sound velocity are assumed to have left the region occupied by the laser beam by the time the next pulse arrives. The only gradients present are those that are convected with the local crosswind velocity. Each pulse "sees" the medium created by the steady state heating of all previous pulses but is unaffected by its own heating. This model allows us to simulate either pulsed or $\mathrm{cw}$ propagation depending on the time between pulses relative to the time it takes an air parcel to traverse the beam. Continuous wave propagation conditions are simulated by allowing significant overlap between the regins heated by successive pulses. On this contract, we have studies only $\mathrm{cw}$ propagation.

The use of a time-dependent propagation code differs from the approach taken by Bradley and Herrmann of Lincoln Laboratory, who have used a $\mathrm{cw}$ code in their phase compensation studies. ${ }^{19}$ Although the use of a time-dependent propagation code greatly increases the complexity and cost of the calculation, we have felt compelled to use this approach for two reasons. First, the time-dependent approach is closer to the physics of the striation that we are trying to simulate since we are primarily interested in adaptive optical systems that respond in a time short compared to the time it takes the atmosphere to change. Such adaptive systems perform an essentially instantaneous determination of the required phase front. Moreover, the determination is continuously updated as the medium changes, rather than to be put in and held fixed until a steady state is reached, as is implicitly assumed when a cw propagation code is used. The distinction between the time-dependent and cw approaches is especially important for the simulation of adaptive systems that employ 
return-wave algorithms. ${ }^{30}$ whereas it can be argued that a $\mathrm{cw}$ code in conjunction with a maximization routine closely models the behavior of an adaptive optical system employing an outgoing-wave multidither algorithm, it does not appear possible to model return-wave systems with the $\mathrm{cw}$ approach since such systems do not explicitly maximize the i-radiance on the target. Instead, they continuously set the phase of the transmitted wave equal to the conjugate of the phase of the field returned from the target. There does not appear to be any reason to expect that the final state reached by such a system can be obtained from a cw calculation or a sequence of such calculations.

The other reason for the use of a time-dependent propagation code in our studies is the desire to follow the time development of the correction process especially in the case of adaptive systems employing outgoing-wave multidither control algorithms. An outgoing-wave multidither control system attempts to maximize the irradiance at the target at each instant. We wish to know if this instantaneous maximum control policy leads to a global maximum in the thermal blooming correction problem or, instead, yields a secondary maximum. This can be determined by comparing the maximum obtained with the time-dependent and the cw codes because the $\mathrm{cw}$ approach yields the global maximum.

The time sequence of events in the simulation is shown in Fig. 28. A high-power pulse of laser energy is propagated to the target and the resulting change in the density of the air in the region occupied by the beam is calculated, taking into account the convective effect of the local crosswind velocity. It is assumed that the adaptive phase compensation is applied just prior to the firing of the next high-power laser pulse. Moreover, we are presently as suming that the adaptive optical system has infinite bandwidth so that it is able to determine and apply the phase correction instantaneously without any delay or iime-averaging effects. The information required to determine the phase correction is obtained by transmitting a low-power reference pulse to the target and reflecting the same from the target. This 


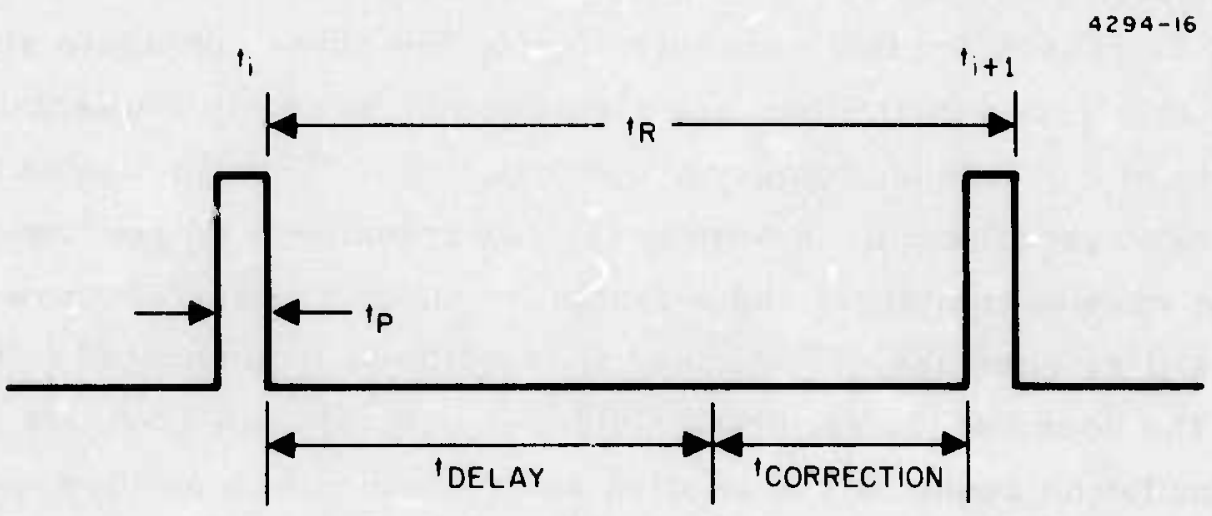

Fig. 28. Time sequence of events in multipulse adaptive optics simulation:

(1) No heating during a pulse $\left(\mathrm{t}_{\mathrm{p}}<\mathrm{t}_{\text {acoustic }}\right)$;

(2) Steady state heating for $t-t_{i} \geq t_{\text {delay' }}$;

(3) Adaptive optics correction applied in the interval $t_{\text {delay }}<t_{-} t_{i}<t_{R}$. 
reference pulse propagates through the medium that will be seen by the next high-power pulse and thus provides the necessary phase correction information. This process is then repeated for each subsequent high-power pulse.

With the exception of some runs in which the effect of the atrosphere was represented by a single phase screen in front of the tiansmitter, all of the data that have obtained to this date apply to the simulation of adaptive systems utilizing a return-wave control algorithm, i.e., systems that correct by inserting the conjugate of the phase of the wave returned from the target. The reason for this concentration on the phase conjugate simulation is simply that such simulations are significantly easier to ir.yplement and less costly to run than outgoing-wave simulations. The simulation of a return-wave system requires simply that we transmit a sirgle low-power reference wave to the target and a single low-power refle ted wave back to the transmitter aperture. The phase correction is then equated to the conjugate of the phase of the reflected field. In contrast, an accurate multidither simulation requires the sending and receiving of many low-power reference pulses for each high-power pulse. We must sample the modulations of the integrated irradiance of the target returns at a rate set by the highest dither frequency. For example, it was found in our earlier multidither servo system simulations that a sampling time increment of $5 \mu \mathrm{sec}$ is required for an 18-channel system having an upper dither frequency of $32 \mathrm{kHz}$ and an overall convergence time of $1 \mathrm{msec}$. Hence, approximately 20C samples/convergence time were required in those simulations. It was practical to lake 200 samples/convergence lime in the earlier work because of the simplicity of the propagation model that was used. In the present case, however, the propagation model is quite complicated and relatively costly to run and thus it is clearly not practical to do an equivalent simulation. Additional details on the computer simulation codes - servo, propagation, mirror, and target-are given in Ref. 30 .

\section{Turbulence and Blooming Compensation}

We have demonstrated elsewhere $e^{30}$ that the effect of atmospheric turbulence on the ensemble-averaged irradiance distribution can be accounted for by introducing a random phase screen in front of the transmitting 
apertur:. The appropriate phase distribution is that which would be produced by a point source located on the target. ${ }^{*}$ This inplies that an ideal adaptive optics system should be able to completely compensate for the effects of the turbulence by introducing the conjugate phase distribution

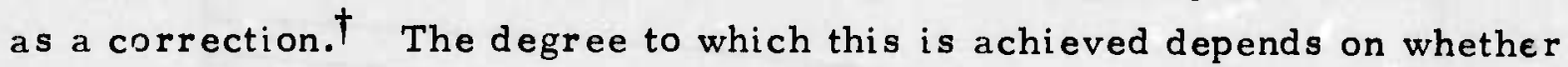
the target has a point-glint scatterer that provides the requisite phase information and on the degree to which the adaptive optics system can reproduce the desired phase distribution.

Previously ${ }^{30}$ we have demonstrated that phase conjugate COAT control with a deformable mirror can correct a beam focused through a turbulent atmosphere back to within 70 to $95 \%$ of the diffraction-limited (freespace) target irradiance. We have also demonstrated analytically ${ }^{30}$ that a multidither COAT system will arrive at the same phase correction as a phase conjugate system when correcting for linear (not power-dependent) distortions. The amount of correction depends on the range $(Z)$, turbulence strength $\left(C_{N}^{2}\right)$, wavelength $(\lambda)$, transmitter diameter $\left(D_{T}\right)$, and number of deformable corrector mirror actuators elements $\left(\mathrm{N}_{\mathrm{a}}\right)$. The dependence oi the residual phase er : or after correction is described by 6,30

$$
\left\langle\phi_{e^{2}}^{2}=0.051\left(\frac{2 \pi}{\lambda}\right)^{2} C_{N}^{2} \mathrm{ZD}_{\mathrm{T}}^{5 / 3} \mathrm{~N}_{\mathrm{a}}^{-5 / 6}\right.
$$

where $\left\langle\phi_{e}^{2}\right\rangle$ is the residual mean square phase error across the aperture after correction. This result includes an approximation for the shape of a deformable mirror surface. The effective Strehl ratio is given by

$$
S \approx \exp \left[-\left\langle\phi_{e}^{2}\right\rangle\right]
$$

The value of $\mathrm{S}$ is plotted in Fig. 29 versus $\mathrm{C}_{\mathrm{N}}^{2}$ for severa! cases of interest.

\footnotetext{
*In this discussion, we assurne that amplitude scintillation effects are small. If they are not small, the effect of atmospheric turbulence cannot be represented simply by a phase screen but rather we must also introduce a random apodization.

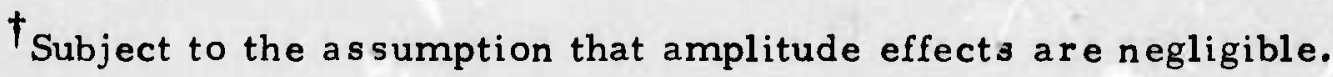




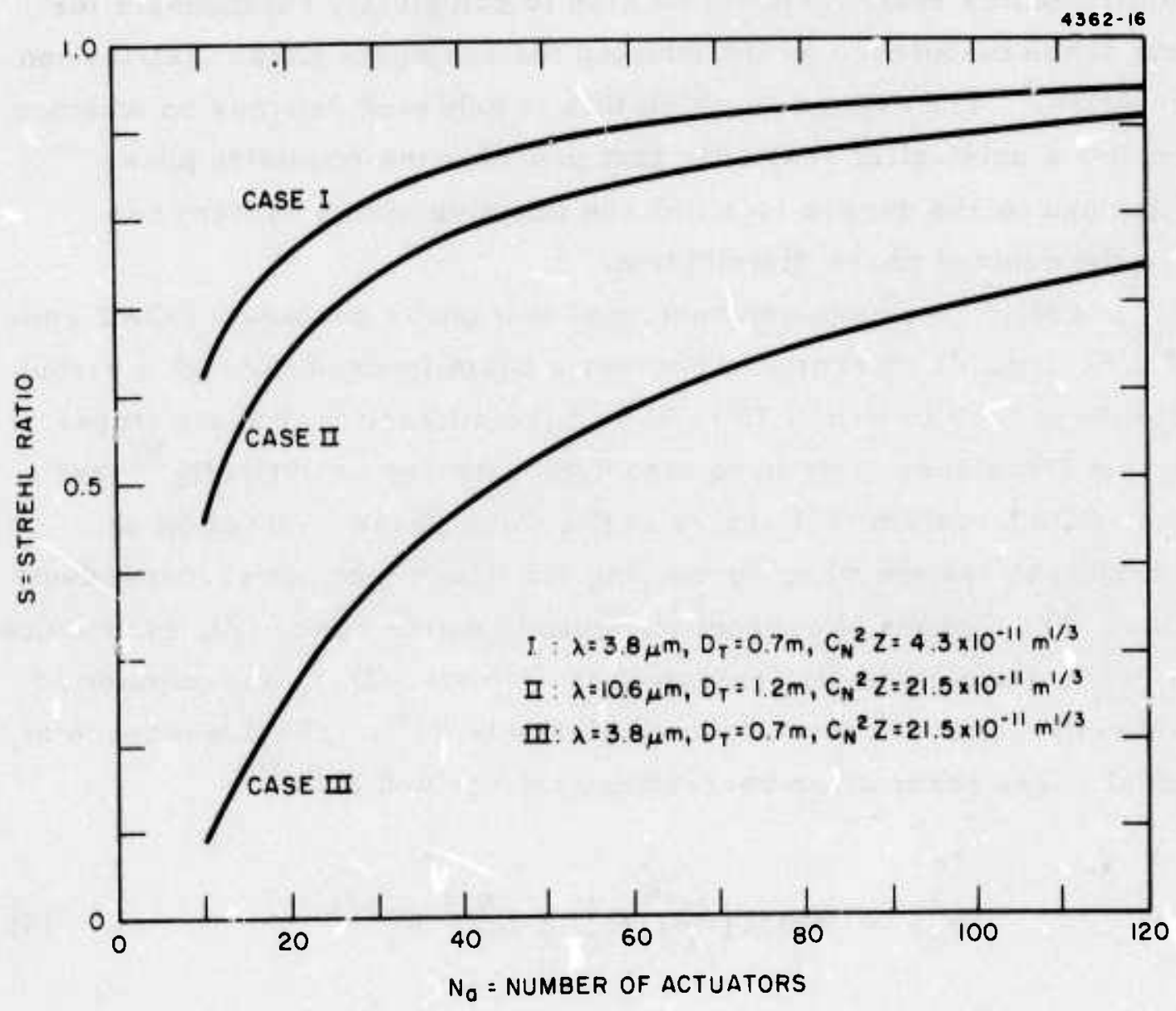

Fig. 29. Strehl ratio associated with atmospheric turbulence versis number of mirror actuators. 
To provide a check on the above analytical work, we have performed two types of mirror simulations. In each, the effect of a turbulent phase screen placed in front of an aperture was compensated by a mirror with a finite number of actuators. The average Strehl ratio obtained from a sequence of five independent phase screens was determined as a function of the number of actuators. In one of the simulations the deformable mirror software was used in conjunction with a sinusoidal multidither COAT algorithm of the type used previously at HRL in our COAT servo system studies. In the other simulation, the mirror was modeled by a segmented mirror with piston and tilt control on each segment. The piston and tilt settings for each segment were determined by a least squares fit to the random phase surface over the segment.

The agreement between the piston and tilt results and the theoretical predictions obtained from eqs. (5) and (6) is very good (see Ref. 30), which gives us confidence in the theoretical results given in these equations. We believe that these results are representative of those that would be obtained with a deformable mirror in the absence of the $2 \mathrm{~N} \pi$ problem is avoided.

The agreement between the theory and the deformarle mirrormultidicher COAT simulation results is reasonably good for moderate values of $\mathrm{C}_{\mathrm{N}}^{2} \mathrm{Z}$ but is poor for large values of $\mathrm{C}_{\mathrm{N}}^{2} \mathrm{Z}$. We attribute the poor results obtained at large values of $\mathrm{C}_{\mathrm{N}}^{2} \mathrm{Z}$ to a $2 \mathrm{~N} \pi$-type of behavior. A similar problem is observed with the focus control computer simulation. The deformable mirror simulation that was used in the se runs has a $2 \mathrm{~N} \pi$ correction loop that introduces a $2 \pi$ correction whenever the phase difference between actuators exceeds four radians. The intent is to suppress $2 \pi$ errors introduced by the servo system. However, if the phase distortion that is to be removed by the mirror changes by more than four radians between actuators, the $2 \pi$ "correction" introduced by the $2 \mathrm{~N} \pi$ correction loop is, in fact, a $2 \pi$ error and the mirror performance is corresponding degraded. This problem could be avoided by removing the $2 \mathrm{~N} \pi$ correction loop but then we would be faced with $2 \mathrm{~N} \pi$ servo errors. One way to avoid this problem is to use more actuators so that the phase change between actuators never 


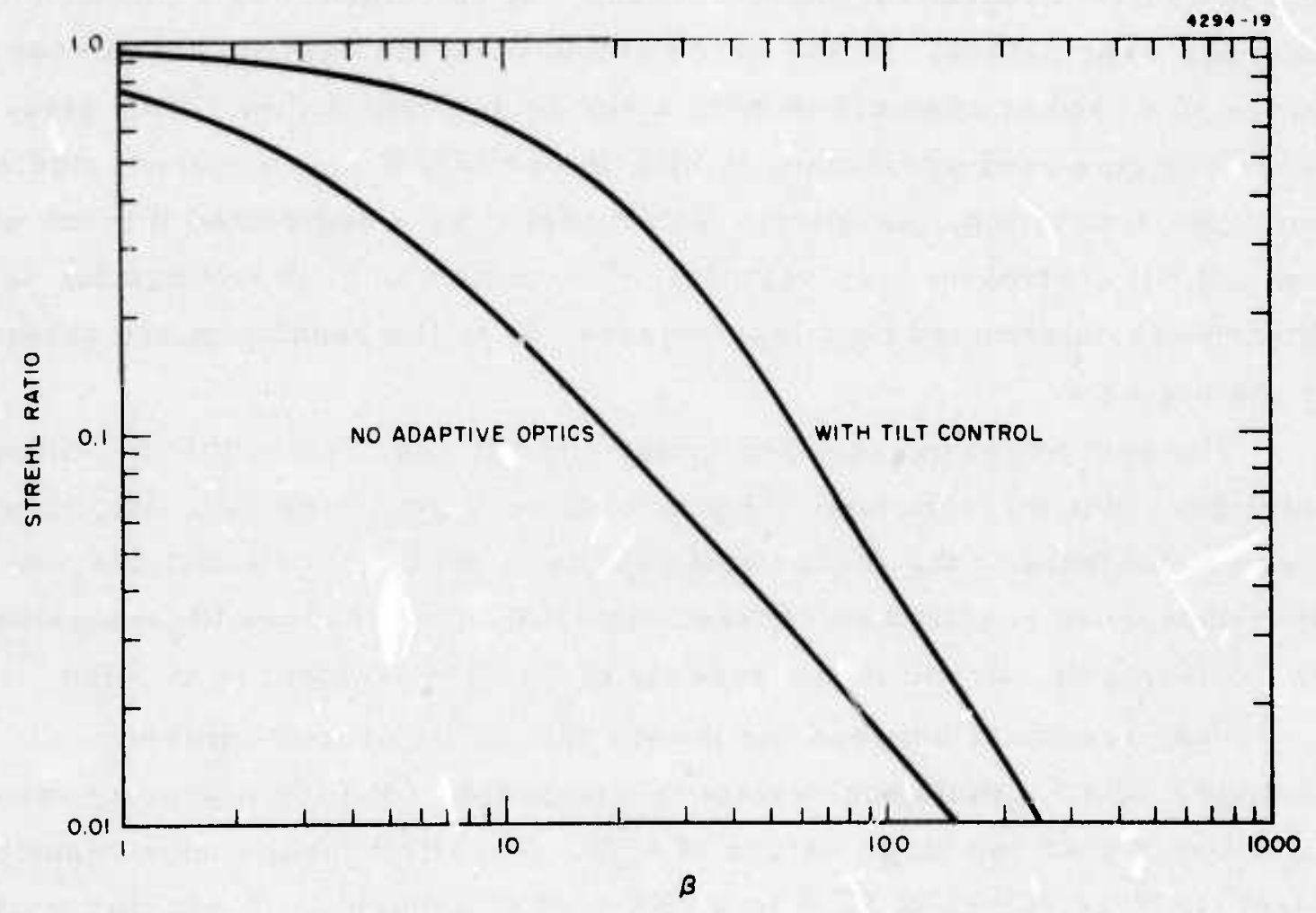

Fig. 30. Strehl ratio dut io Kolmogorov turbulence. 


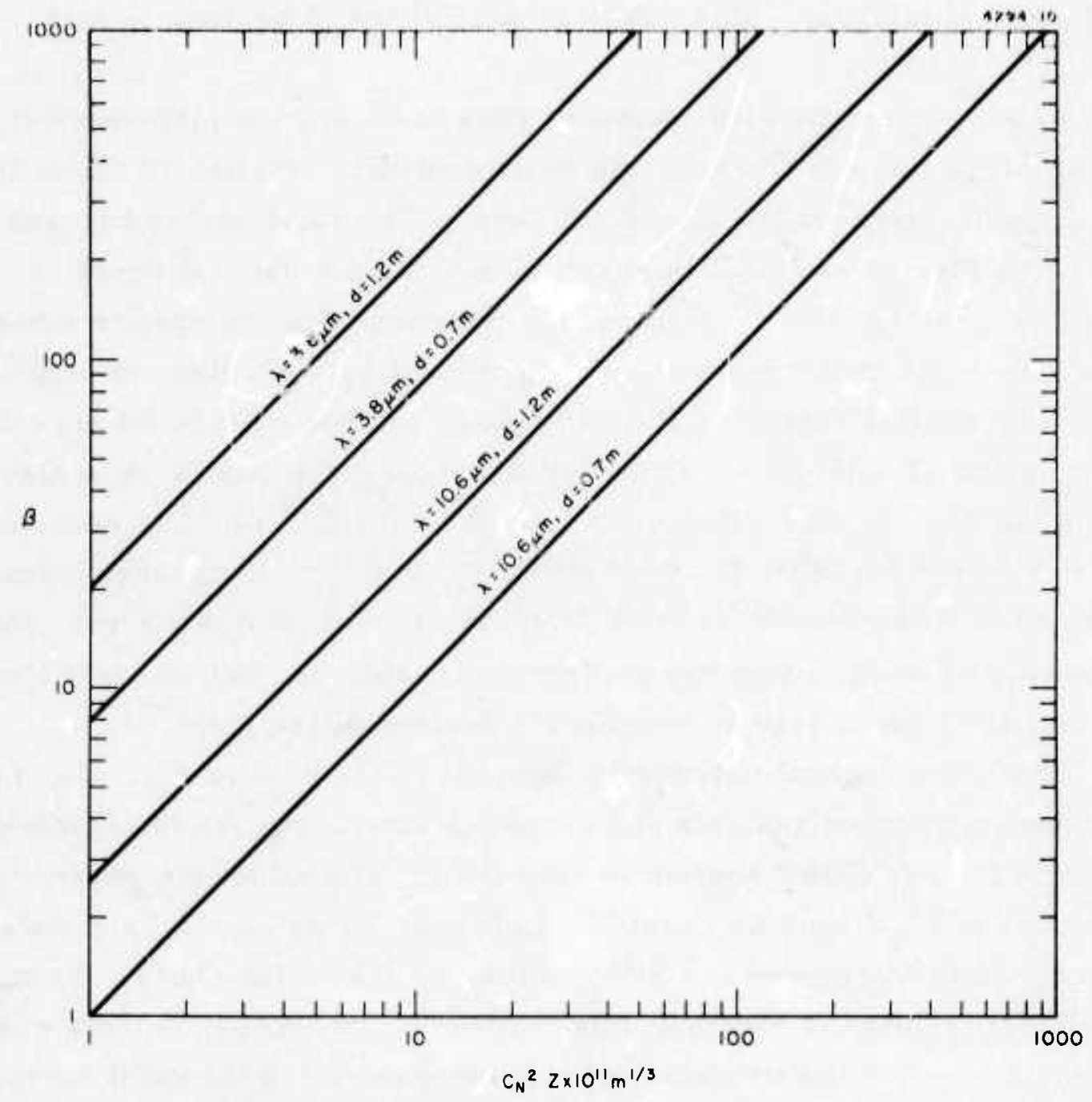

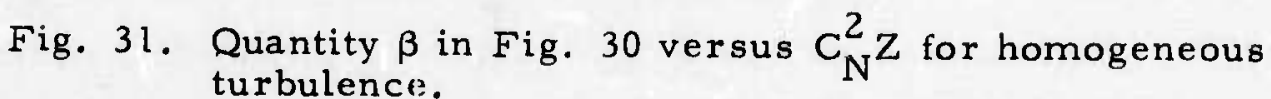


exceeds four radians. Another way would be to design the mirror so that the likelihood of $2 N_{\pi}$ errors is reduced, in which case the $2 \mathrm{N \pi}$ correction loop could be eliminated. More work clearly needs to be done on this problem.

We have made several computer runs to determine turbulence compensation using only the tracking and focus controls. Figure 30 shows the observed performance with tilt control alone. The relationship between the quantity $\beta$ in Fig. 30 and $C_{N}^{2} Z$ is shown in Fig. 31 for several cases of interest. Figure 32 shows a summary of the compensation results achieved with BAT controls compared with those produced by a 37 -element multidither COAT control system. The deformable mirror used in all the simulation runs has 37 actuators. The assumed propagation scenario is also shown in the figure. For reference, $\mathrm{C}_{\mathrm{N}}^{2} \mathrm{Z} \geq 21 \times 10^{-11} \mathrm{~m}^{1 / 3} \mathrm{i}$ s considered to be very ztrong turbulence. Also shown in the figure is the theorecical limit given by eqs. (5) and (6) for a 37 -element, deformable mirror, phaseconjugate COAT system that has perfect phase-sensing, but which is limited by the ability of the mirror to provide th: desired phase front.

There are several noteworthy features of the data in Fig. 32. First, the agreement between analysis and computer simulation for the 37-element deformable mirror COAT system is very good. The slight discrepancy at large values of $\mathrm{C}_{N}^{2} \mathrm{Z}$ may be caused by improper operation of the simulation routine designed to remove $2 n \pi$ ambiguities, as discussed above. Second, significant correction is obtained with the tracking or focus controls when used separately, but the two-axis focus control provides the most correction. In fact, when the tracking controls are combined with the two-axis focus control, little increase in Strehl ratio is produced over that observed with the focus control alone. We attribute the lack of a significant tilt component in the simulation (and thus little correction with the iracking control) to our assumption of an infinite outer scale for the turbulence. If we had used a finite outer scale, we expect that the tracking correction would have been more significant.

The significantly worse performance of a spherical* focus control above $C_{N}^{2} \mathrm{Z}=40 \times 10^{-11} \mathrm{~m}^{1 / 3}$ is also interesting. Finally, not unexpectedly,

\footnotetext{
* Two-axis focus control with both axes driven by the same dither and correction signals.
} 


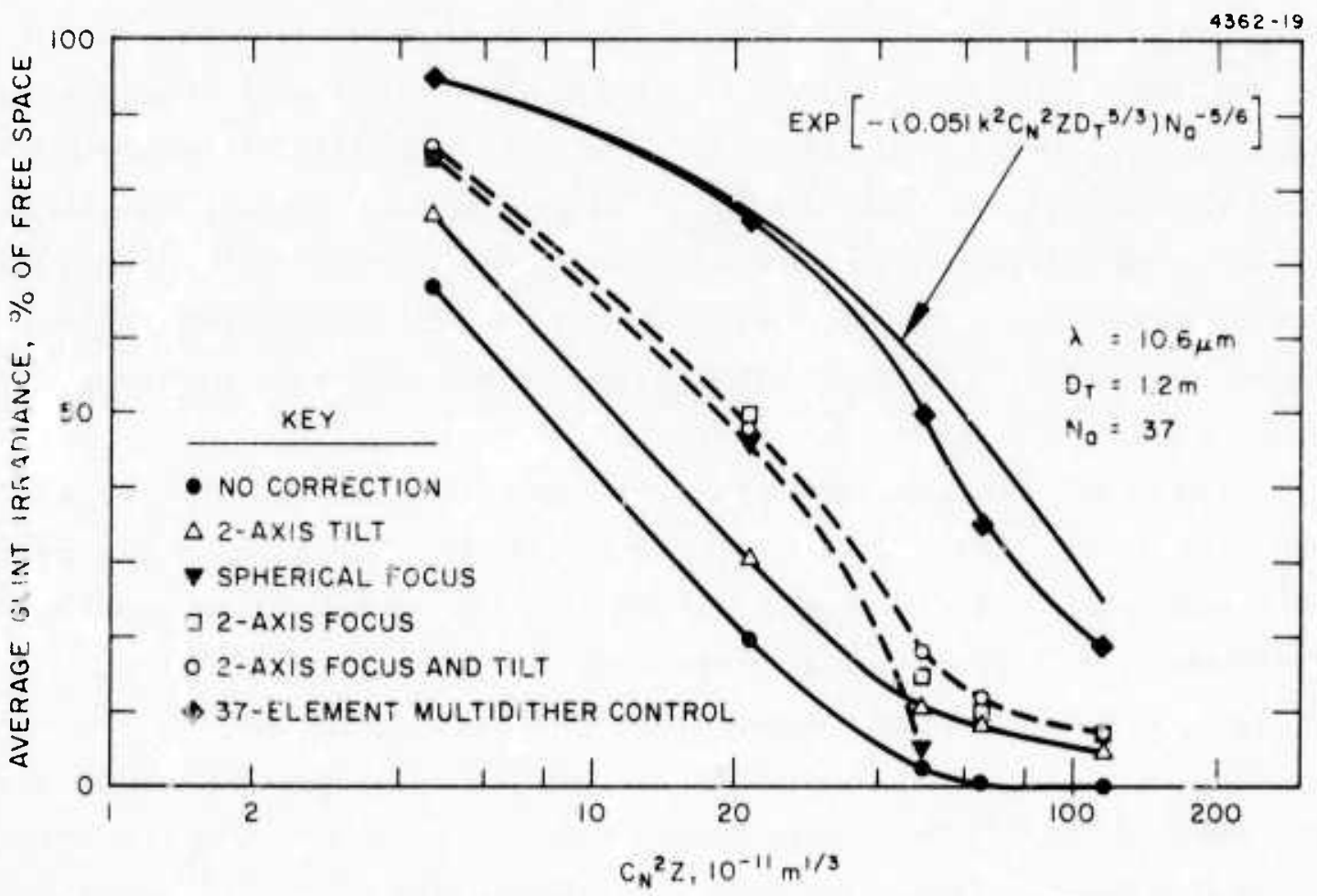

Fig. 32. Turbvlence compensation produced by computer simulation using 2".element deformable mirro:. Muitidither COAT contro? is compared to BAT (tracking and focus control, focus control only (both spherical and 2 -axis), and tracking control only. An infinite turbulence outer scale is used here, in contrast to Fig. 30 . 
a 37-channel multidither COAT system produces significantly more correction than the $\mathrm{BAT}$ controls alone; the $\mathrm{BAT}$ controls produce roughl; $\mathrm{a}$ factor of 2 improvement in Strehl ratio and the 37-channel COAT system can improve the Strehl ratio another factor of 2 .

a. Turbulence and Blooming - As we reported earlier, 8 the elfects of turbulence and thermal blooming can be considered separately, and then combined in a mean-square sum fashion to obtain the beam Strehl ratio when both effects are considered simultaneously. With this result, it is no: too surprising that COAT compensation for turbulence is unaffected by the presence of thermal blooming. The experimental data presented in Section III demonstrate this conclusion. Our coinputer simulations also confirm that good turbulence compensation is possible even if little or no blooming compensation occurs. Two examples of the simulation results are shown in Figs. 33 and 34. The transmitted beam in this case is a gaussian, truncated at the $10 \%$ intensity radius.

There are two principal observations to be made on the data in Figs. 33 and 34. First, there is very little thermal blooning compensation. This result is not consistent with our experimental work nor with other experimental ${ }^{16,17}$ or computer simulation ${ }^{19}$ work. We feel that the absence of correction in our simulations is caused by (1) the use of a returnwave correction algorithm and (2) by an isoplanatic problem associated with finite-width glints. ${ }^{30} \mathrm{O}, \mathrm{r}$ experimental data were taken with a fast outgoingvave multidither system. The data obtained by others $16,17,19$ were also produced by outgoing-wave systems, but in each case, the system was effectively a slow dicher system.

We are beginning to accumulate evidence that the inability of a phase conjugate system to correct for biooming - and its tendency to decrease the target irradiance with strong blooming - may be common to all "returnwave" COAT systems." A return-wave COAT system is one that operates only on the wavefront returned from the target to derive the servo

\footnotetext{
*We have observed, as have others, ${ }^{27}$ that for transmitter powers exceed ing the optimum power for blooming, a phase conjugate COAT correction is worse than doing nothing at all.
} 


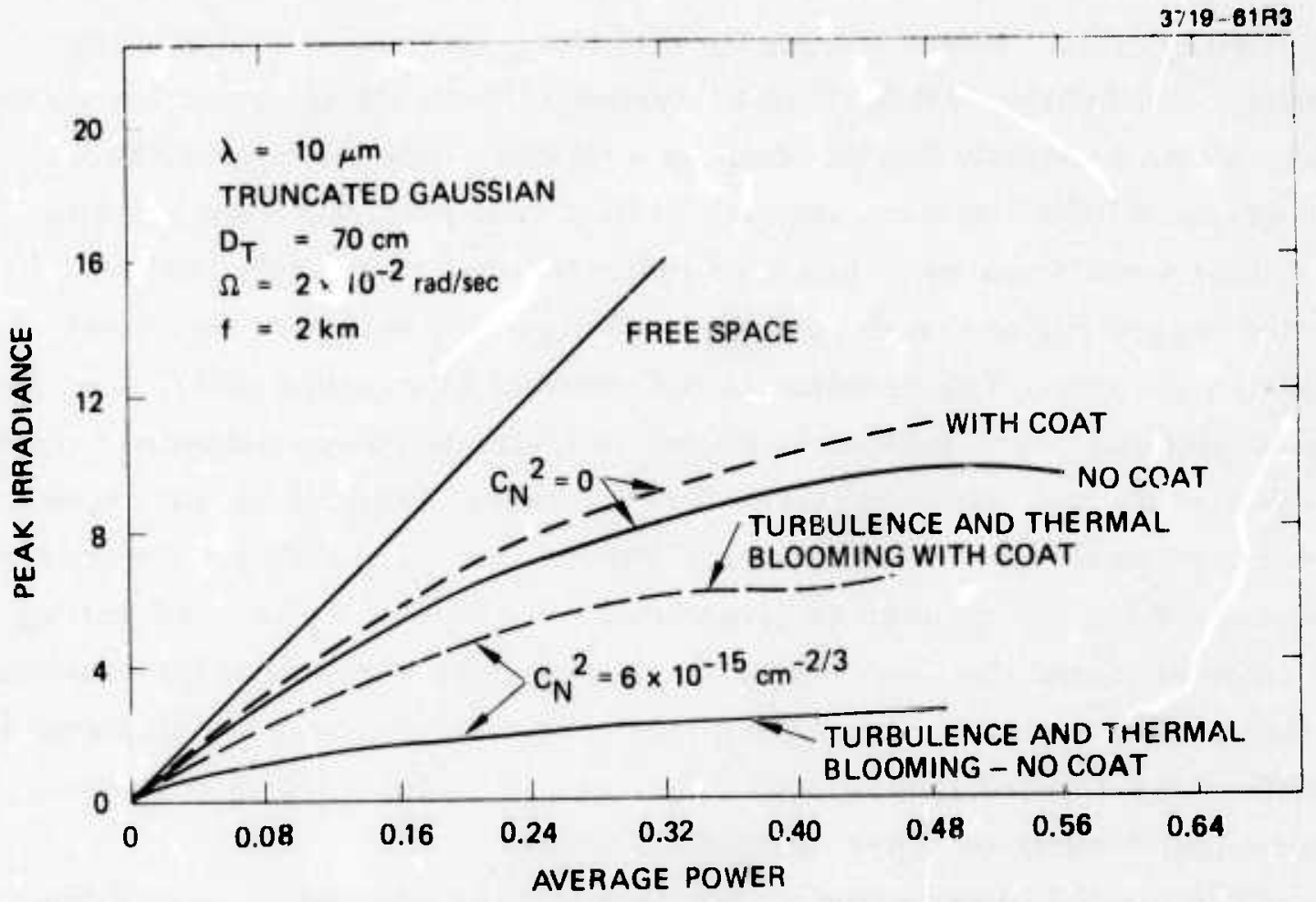

Fig. 33. Phase conjugate COAT correction for thermal blooming at $10.6 \mu \mathrm{m}$ for a truncated gaussian beam with beam slewing. Cases with and without turbulence are shown.

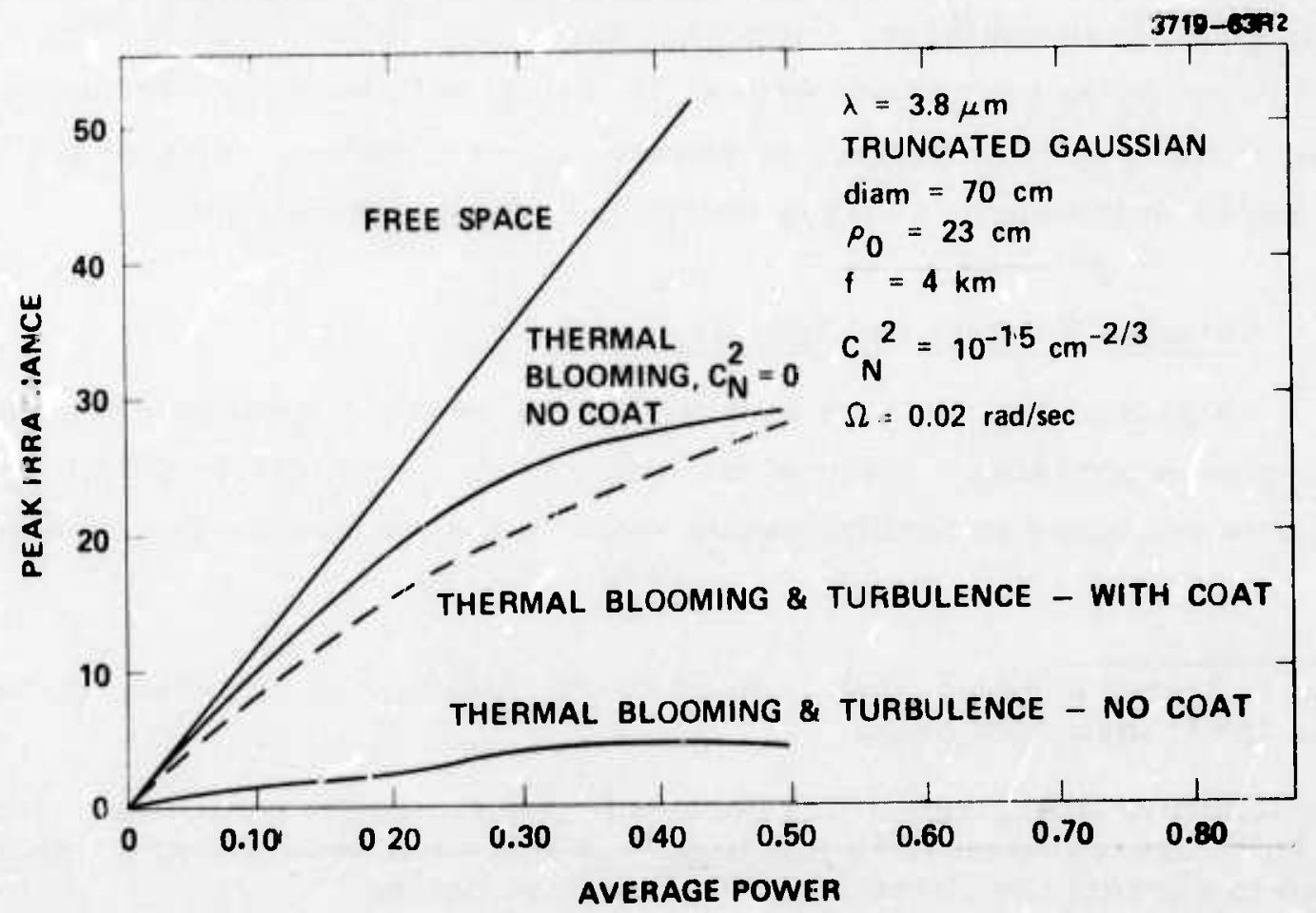

Fig. 34. Phase conjugate COAT correction for thermal blooming and turbulence at $3.8 \mu \mathrm{m}$ for a truncated gaussian beam with beam slewing. 
correction signals. Phase-conjugate systems, return-wave mullidither systems," and hybrid "TRIM-COAT" systems"*** a re all return-wave systems. In fact, of the presently known adaptive systems, only the multidither, outgoing-wave COAT system appears to be a true maximization system.

Our simulation work has also indicated that referencing off of extended target regions in the presence of blooming will give incorrect correction results. The problem is the thermal blooming equivalent of the finite isoplanatic patch size encountered in trying to image extended targets through turbulence. If the target reference region exceeds an atmospheric coherence diameter, $\dagger$ a COAT system will not be able to obtain the correct phase errors for the focused propigation path. We have observed during our computer simulation work with phase conjugate correction for blooming that this problern occurs even with glints less than one-half the diameter of the diffraction-limited transmitted beam on the target. We plan to study this problem further on other related programs.

The second observation on the data in Figs. 33 and 34 is that the ability to compensate for the effects of turbulence is apparently not degraded by the presence of thermal blooming effects. The net improvement in beam quality with phase conjugate compensation is most impressive for the $3.8 \mu \mathrm{m}$ case in Fig. 34 and for cases even at $10.6 \mu \mathrm{m}$, with large transmitter apertures. This is to be expected, of course, since turbulence effects are most pronounced in the cases of large beams and shorter wavelengths.

B. Complex Targets and Speckle Modulations

As part of the contract redirection defined by Amendment No. 2 , we have been pursuing a study of the behavior of a multidither COAT system when it is subjected to multiplicative noise in the receiver. The type of

"The reflected coherent light sensed by the receiver is dithered, rather than the transmitted beam.

*** Transmitting-Imaging: broad band, incoherent target returns are sensed $\overline{b y}$ an image-compensating Imaging-COAT system and this information is used to correct the phase of the transmitted beam.

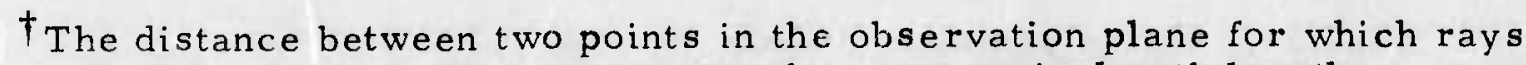
emanating from a point source see the same optical path length. 
noise considered is very similar to the modulations produced by complextarget speckle effects (see Section III and Ref. 29). Our analytical studies have utilized both closed form analysis and computer simulation of an 18-channel multidither servo.

1. Analytical Models for Speckle and COAT Servo

In the previous quarterly report, ${ }^{6}$ we developed a statistical analysis intended to predict and explain the behavior of a COAT system subjected to multiplicative speckle modulations. This analysis will now be used to calculate expectation values of various COAT system parameters. We then compare these calculations to data gathered from computer simulations.

The first parameters of interest are $P_{D}$, the average power of the dither modulation signal, and $\mathrm{P}_{S}$, the average power of the speckle signal within $\pm 400 \mathrm{~Hz}$ of any dither frequency. The expression derived in Ref. 6 for $P_{D}$ for $N$ servo channels is

$$
P_{D}=\frac{K^{2}}{8}\left(\frac{4 J_{o}(\psi) J_{1}(\psi)}{N^{2}}\right)^{2} \sum_{n=1}^{N} b_{n}^{2} \text {, }
$$

where $\mathrm{K}$ is a receiver proportionality constant, $\psi$ is the dither amplitude, and

$$
b_{n} \equiv \sum_{\substack{m=1 \\ n \neq m}}^{N} \sin \left(\beta_{n}-\beta_{m}\right)
$$

The $J_{0}(\psi)$ and $J_{1}(\psi)$ functions in eq. (7) are the zero- and first-order Bessel functions and $\beta_{i}$ is the phase error signal in the $i^{\text {th }}$ control channel. The expectation value of $P_{D}$ is 


$$
\left\langle\mathrm{P}_{\mathrm{D}}\right\rangle=\frac{\mathrm{K}^{2}}{8}\left(\frac{4 \mathrm{~J}_{0}(\psi) \mathrm{J}_{1}(\psi)}{\mathrm{N}^{2}}\right)^{2} \sum_{\mathrm{n}-1}^{\mathrm{N}}\left\langle\mathrm{b}_{\mathrm{n}}^{2}\right\rangle
$$

Sirce $b_{n}$ is a functio: of the random variables $\beta_{1}, \beta_{2}, \ldots, \beta_{N}$, it is also a random variable whose expectation value is expressed as

$$
\left\langle b_{n}^{2}\right\rangle=\int d \beta_{1} \int d \beta_{2} \cdots \int d \beta_{N} P\left(\beta_{1}\right) P\left(\beta_{2}\right) \cdots P\left(\beta_{N}\right) b_{n}^{2}\left(\beta_{1}, \beta_{2} \cdots \beta_{N}\right)
$$

where $P\left(\beta_{i}\right)$ is the probability density of $\beta_{i}$.

We assume that the $\beta_{i}$ 's are uniformly distributed between the phase angles $\alpha_{0}$ and $\alpha_{0}+\alpha$ as indicated in Fig. 35. Since $\alpha_{0}$ is arbitrary, it is convenient to define it as a $\alpha_{0} \equiv \alpha / 2$; this will make the integrals in eq. (9) easier to calculate. Physically, $\alpha$ is the instantaneous mean phase error, which the COAT system is trying to drive to zero. With this definition, as $\alpha$ approaches zero, the COAT array converges. Conversely, as $\alpha$ approaches $2 \pi$, the array approaches a convergence level of zero.

For any given $\beta_{i}$, the probability density is $P\left(\beta_{i}\right)=\alpha^{-1}$ over a region $-\alpha / 2<\alpha_{i}<\alpha / 2$, and zero elsewhere. Using the above information plus the definition of $b_{n}$ in eq. (7), we can write eq. (9)

$$
\begin{aligned}
\left\langle b_{n}^{2}\right\rangle= & \alpha^{-3} \sum_{\substack{m=1 \\
m \neq k, n \\
k \neq n}}^{N} \sum_{k=1}^{N} \int_{-\alpha / 2}^{\alpha / 2} d \beta_{m} \int_{-\alpha / 2}^{\alpha / 2} d \beta_{K} \int_{-\alpha / 2}^{\alpha / 2} d \beta_{n} \sin \left(\beta_{n}-\beta_{m}\right) \sin \left(\beta_{n}-\beta_{k}\right) \\
& +\alpha^{-2} \sum_{\substack{m=1 \\
m \neq n}}^{N} \int_{-\alpha / 2}^{\alpha / 2} d \beta_{m} \int_{-\alpha / 2}^{\alpha / 2} d \beta_{n} \sin ^{2}\left(\beta_{n}-\beta_{m}\right)
\end{aligned}
$$




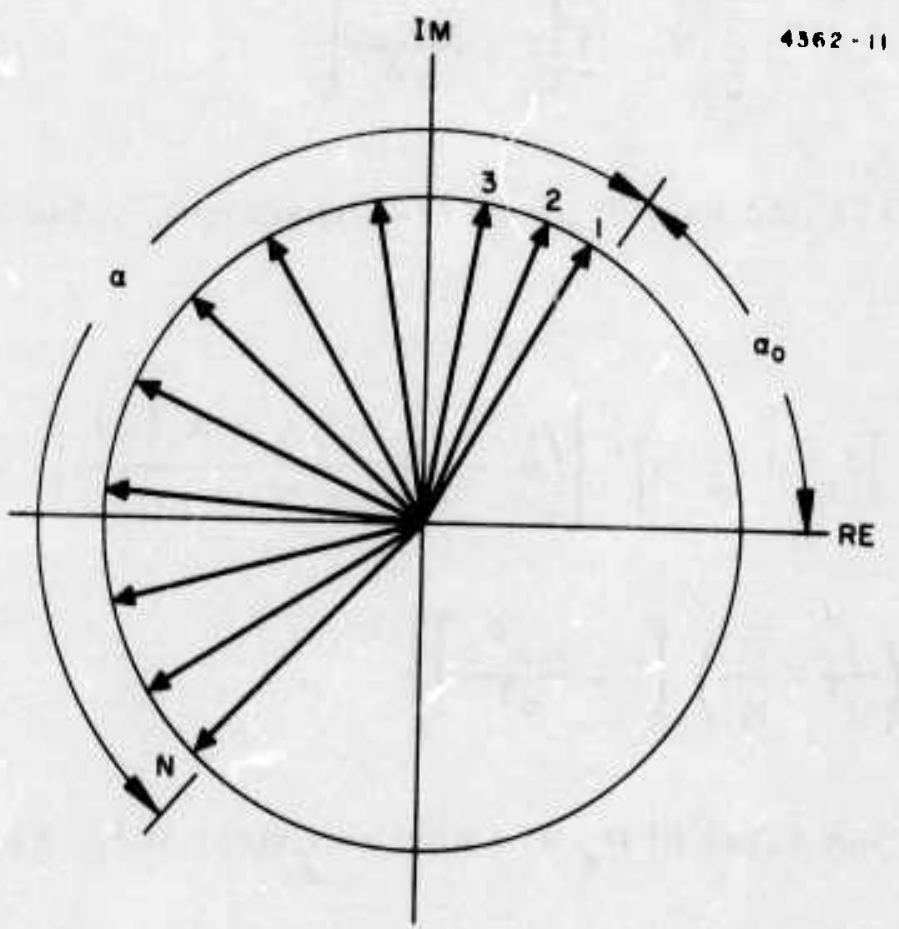

Fig. 35. Phaser diagram of a partially converged, $\mathrm{N}$-element COAT a rray defining the quantities $\alpha$ and $\alpha_{0}$ used in the text. 
Carrying out the integrals and summations in eq. (10) gives

$$
\begin{aligned}
\left\langle b_{n}^{2}\right\rangle= & \frac{1}{2}\left(N^{2}-3 N+2\right) \frac{\sin ^{2}\left(\frac{\alpha}{2}\right)}{(\alpha / 2)^{2}}\left[1-\frac{\sin \alpha}{\alpha}\right] \\
& +\frac{1}{2}(N-1)\left[1-\frac{\sin ^{2}}{\alpha^{2}}-\right]
\end{aligned}
$$

Substitution of eq. (11) into eq. (8) gives the expectation value of the average dither pwer:

$$
\begin{aligned}
<\mathrm{P}_{\mathrm{D}}= & \mathrm{K}^{2}\left[\mathrm{~J}_{0}(\psi) \mathrm{J}_{1}(\psi)\right]^{2}\left\{\left(\frac{1}{\mathrm{~N}}-\frac{3}{\mathrm{~N}^{2}}+\frac{2}{\mathrm{~N}^{3}}\right) \frac{\sin ^{2}\left(\frac{\alpha}{2}\right)}{(\alpha / 2)^{2}}\left[1-\frac{\sin _{\alpha} \alpha}{\alpha}\right]\right. \\
& \left.+\left(\frac{1}{\mathrm{~N}^{2}}-\frac{1}{\mathrm{~N}^{3}}\right)\left[1-\frac{\sin ^{2} \alpha}{\alpha^{2}}\right]\right\}
\end{aligned}
$$
viously, 6

The expectation value of $\mathrm{P}_{\mathrm{s}}$ will now be calculated. As derived pre-

$$
P_{s} \equiv \frac{1}{2} K^{2} I_{M}^{2} \sum_{k=1}^{N} \sum_{j=1}^{M} a_{j k}^{2}
$$

where $I_{m}$ is the mean convergence level and $a_{j} k$ is a random amplitude variable that characterizes the amplitude of the spurious modulation at frequency $\omega_{\mathrm{jk}}$. We have assumed that the re are $\mathrm{M}$ discrete modulation frequencies a round each of the $\mathrm{N}$ cither frequencies so that the spurious modulation has the form 


$$
M_{s}=\frac{1}{2}+\sum_{k=1}^{N} \sum_{j=1}^{M} a_{j k}\left(\omega_{j k} t+\phi_{j k}\right)
$$

where $\phi_{j} k$ is a randcmly chosen phase between zero and $2 \pi$.

Since $I_{M}^{2}$ is a function of the independent random variables $\beta_{1}$,

$\beta_{2}, \cdots \beta_{N}$ and the $a_{j k}$ are themselves independent random variables, the expectation value of $\mathrm{P}_{\mathrm{s}}$ can be expressed as

$$
\left\langle P_{s}>=\frac{1}{2} K^{2}<I_{M}^{2}>\sum_{k=1}^{N} \sum_{j=1}^{M}<a_{j k}^{2}>\right.
$$

In our computer simulations, the $a_{j k}$ were chosen randomly between the values of 0 and $a_{\max }$. The probability distribution is this uniform over that interval and zero elsewhere. The expectation value of their square is

$$
\left\langle a_{j k}^{2}\right\rangle=\frac{1}{a_{\max }} \int_{0}^{a_{\max }} a^{2} d a=\frac{a_{\max }^{2}}{3} .
$$

The mean convergence level, $I_{M}$, is calculated ${ }^{6}$ to be

$$
I_{M} \equiv \frac{1}{N}+\frac{J_{0}^{2}(\psi)}{N^{2}} \sum_{n=1}^{N} \sum_{\substack{m \\ n \neq m}}^{N} \cos \left(\beta_{n}-\beta_{m}\right)
$$

The expectation value of $\mathrm{I}_{M}^{2}$ is

$$
\left\langle I_{M}^{2}\right\rangle=\int d \beta_{1} \int d \beta_{2} \cdots \int d \beta_{N} P\left(\beta_{1}\right) P\left(\beta_{2}\right) \cdots P\left(\beta_{N}\right) I_{M}^{2}\left(\beta_{1}, \beta_{2} \cdots \beta_{N}\right)
$$


Substituting the definition of $I_{M}$ given in eq. (17) and the probability densities $\left(P\left(\beta_{i}\right)=d^{-1}\right)$ into eq. (18), we can solve the expectation value of $I_{M}^{2}$. These steps will be omitted, however, for brevity. The result of the calculation is

$$
\begin{aligned}
\left\langle\mathrm{I}_{\mathrm{M}}^{2}>=\right. & \frac{1}{\mathrm{~N}^{2}}+2 \mathrm{~J}_{0}^{2}(\psi)\left(\frac{1}{\mathrm{~N}}-\frac{1}{\mathrm{~N}^{2}}\right) \frac{\sin ^{2}(\alpha / 2)}{(\alpha / 2)^{2}}+\mathrm{J}_{0}^{4}(\psi)\left\{\left(1-\frac{6}{\mathrm{~N}}+\frac{11}{\mathrm{~N}^{2}}-\frac{6}{\mathrm{~N}^{3}}\right) \frac{\sin ^{4}(\alpha / 2)}{(\alpha / 2)^{4}}\right. \\
& +2\left(\frac{1}{N}-\frac{3}{\mathrm{~N}^{2}}+\frac{2}{\mathrm{~N}^{3}}\right) \frac{\sin ^{2}(\alpha / 2)}{(\alpha / 2)^{2}}\left[1-\frac{\sin \alpha}{\alpha}\right] \\
& \left.+\left(\frac{1}{N^{2}}-\frac{1}{N^{3}}\right)\left[1-\frac{\sin ^{2} \alpha}{\alpha^{2}}\right]\right\}
\end{aligned}
$$

Figure 36 plots the root-mean-square value of $I_{m}$ found from eq. (19) as a fraction of $\alpha$ for an 18-channel COAT system. When eqs. (16) and (19) are substituted into eq. (15), the expectation value of the average power in the speckle modulation signal is found to be

$$
\begin{aligned}
\left\langle\mathrm{P}_{\mathrm{s}}\right\rangle= & \frac{1}{6} \mathrm{~K}^{2}(\mathrm{NM}) \mathrm{a}_{\max }^{2}\left\{\frac{1}{\mathrm{~N}^{2}}+2 \mathrm{~J}_{0}^{2}(\psi)\left(\frac{1}{\mathrm{~N}}-\frac{1}{\mathrm{~N}^{2}}\right) \frac{\sin ^{2}(\alpha / 2)}{(\alpha / 2)^{2}}\right. \\
& +\mathrm{J}_{0}^{4}(\psi)\left[\left(1-\frac{6}{\mathrm{~N}}+\frac{11}{\mathrm{~N}^{2}}-\frac{6}{\mathrm{~N}^{3}}\right) \frac{\sin ^{4}(\alpha / 2)}{(\alpha / 2)}\right. \\
& +2\left(\frac{1}{N}-\frac{3}{\mathrm{~N}^{2}}+\frac{2}{\mathrm{~N}^{3}}\right) \frac{\sin ^{2}(\alpha / 2)}{(\alpha / 2)^{2}}\left(1-\frac{\sin \alpha}{\alpha}\right) \\
& \left.\left.+\left(\frac{1}{N^{2}}-\frac{1}{N^{3}}\right)\left(1-\frac{\sin ^{2} \alpha}{\alpha^{2}}\right)\right]\right\}
\end{aligned}
$$

Expectation values of many other parameters can be calculated in a similar manner. Each calculation will give expressions that are functions of 


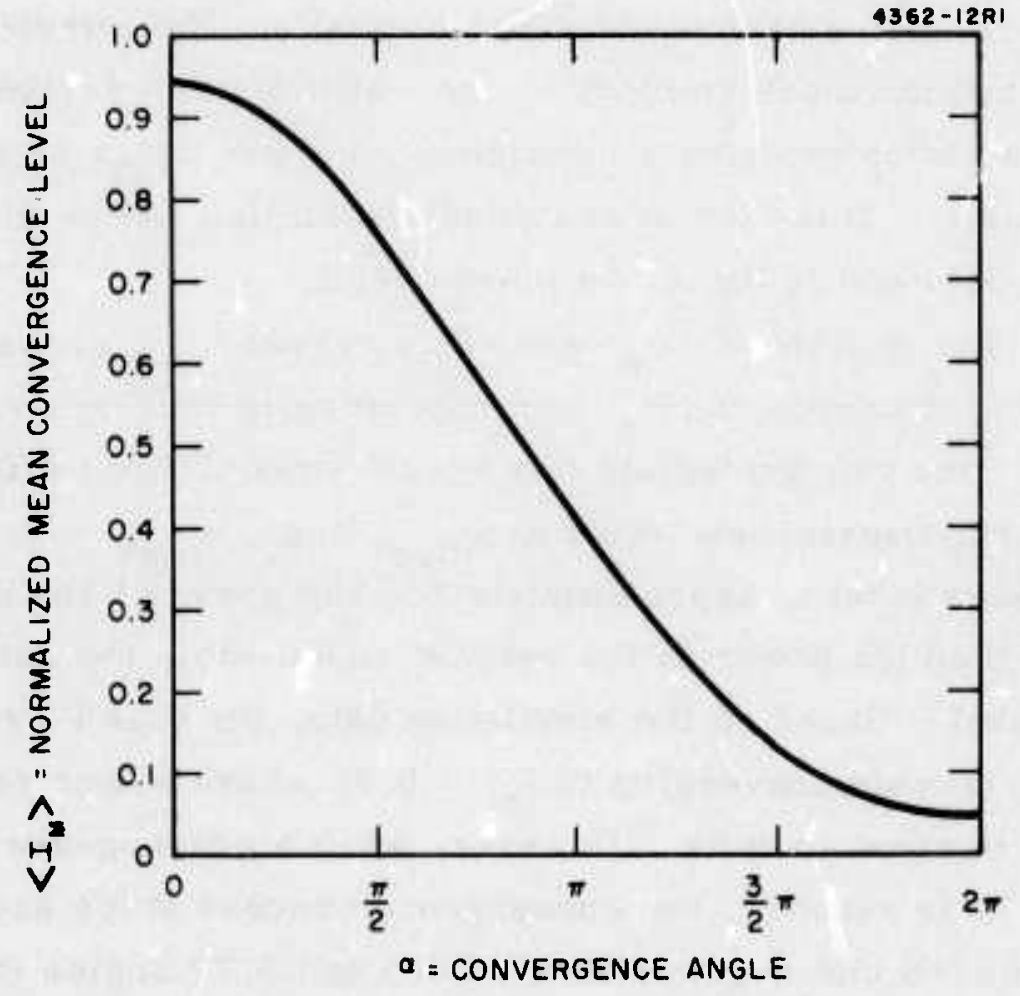

Fig. 36. Statistical expectation value of the mean convergence level, $I_{M}$, for an 18 -channel multidither COAT system (dither amplitude $=20^{\circ}$ ), calculated from eq. (13). 
$\mathrm{N}, \psi$, and $\alpha$ and possibly other variables such as a max $\mathrm{K}$, etc., which are included in the definitions of a given parameter. For any particular COAT system, most of these variables will be specified. The variable $\alpha$, how ever, is a statistical parameter which characterizes the convergence level based on an irtuitive model. It is therefore questionable whether one could ever measure such a parameter experimentally. Nevertheless, krowing several expectation values as functions of allows us to derive expressions for one expectation value as a function of another: $\left\langle\mathrm{P}_{D}\right\rangle$ as a function of $\left\langle\mathrm{I}_{\mathrm{M}}\right\rangle$, for example. This kind of analytical prediction can be checked against the real world and is therefore more useful.

Figure 37 shows plots of $\left\langle\mathrm{P}_{\mathbf{S}}\right\rangle$ and $\left\langle\mathrm{P}_{\mathrm{D}}\right\rangle$ versus $\left\langle\mathrm{I}_{\mathrm{M}}\right\rangle$ calculated in this manner. Several curves of $\left\langle\mathrm{P}_{\mathrm{s}}\right\rangle$ corresponding to different values of $a_{\max }$ are shown. One can appreciate how speckle modulations efiect a COAT system by considering one val'ue of $a_{\text {max' }}$ e. g. , $a_{\max }=0.006$. Below a convergence level of approximately 0.5 the power in the dither signal is greater than the power in the speckle signal while the converse is true above this level. Based on the simulation data, the COAT system apparently has no trouble converging to $I_{M}=0.5$, where power ratio, $p=\left\langle P_{D}\right\rangle /\left\langle P_{S}\right\rangle$, is equal to unity. However, when a convergence level of approximately 1.77 is reachea, the convergence process stops and a random oscillation between the convergence level of 0.5 and 0.77 begins (the computer simulation runs discussed below exhibit this behavior).

From the data in Fig. $3 \%$ it is evident that for any given $a_{\text {max }}$ the ratio $R_{1}$, of the dither power to the speckle power varies over a large range of values as a function of convergence level. For the example of $a_{\max }=0.006$, the power ratio is very small at a convergence level of 0.77 (the actual value is about 0.3 ). With the speckle power so much larger than the dither power it is reasonable to expect convergence problems.

2. Computer Simulation Code

Along with this analysis, we have been making computer runs using the detailed COAT servo simulation shown in Fig. 38. The spurious modulation function $M_{s}$ (as defined by eq. (14)) in our work to date) 


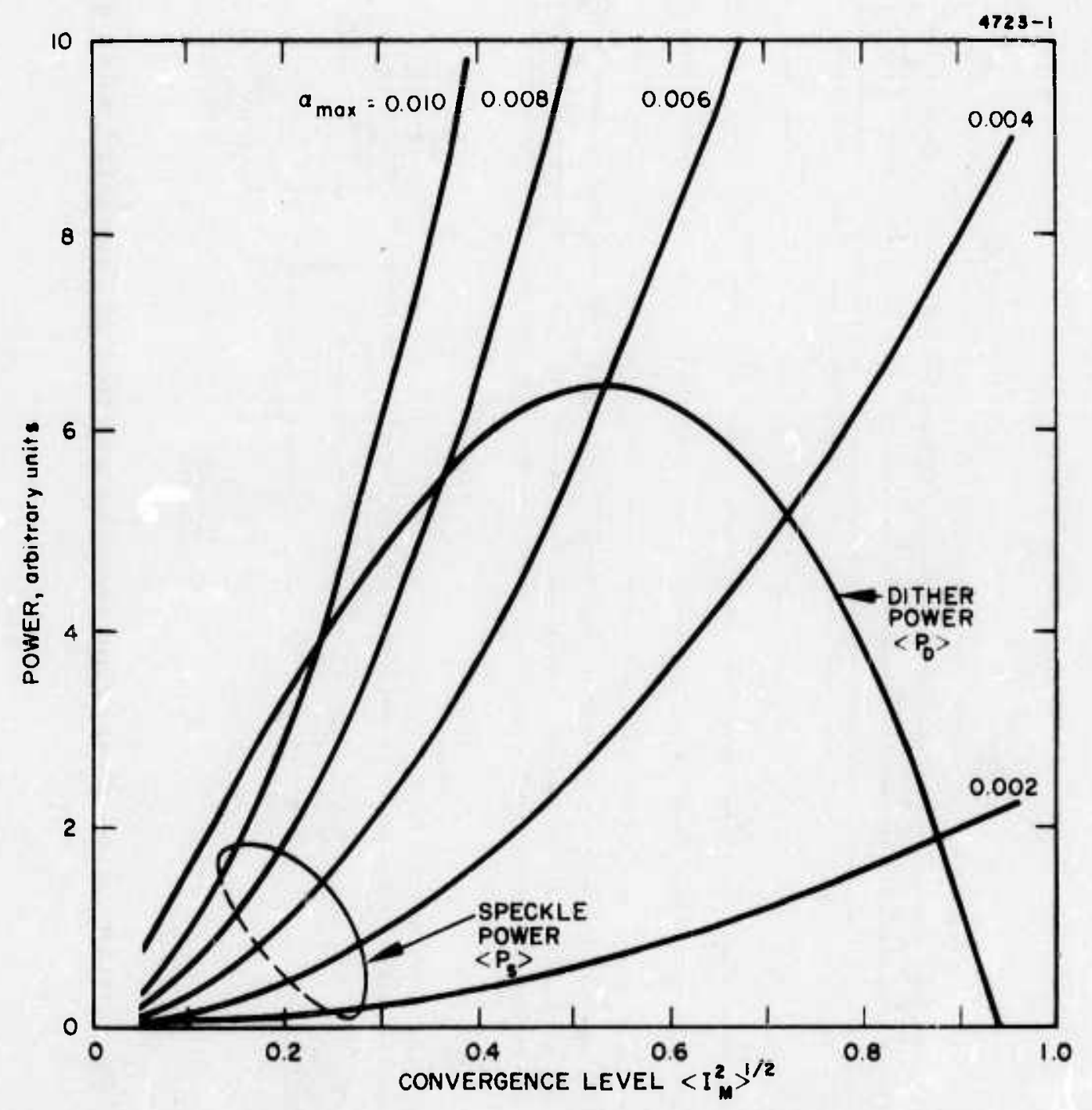

Fig. 37. Theoretical dither power and speckle power versus convergence level for an 18-element COAT array $\left(\psi=20^{\circ}\right)$. 


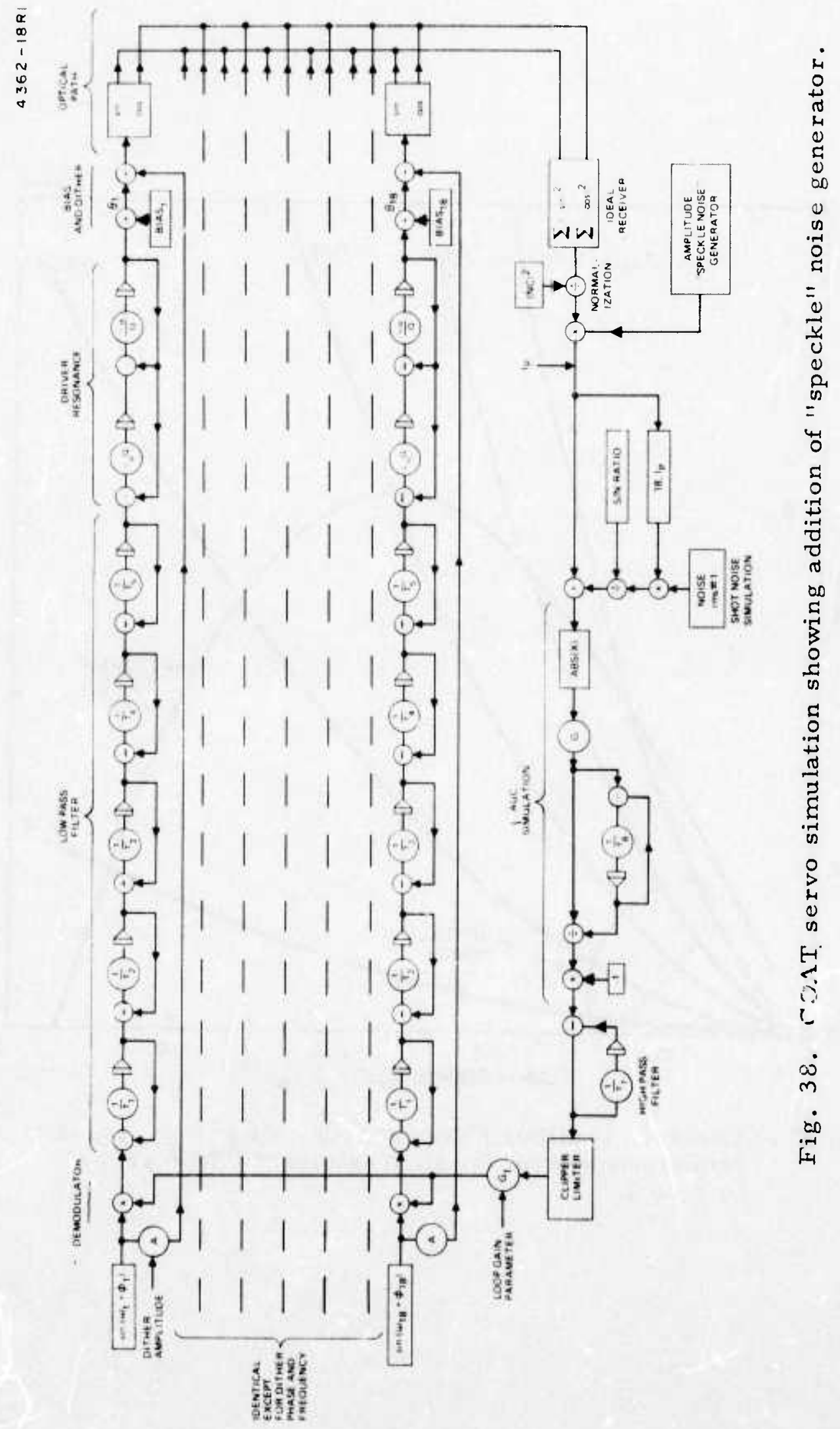


is introduced as multiplying the normal receive $r$ signal. For the simulation studies performed to date, we have chosen $M=21$ and spaced the se noise frequencies $\pm 83 \mathrm{~Hz}$ around each dither frequency. This is a rather arbitrary choice, but it does ensure that all spurious modulation is well within the $400 \mathrm{~Hz}$ servo bandwidth. An amplitude limitation was also included in the simulation to keep the return signal from exceeding an absolute value of unity just before entering the loop gain amplifier (noted as $G_{1}$ in Fig. 37). This modification, which avoids instabilities resulting from the automatic gain control, is used in the laboratory midel of the COAT array. Simulations we re run at three values of broad-band signal-to-noise ratio (receiver shot noise): $10^{10}, 40$, and 20 . When $(\mathrm{S} / \mathrm{N})_{\text {shot }}=10^{10}$ and $a_{\max }=0$ (no speckle noise), the simulation produces a time history of the power on the target glint as shown in Fig. 39(a). When the speckle noise modulation shown in Fig. $39(c)$ is present $\left(a_{\max }=0.008\right)$; the time history in Fig. $38(b)$ results. In this case, the rms value of $\mathrm{M}_{\mathrm{s}}$ is only 0.064 (around $\mathrm{M}_{\mathrm{s}}=0.50$ ), but the maximum peak-to-peak value is about 0.4 .

The behavior shown in Fig. $39(\mathrm{~b})$ for $\mathrm{a}_{\max } \neq 0$ is typical. In general, the speckle noise has two effects: (I) the maximum percent convergence level is lowered, and (2) a random oscillation of the intensity occurs between the new maximum convergence level and a minimum level. This oscillation is much slower than the dither oscillations and its magnitude varies with convergence level. Figure 40 shows a plot of the maxima and minima recorded during these simulations as a function of $a_{\text {max }}$. The open loop gain, $G_{1}$, was the same for each case. At high average convergence levels (low values of $a_{\text {max }}$ ), the random oscillations have much smaller magnitudes than at the low convergence levels (high values of $a_{\max }$ ).

\section{Analysis Compared with Computer Simulation}

When we examine other speckle power curves in Fig. 36 which correspond to different values of $a_{\text {max }}$ and cross check these with the computer simulation data, it becomes apparent that the power ratio is a very important parameter, defined as

$$
\rho \equiv \frac{\left\langle P_{D}\right\rangle}{\left\langle P_{S}\right\rangle}
$$



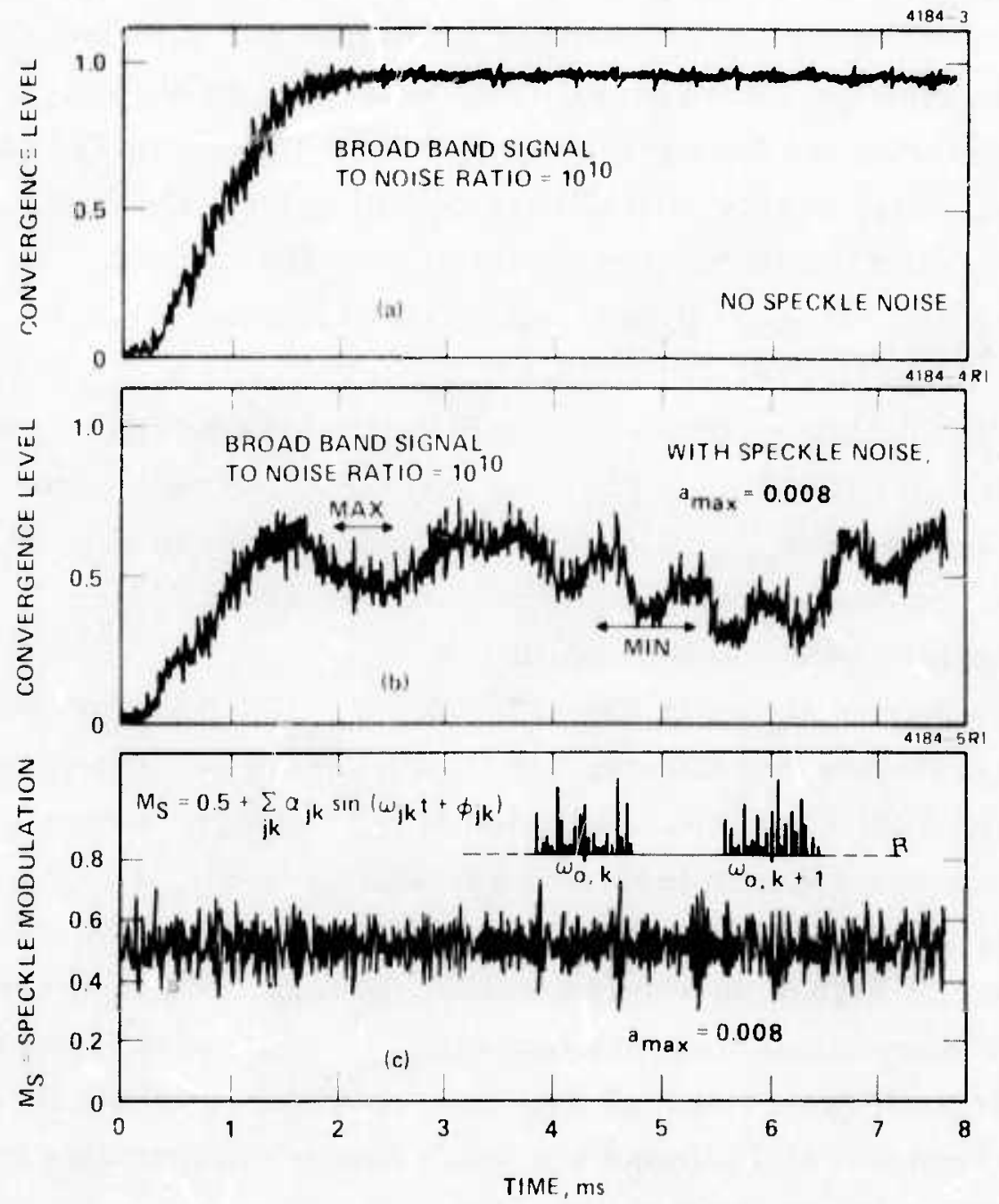

Fig. 39. COAT computer simulation time histories. (a) Broadband shot noise signal-to-noise ratio $=\left(\mathrm{S} / \mathrm{N}_{\text {shot }}=1010\right.$ and $\mathrm{a}_{\max }=0$ (no speckle noise). (b) a $\max =0.008$. (c) Speckle noise modulation used to produce (b). 
Then as the convergence process begins, the value of $\rho$ is much greater than 1. As the convergence level increases, the value of $\rho$ gets progressively smaller. For a given a max the COAT system should converge to the level corresponding to $\rho=1$ (where the $\left\langle\mathrm{P}_{\mathrm{s}}\right\rangle$ and $\left\langle\mathrm{P}_{\mathrm{D}}\right\rangle$ curves in Fig. 35 cross). The final convergence level corresponding to $\rho=1$ and that corresponding to some smaller value of $\rho$.

We can use the data in Fig. 40 along with the analysis developed above to verify our hypothesis that the convergence level oscillates between two fixed values of $\rho$ and to find these values. This is done by first choosing $\rho$ (e.g., $\rho=1$ ). Equation (12) is then used to calculate $\left\langle P_{D}\right\rangle$ as a function of $\alpha$ (or equivalently, $\left\langle\mathrm{I}_{\mathrm{m}}^{2}\right\rangle^{1 / 2}$ ). Equations (20) and (21) are then used to calculate $a_{\max }$ as a function of $\left\langle\mathrm{I}_{\mathrm{m}}^{2}\right\rangle^{1 / 2}$. Using this method, we have found that $\rho_{\max }=1.0$ and $\rho_{\text {min }}=0.3$ correspond to the maximum and minimum convergence levels. Figure 41 compares the results of our analytical calculations with the computer-generated data of Fig. 39. The agreement between the analytical theory and the simulation data is excellent for signalto-noise (shot noise) ratios, $S / N$, between $10^{10}$ and 40 . The agreement is not as good for the $S / n=20$ case, but this is not unexpected since the computer simulations have always shown degradation in COAT system performance when $\mathrm{S} / \mathrm{N}$ was chosen less than 40 , with or without speckle modulations. These data thus appear to verify the original hypothesis that motivated much of the statistical analysis, namely that the maximum and minimum convergence levels correspond to constant values of the dither-to-speckle power ratio.

The good agreement between our simplified analysis and the computer simulation data has given us confidence that we can accurately predict the performance of a multidither COAT system in the presence of spurious modulations. Our work to date has been with a particular COAT system and a hypothetical model for the spurious modulations (chosen, however, to present maximum difficulty to the COAT system). We expect that the analysis can be extended in a straightforward manner so that it is independent of the speckle modulation model, depending only on the statistics of the modulation spectrum. A contract that is now in progress (F30602-76-C-0021) will accomplish this extension. We also expect that modifications to the servo, 


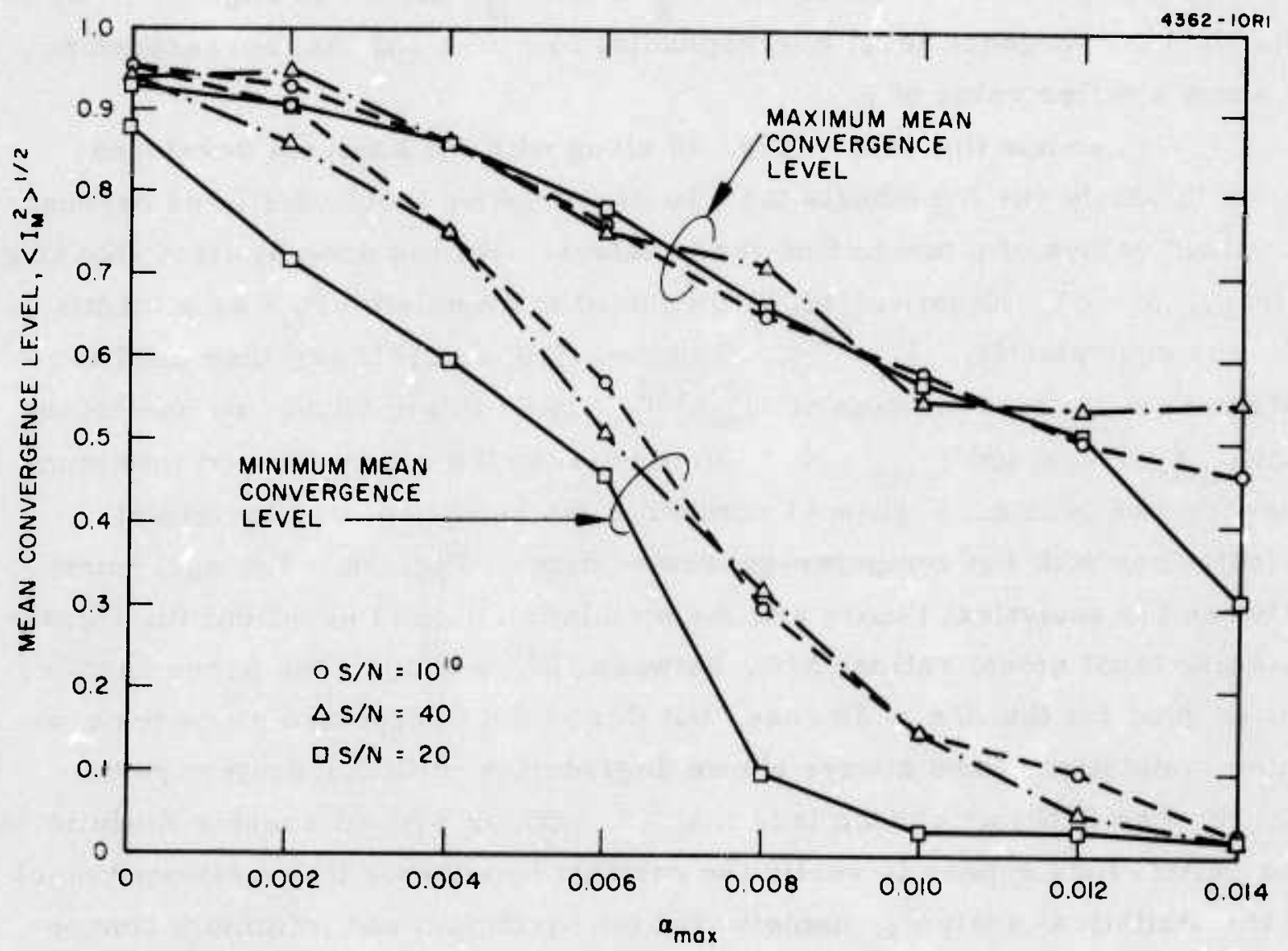

Fig. 40. COAT convergence levels as a function of speckle noise amplitude as observed by computer simulation. 


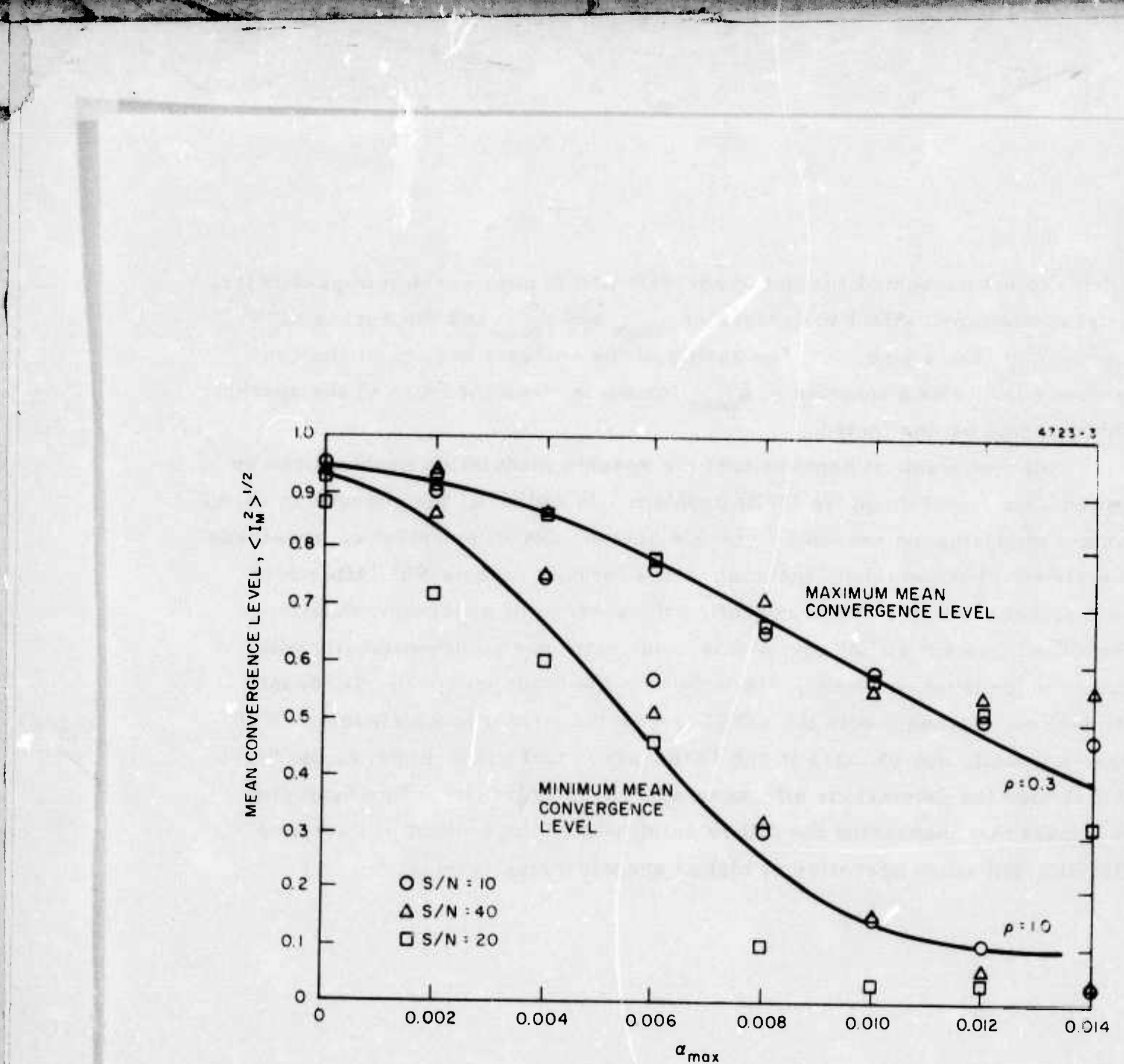

Fig. 41. Convergence level versus $a_{\max }$ theory versus experiment for an 18-element COAT array $\left(\psi=20^{\circ}\right)$ 
which are not accounted for in the analysis (the details of the low-pass filter, for example), will affect the values of $\rho_{\max }$ and $\rho_{\min }$ and the curves $\left\langle I_{m}^{2}\right\rangle^{1 / 2}$ versus $a_{\max }$ for a given $\rho$. The ability of the analysis to predict the convergence levels as a function of $a_{\max }$ (or some other measure of the speckle noise) should be unaffected.

At this point, it appears that the speckle modulation problem can be viewed as a signal-to-noise (S/N) problem. In addition, the changes in COAT system performance caused by speckle "noise" are independent of broadband. The signal-to-noise (shot, thermal, etc.) ratio as long as this latter S/N ratio is sufficient for stable operation the absence of speckle modulations. The COAT system signal-.to-speckle noise ratio varies dramatically with convergence level, however, since the speckle noise within the ditherband competes so strongly with the COAT-generated convergence signals. With this viewpoint, any changes in the COAT servo that act to improve the $S / N$ will reduce the deleterious effects of speckle modulations. For example, we expect that increasing the dither amplitude or the amount of low-pass filtering will allow operation at higher speckle-noise levels. 
V. SUMMARY AND CONCLUSIONS

A. Turbulence and Blooming Compensation

This program has continued to build the experimental capabilities of the DARPA/RADC COAT experimental model and to increase its versatility as a breadbiad for studying multidither COAT concepts and performance. This system has proved to be the single most valuable tool available to date for such studies, rivaled only by the detailed computer simulations that have been developed along with it. The COAT system now has 18 phase control channels, two tracking control channels, and one focus control channel. For propagation studies, there are several the rmal blooming and turbulence generators, including a $100 \mathrm{~m}$ outdoor path at Hughes Research Laboratories.

The DARPA/RADC COAT system has demonstrated that an improvement factor of about 1.5 is possible against blooming distortions, with the compensation occurring only for distortions in the first $30 \%$ of the propagation path. Compensation for turbulence and fixed distortions is not impaired by the presence of the rmal blooming, even though little correction may occur for the blooming itself. This observation has been verified by both experiment and computer simulation. These results, along with those from an earlier program, ${ }^{1-5}$ indicate that the achievement of the high focal-plane irradiance should not be limited by atmospheric propagation, provided that the rmal blooming distortions are minimized and that a suitable COAT system is used.

Computer simulation data indicate that a return-wave COAT system may not be a suitable concept when the rmal blooming is present. Such systems can remove turbulence and fixed distortions just as a multidither system does, but may become unstable and reduce the focal plane ir radiance if the blooming becomes too severe.

In all of the experiments performed to date, the multidithe $r$ tracking and focus (BAT) controls have not increased the average peak beam irradiance significantly over that produced by the phase controls alone. The only exception to this observation occurred with artificial turbulence 
distortions introduced with a phase screen. ${ }^{6}$ With a different artificial phase screen, the BAT controls had no effect. This somewhat unexpected observation may be a result of the characteristics of our artificial turbulence screen since real turbulence should have significant tilt components (see the results in Section IV as well as Ref. 11).

B. Complex Target Effects Caused by Speckle Modulations

Problems with COAT system convergence caused by specklemodulations have not been observed with an extended, scotchlite target used with the 18-channel COAT system. Artificially-generated amplitude modulations have affected the COAT convergence level, however, in both experiments and computer simulations. It remains to be determined whether real targets can produce significant speckle modulations that will affect real multidither COAT systems. An analytical model has been developed that can accurately predict the convergence level and stability of a multidithe $\mathrm{r}$ COAT system once the system is defined and once the statistics (such as the power spectral density) of the spurious modulations are known. Additional work is continuing on this subject, supported by DARPA/RADC Contract F30602-76-C-0021.

\section{Recommendations for Additional Investigations}

The work on this contract has satisfied the original goals of the program and has contributed substantially to the understanding of COAT systems, their capabilities, and their limitations. There are several areas, however, where additional work is needed. First, we need to determine what targets and scenarios can produce speckle modulations that are strong enough to degrade multidither COAT performance. This work will involve c.nalytical, computer simulation, and experimental efiorts and should inciucie some experimental work at $10.6 \mu \mathrm{m}$, or another convenient infrared wavelength. A current contract, F30602-75-C-00321, is aimed at some of this work, but does not include 10.6 in experime: measurements. 
A second area of investigation is in the rmal blooming compensation. All of the work to date, at Hughes as well as at other laboratories, indicates that present COAT techniques will not do much for thermal blooming with cw lasers. Other techniques such as different multidither concepts, irradiance tailoring, wavelength selection, and pulsed operation should be pursued to find those concepts and systems that minimize thermal blooming or increase the ability of COAT to compensate for those blooming distorions that do occur.

There is still much to be done in the a rea of computer simulation. The codes developed to date for both COAT and propagation have been invaluable in identifying and studying propagation phenomena and compensation for them. They have also been essential for designing and evaluating COAT hardware. Additional work is required to improve these codes as well as to utilize them in studying COAT compensation with various system and target scenarios. We need to develop efficient codes that can simulate thermal blooming compensation with multidithe $\mathrm{COAT}$ system and realistic, multiple-glint targets.

Since most of the high-energy laser applications of COAT are at infrared wavelengths, much experimental work needs to be done to demonstrate the required hardware technology, which can be quite different with high-power, infrared lasers than with low-power, visible wavelength devices. The HICLAS program (contract N6092l-76-C-0008 and its follow-on) will address some of these technology questions. In addition, low-power COAT experiments with infrared wavelengths are in order to investigate target effects such as speckle and glint destruction that may be quite different at infrared wavelengths than in the visible.

Firally, as promising new COAT servo and/or system concepts are invented, they should be investigated in low-power, breadboard experiments to establish what advantages they may have in compersation performance, versatility, simplicity in hardware design and implementation, or reliability of operation under a variety of operational conditions. 
RHFlORFNCIES

1. W. 13. Bridges, et al., "Coherent Optical Adaptive Techniques, RADC report No. RADC-TR-73-384, July 1973. (772 639)

2. W. B. Bridges, et al., "Coherent Optical Adaptive Techniques," RADC report No. RADC-TR-74-38, Oct. 1973. (776 814)

3. W. B. Bridges, et al., "Coherent Optical Adaptive Techniques, RADC report No. RADC-TR-74-108, Jan. 1974. (779 668)

4. W. B. Bridges, et al. , "Coherent Optical Adaptive Techniques, RADC report No. RADC-TR-74-187, Apr. 1974. (783 281)

5. J. E. Pearson, et al., "Coherent Optical Aclaptive Techniques," RADC report No. RADC-TR-75-46, J an 1975. (A007 032)

6. J. E. Pearson, et al., "COAT Measurements and Analysis," Quarterly Tech. Report No. 3, Contract F30602-75-C-0001, Nov. 1975.

7. J. E. Pearson, "COAT Measurements and Analysi s," RADC-TR-75-47, Feb. 1975. (A006 105)

8. J. E. Pearson, "COAT' Measurements and Analysis," RADC-TR75-101, May 1975. (A011 707)

9. J. Wallace and M. Camac, J. Opt. Soc. Am. 60, 1587 (1970).

10. F. G. Gebhardt and D. C. Smith, IEEE J. Quantum Electron. QFi-7, $63(1971)$.

11. D. L. Fried, J. Opt. Soc. Am. 55, 1427 (1965).

12. J. E. Pearson, "Atmospheric Turbulence Compensation Using Coherent Optical Adaptive Techniques (COAT), "to be published in Applied Optics.

13. W. P. Brown, Jr. and J. E. Pearson, "Multidither COAT Compensation for Thermal Blooming and Turbulence: Experimental and Computer Simulation Results (U), "presented at the First DOD on High Energy Laser Technology, San Diego, California, 1-3 October 1974.

14. W. B. Bridges and J. E. Pearson, Appl. Phys. Lett. 26, 539 (1975).

15. J. E. Pearson, "Multidither COAT Experimental Results," presented at DARPA/Navy Adaptive Optics Symposium, Lincoln Laboratory, Massachusetts, 3 Dec. 1974. 
16. C. A. Primmerman and D. G. Fouche, J. Opt. Soc. Am. 6,5, $1212(1975)$.

17. F. C. Gebhardt and J. S. Kendall, unpublished.

18. W. P. Brown, J r., unpublished。

19. L. Rradley and J. Herrmann, Appl. Opt. 13, 331 (1974).

20. D. G. Fouche, unpublished.

21. JoWallace, I. Itzkam, and J. Camm, J. Opt. Soc. An. 64, 1123 (1974).

22. C. Yeh, JoE. Pearson, and W. P. Brown, Jr., "Enhanced Target Irradiance in the Presence of Thermal Blooming, " submitted to Appl. Opt.

23. J. E. Pearson, C. Yeh, and W. P. Brown, Jr., "Propagation of a 'Holc-in-the-middle' Beam in the Presence of Thermal Blooming," submitted to J. Opt. Soc. Am.

24. J. N. Hayes, Appl. Opt. 13, 2072 (1974).

25. J.Wallace and J. Q. Lilly, J. Opt. Soc. Am. 64, 165 (1974).

26. T. R. O'Meara, "Classification of Adaptive Arrays," unpublished.

27. J. Herrmann, J. Opt. Soc. Am. 65, 1211 (1975).

28. J. E. Pearson, M. E. Pedinoff, and S. A. Kokorowski, "Recent Advances in Coherent Optical Adaptive Techniques, "presented at the 1975 Conference on Laser Engineering and Applications, Washington, D.C., May 1975.

29. R. F. Ogrodnik and G. Gurski, "Target Return - Adaptive Aperture System Interaction Effects (U), "presented at lst DoD Conf. on High Energy Laser Technology, San Diego, Calif., October 1974.

30. W. P. Brown, Jr., "Computer Simulation of Adaptive Optical Systems," Contract N60921-74-C-0249, Final Tech. Report, Sept. 1975. 

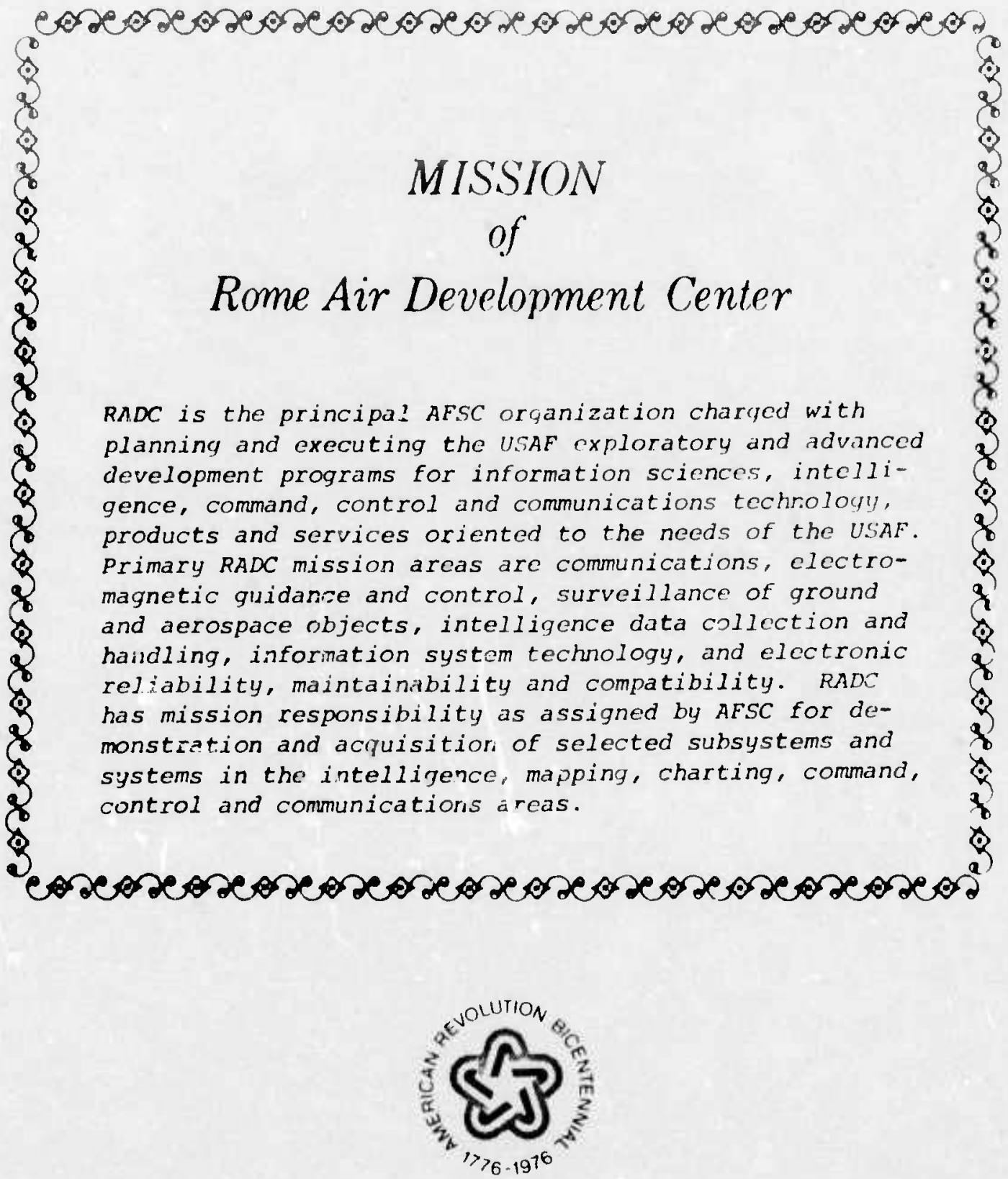